\title{
Genetic factors driving the functional specification of spinal motor neurons
}

\author{
Dissertation \\ In partial fulfilment of the requirements for the degree \\ "Doctor of Philosophy (PhD)" \\ Division of Mathematics and Natural Sciences \\ of the Georg August University Göttingen, \\ Faculty of Biology
}

Submitted by:

Tsung-I Lee

Born in

Changhua, Taiwan 


\section{Members of the Thesis Committee}

Supervisor, PhD committee member: Dr. Till Marquardt

Developmental Neurobiology

European Neuroscience Institute, Göttingen

PhD committee member: Prof. Dr. med. Swen Hülsmann

Department of Neurophysiology

DFG Research Center Molecular Physiology of the Brain, Göttingen

PhD committee member: Prof. Dr. Klaus-Armin Nave

Department of Neurogenetics

Max Planck Institute of Experimental Medicine, Göttingen

Date of the oral examination: 


\section{Affidavit}

Here I declare that my doctoral thesis entitled, "Genetic factors driving the functional specification of spinal motor neurons" has been written independently with no other sources and aids than quoted.

Tsung-I Lee

Göttingen, May 2012 


\section{Table of contents}

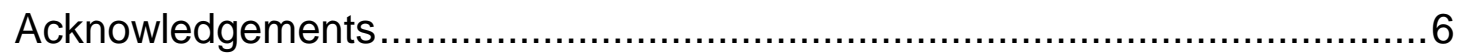

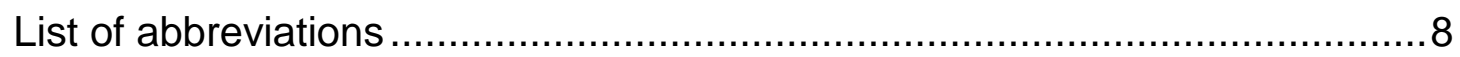

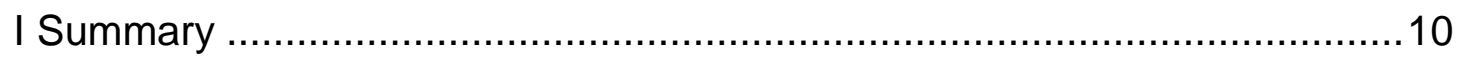

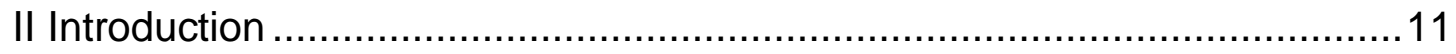

2.1 Spinal motor neurons and the control of movements.......................11

2.2 Development of spinal motor neurons ........................................... 13

2.3 Sensory receptor: muscle spindle ................................................ 18

2.4 Sensory receptor: Golgi tendon organ .......................................20

2.5 The function of gamma-motor neurons .......................................20

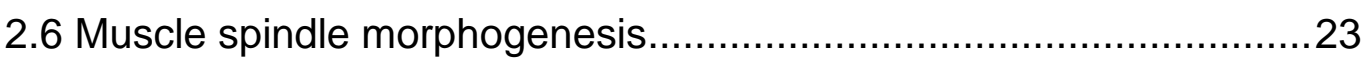

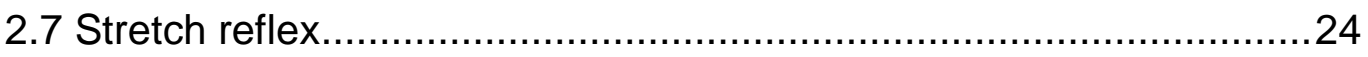

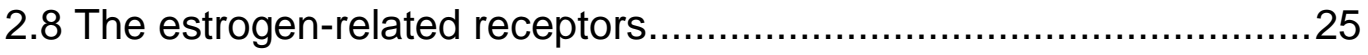

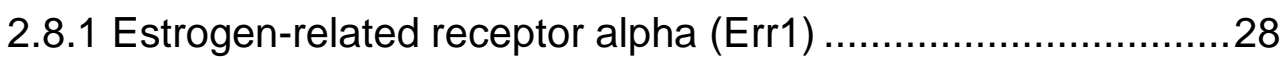

2.8.2 Estrogen-related receptor beta (Err2) …..............................29

2.8.3 Estrogen-related receptor gamma (Err3) ............................29

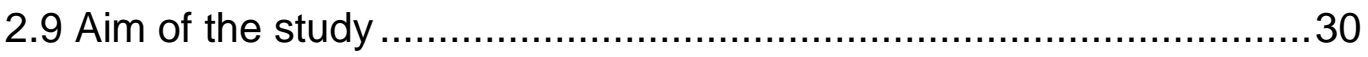

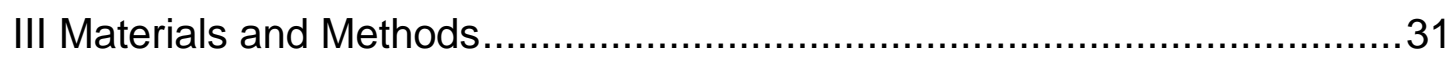

3.1 Laboratory consumables and plastic ware ...................................... 31

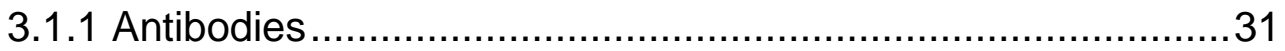

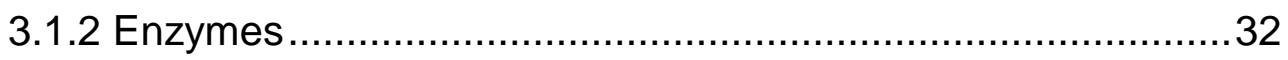

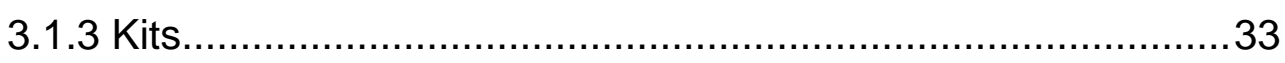

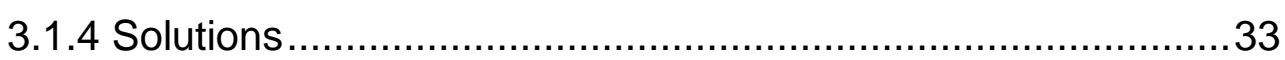

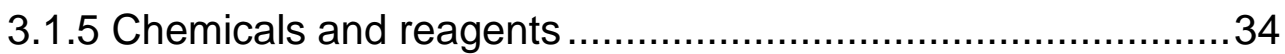

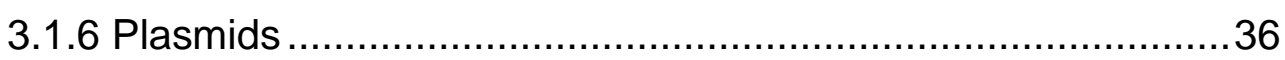

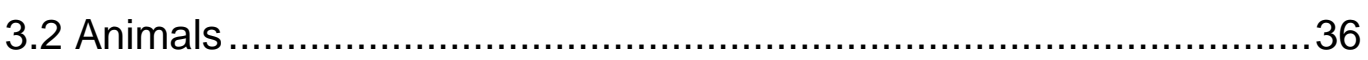

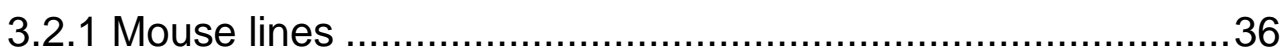

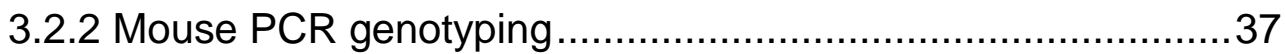

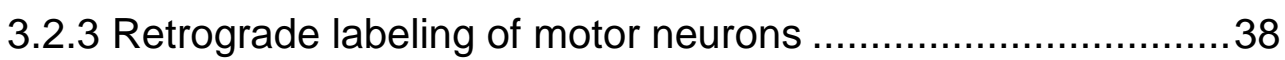

3.2.4 Isolation of retrogradely labeled motor pools ..........................38

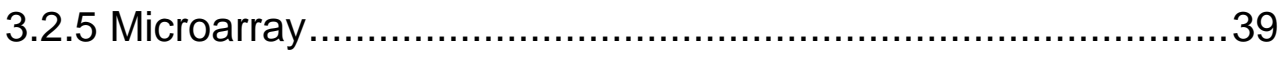

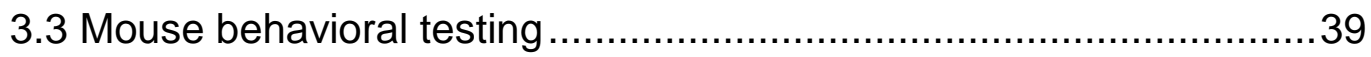

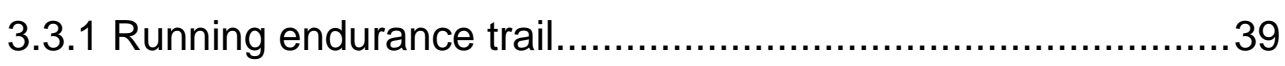

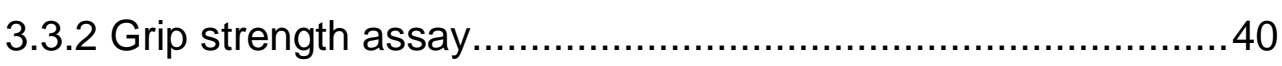

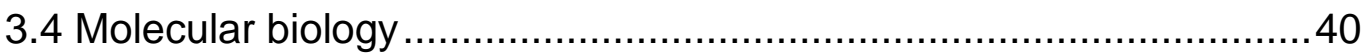

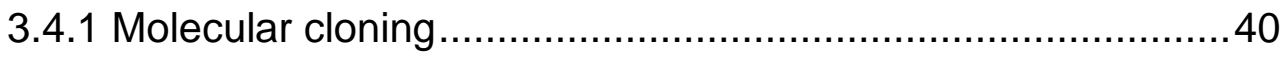

3.4.2 In situ hybridization ......................................................... 41 


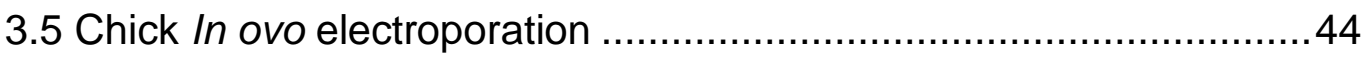

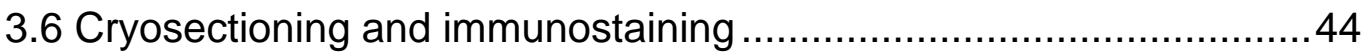

3.7 Tol2 system: long-term expression in chick embryo ........................45

3.8 Motor neuron size distribution histograms ......................................

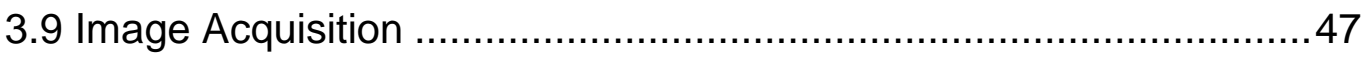

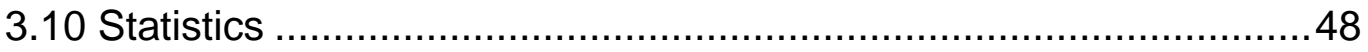

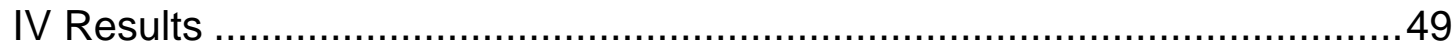

4.1 Expression profiling of functionally different motor neuron pools ...... 49

4.2 Candidate genes in functional motor neuron subtypes .53

4.3 Err2 expression is restricted to a small-sized motor neuron population

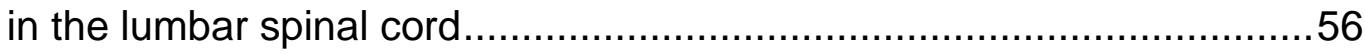

4.4 The functional role of ERR family in spinal motor neurons ...............65

4.5 Stable transgene expression in late gestation chick spinal cord .......77

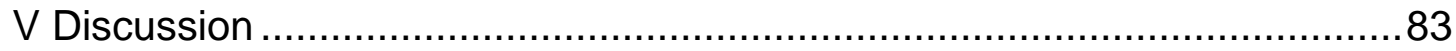

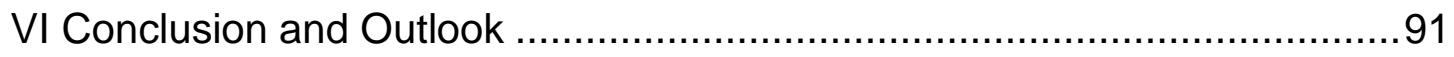

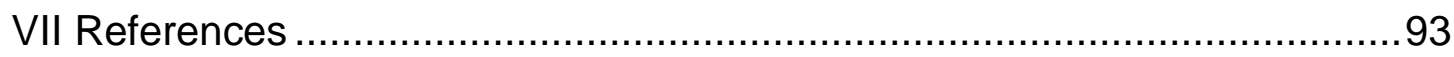

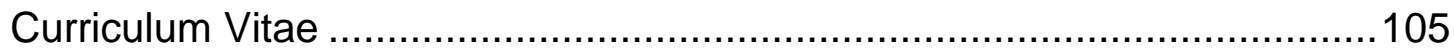




\section{Acknowledgements}

In my four-year PhD period, the sun and the moon move back and forth like a shuttle. First of all, I would like to acknowledge the endless support and insightful opinions from Dr. Till Marquardt who gave me the opportunity to be a PhD student under his supervision.

I also wish to express my gratitude to Prof. Dr. med. Swen Hülsmann and Prof. Dr. Klaus-Armin Nave for sharing invaluable comments during my thesis committee meetings. Additional thanks go to Dr. Lars Wittler at Max Planck Institute of Molecular Genetics Berlin for the help with transcriptome analysis, Dr. Alistair N. Garratt at Max Delbrueck Center for Molecular Medicine Berlin for sharing the Tshz3 antibodies for my project, and Prof. Dr. Klas Kullander at Department of Neuroscience, Uppsala University, Sweden for providing the Err2 and Calca in situ hybridization probes.

I also thank all the former and current members of Dr. Till Marquardt's laboratory: Liang Wang, Daniel A. Müller, David Herholz, Lukas Cyganek, Pitchaiah Cherukuri, Chor Hoon Poh, Camille Lancelin, and Alexandra Klusowski for the great assistance they gave me since I joined the laboratory.

My thanks also go to Heiko Röhse for all the advice in confocal imaging and in the use of necessary computer programs. I am grateful to Anne for being my English language teacher during my thesis preparation.

Pitchaiah deserves special thanks for all the help, patience, and inspiring advice he offered me during my PhD life.

I am also indebted to our beautiful lab technician, Alex for mice genotyping and useful advice about life in general. Many thanks to my friends for always being there for me and for all the happiness and disappointments we had in and out of Göttingen. Special thanks to my parents who have always 
supported my decision and gave me the help more than I expected.

Finally, during these four years, I want to give the biggest thanks to my wife, Hsiao-Chun who always supports my decisions and has enormous patience towards me. Thank you for sharing good and bad times with me and making our life full of happiness. 


\section{List of abbreviations}

$\begin{array}{ll}\text { BMP } & \text { Bone morphogenetic protein } \\ \text { cDNA } & \text { Complementary DNA } \\ \text { Chat } & \text { Choline acetyltransferase } \\ \text { cRNA } & \text { Complementary RNA } \\ \text { CTB } & \text { Cholera toxin subunit B } \\ \text { dl } & \text { Dorsal interneuron } \\ \text { DNA } & \text { Deoxyribonucleic acid } \\ \text { DRG } & \text { Dorsal root ganglion } \\ \text { eGFP } & \text { Enhanced green fluorescent protein }\end{array}$

Egr3 Early growth response 3

EnR Engrailed repressor domain

Er81 Ets variant gene 1

ErbB Epidermal growth factor receptor

ERK Extracellular signal-regulated kinases

Erm Ets variant gene 5

Foxp1 Forkhead box P1

GDNF Glial cell line derived neurotrophic factor

$\mathrm{Hb9} \quad$ Motor neuron and pancreas homeobox 1

Hox Homeobox

IRES Internal ribosome entry site

IsI1 Islet 1 transcription factor

Lhx LIM homeobox protein

mGFP Membrane bound eGFP

MN Motor neuron

mRNA Messenger RNA

NeuN Neuronal nuclei

Nkx6.1 NK6 homeobox 1

NLSeGFP Nuclear localization sequence eGFP

NT-3 Neurotrophin 3

Olig2 Oligodendrocyte transcription factor 2

Pea3 Ets variant gene 4

PGC-1 $\quad$ Peroxisome proliferator-activated receptor gamma

coactivator 1-alpha 


$\begin{array}{ll}\text { RNA } & \text { Ribonucleic acid } \\ \text { Shh } & \text { Sonic hedgehog } \\ \text { TGF- } \beta & \text { Transforming growth factor beta } \\ \text { TrkC } & \text { Neurotrophic tyrosine kinase, receptor, type } 3 \\ \text { Tshz3 } & \text { Teashirt zinc finger homeobox } 3 \\ \text { vGlut1 } & \text { Vesicular glutamate transporter } 1 \\ \text { vAChT } & \text { Vesicular acetylcholine transporter } \\ \text { VP16 } & \text { Herpesvirus protein VP16 } \\ \text { Wnt } & \text { Wingless-related MMTV }\end{array}$




\section{Summary}

Spinal motor neurons which innervate different types of muscle fibers play a pivotal role in controlling body postures and locomotion. Spinal motor neurons can be broadly classified into alpha- and gamma-motor neuron subtypes. Alpha-motor neurons can be further subdivided into fast and slow functional subtypes. It is known that several spatially and temporally orchestrated transcriptional cascades are instrumental in determining and specifying various neuronal subtypes in the neural tube during neurogenesis. However, the factors responsible for determining the specification of functional spinal motor neuron subtypes (alpha versus gamma; slow versus fast) are still unknown. In this study, I performed transcriptome analysis in E18.5 mice to identify the gene expression profiles of functionally distinct motor pools to identify determinants of motor neuron subtype. Through this screen, I identified the orphan nuclear receptor Err2 as a selective marker for gamma-motor neurons. The expression of Err2 paralleled that of the closely related Err3, a previously identified gamma-motor neuron marker. Through novel transposon-mediated gene manipulation in the embryonic chick spinal cord, I found that both Err2 and Err3 are sufficient to promote gamma-motor neuron identity, based on morphological, molecular and initial physiological criteria. Fusion of heterologous transcriptional activation or repression domains further suggested that Err2 and Err3 act as transcriptional activators. My thesis project thereby uncovered first insights into the functional specification of motor neurons, and provided evidence that Err2 and Err3 act as genetically redundant transcription activators that promote the acquisition of gamma-motor neuron identity in subsets of motor neurons. 


\section{Introduction}

\subsection{Spinal motor neurons and the control of movements}

The spinal cord is part of the central nervous system that controls the muscle fibers of limbs and trunk and also receives sensory feedback from these regions in return (Watson et al., 2008). From anatomical evidence, the human body has more than 600 muscles that consist of 0.1 billion muscle fibers. These muscle fibers are innervated by more than 120,000 spinal motor neurons. Basically, each muscle fiber is innervated by single motor neuron, but a single motor neuron can innervate more than a hundred muscle fibers. A muscle is innervated by several motor neurons which are usually clustered into an elongated motor pool in the ventral spinal cord (Romanes, 1941; Romanes, 1964). The motor unit is the basic component for motor control during vertebrate movement. A motor unit consists of a single spinal motor neuron and all the muscle fibers it forms neuromuscular synapses with (McPhedran et al., 1965). Moreover, all muscle fibers which a single motor neuron innervates are of the same type. Motor units have distinct physiological properties and are basically divided into four groups: fast fatigable (FF), fast fatigue-resistant (FR), fast fatigue-intermediate (FI), and slow (S) motor units (Bessou et al., 1963; Burke et al., 1973). In a FF motor unit, a FF motor neuron innervates type IIb (fast fatigable) muscle fibers, which generate the highest force and fastest contraction speed but which fatigue in a few seconds. In FR motor unit, a FR motor neuron innervates type lla (fast fatigue-resistant) muscle fibers, which generate moderately fast contraction speed and intermediate force with the capacity to resist fatigue for a few minutes. FI motor unit has the physiological properties between the FF and FR motor units. Finally, a slow motor neuron 
innervates type I (slow-twitch) muscle fibers, which generate the lowest force and slowest contraction speed, but are highly fatigue resistant (Kernell et al., 1999; Kernell, 2003). In addition, spinal motor neurons can be further divided into functionally diverse classes and subtypes according to the muscle types which each neuronal subtype innervates. (1) The motor nerve innervation of the extrafusal muscle fibers is derived from large soma-sized motor neurons, termed alpha-motor neurons. (2) The small soma-sized motor neurons which the intrafusal muscle fibers of muscle spindles are termed gamma-motor neurons. (3) The beta-motor neurons are thought to innervate both extrafusal and intrafusal muscle fibers, but this motor neuron population is still not well-defined (Kuffler et al., 1951; Burke et al., 1977; Westbury, 1982; Shneider et al., 2009; Manuel and Zytnicki, 2011). There are two major anatomical characteristics that can be used to distinguish alpha- and gamma-motor neurons in the spinal cord. First, the soma of alpha-motor neurons is significantly larger compared to gamma-motor neurons. Second, proprioceptive sensory neurons only make direct glutamatergic (vGlut1-postive) synaptic input with alpha- but not with gamma-motor neurons (Eccles et al., 1960; Friese et al., 2009). Based on previous investigation of spinal motor neurons, various criteria have been used to characterize the subgroups of fast and slow motor neurons, including after-hyperpolarization half decay times (AHP), rheobase, input resistance, soma size (measured by cell capacitance), and axonal conduction velocity (Eccles et al., 1957; Gardiner, 1993). Basically, fast motor neurons have relatively larger soma size, lower input resistance, higher excitation threshold, and shorter AHP half decay times in comparison to slow motor neurons. Both, cell-intrinsic as well as extrinsic programs have been proposed to determine the diversity of motor neuron subgroups and 
selective innervation of distinct muscle fiber types. To date, however, molecular determinants underlying the specification of fast and slow or alphaand gamma-motor neurons remain unknown.

\subsection{Development of spinal motor neurons}

During embryonic development, the central nervous system is polarized along its anterior-posterior and dorsal-ventral axes (Dasen and Jessell, 2009). The spinal cord can be separated into dorsal and ventral regions. The dorsal horn is the region where inputs from sensory neurons enter the spinal cord. Some proprioceptive afferents that provide feedback from muscle and tendon project to lamina IX in the ventral horn of the spinal cord to form direct synaptic contacts with motor neurons or relay interneurons which are located around the center of the spinal cord (Watson et al., 2008). Within the spinal cord, different populations of spinal interneurons conduct electrical information between sensory and motor neurons (Goulding, 2009; Grossmann et al., 2010).

During development, several secreted factors coming from surrounding tissues mediate the polarity of neural tube. In brief, these factors consist of the secreted morphogenic protein Sonic hedgehog (Shh) from notochord and floor plate, as well as fibroblast growth factors (FGFs) and retinoic acid secreted from the paraxial mesoderm, and proteins of the TGF- $\beta$ superfamily secreted from the roof plate (Jessell, 2000; Shirasaki and Pfaff, 2002; Dessaud et al., 2008). These secreted signals impose the patterning of the neural tube by regulating the temporal-spatial expression of specific sets of transcription factors. Defining dorsal interneuron subtypes requires multiple proteins of the TGF- $\beta$ superfamily, including BMP4, BMP7, dorsalin, and activin from the 
epidermal ectoderm (Liem et al., 1995). Several BMPs are expressed in the roof plate and induce neuronal cell types in the dorsal neural tube. Different populations of dorsal interneurons are also exposed to distinct dosage of BMPs in dorsal neural tube during embryogenesis. The cells that are close to the roof plate are exposed to a high concentration of BMPs and differentiate into dl1 interneurons (Wine-Lee et al., 2004). The next five groups of neurons, being exposed to lower concentrations of BMPs, differentiate into dl2 to dl6 interneurons (Liem et al., 1995; Lee and Jessell, 1999).

Shh acts as a long-range graded morphogen to specify the neuronal fates of cells located in the ventral part of the neural tube (Echelard et al., 1993). The release of Shh by the notochord and floor plate results in a Shh concentration gradient across the ventral part of the neural tube. The cells close to the floor plate that are exposed to high concentrations of Shh differentiate into V3 interneurons (Briscoe and Ericson, 1999; Briscoe et al., 1999). The immediately dorsally located neuronal subtype which receives slightly lower Shh concentrations will become motor neurons. The next three groups of neurons, being exposed to gradually lower Shh concentrations, differentiate into V2, V1, and V0 interneurons (Dessaud et al., 2008). It is though that this gradient of Shh activity establishes neuronal identities in the ventral spinal cord by inducing the expression of distinct sets of transcription factors in neural tube progenitor cells (Figure 1A). 


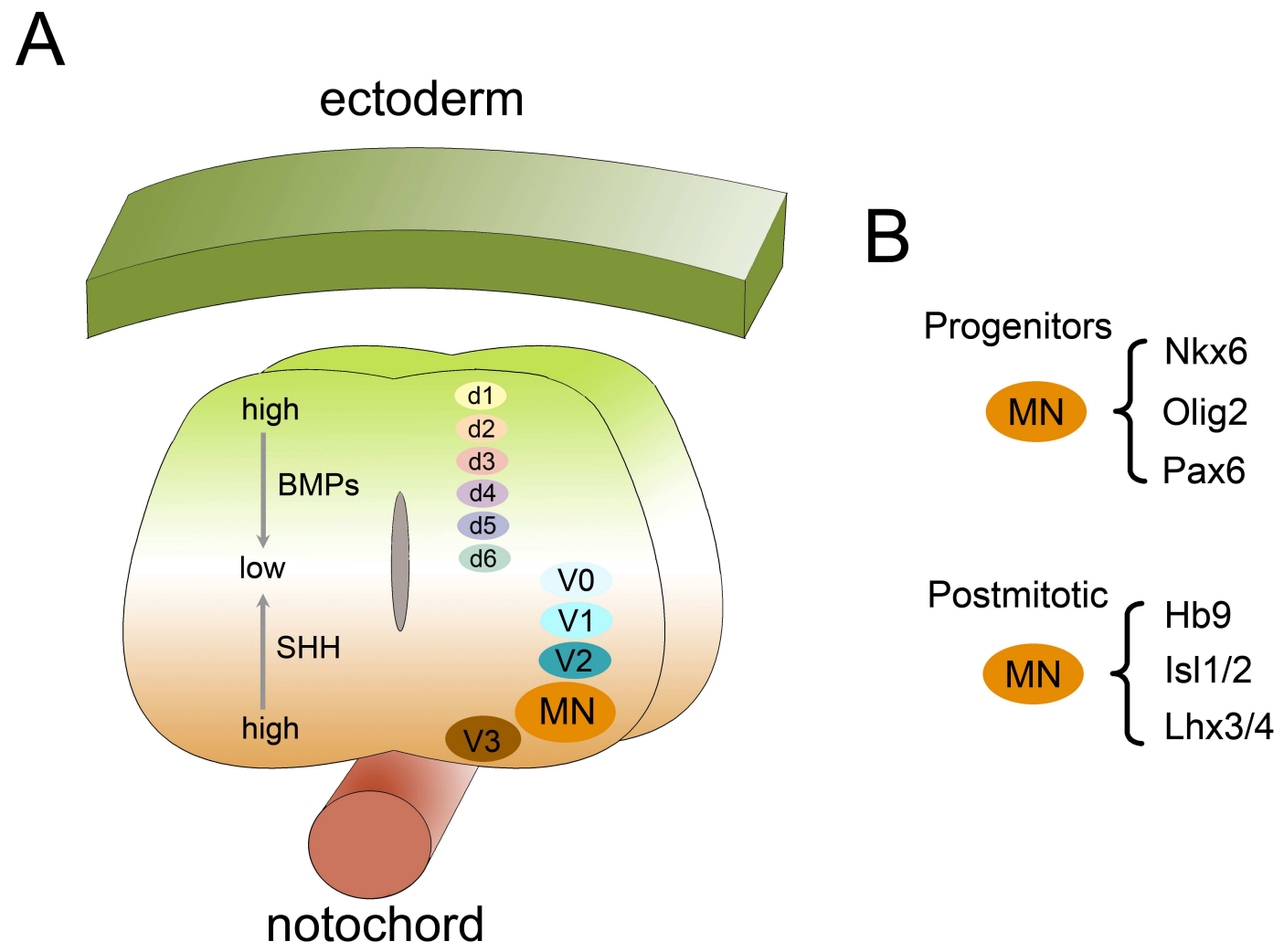

Figure.1 The specification of vertebrate neural tube. (A) Development of the neural tube is mainly regulated by two signaling pathway. BMPs derived from ectoderm modulate the patterning of the dorsal neural tube whereas the ventral neural tube patterning is regulated by Shh secreted from the notochord. Both BMPs and Shh establish gradient signaling to regulate the cell fates of neuronal subtypes in the spinal cord. These secreted proteins induce the different expression of transcription factors expression and thus specify the dorsal-ventral cell lineages. (B) In early developing neural tube, transcription factors like Nkx6, Olig2, and Pax6 are involved in the differentiation of motor neuron progenitors. Subsequently, Hb9, Isl1/2, Lhx3/4 determine the formation of postmitotic motor neurons in E9.5 spinal cord.

Cross-repressive interactions of these transcription factors eventually results in the establishment of discrete progenitor domains in the ventral spinal cord (Dasen and Jessell, 2009). Motor neuron progenitors are established by the combinatorial expression of transcription factors, including the homeodomain and helix-loop-helix proteins Pax6, Nkx6.1, and Olig2 (Novitch 
et al., 2001; Vallstedt et al., 2001; Zhou and Anderson, 2002). Upon cell cycle exit, a unique set of transcription factors ( $\mathrm{Hb}$, Is $\mid 1 / 2$, and $\mathrm{Lh} \times 3 / 4)$ eventually consolidates the cell fate of postmitotic motor neurons (Figure 1B) (Pfaff et al., 1996; Sharma et al., 1998; Arber et al., 1999; Thaler et al., 1999). After the cell fate of motor neurons is determined, it still needs guidance cues to navigate motor neuron axons to their specific targets (Dalla Torre di Sanguinetto et al., 2008). It has been shown that transcription factors of the LIM homeodomain and Hox protein family contribute to define both motor neuron column and motor pool identities (Dasen and Jessell, 2009). Motor neurons responsible for innervating a single muscle are usually organized into a longitudinal pool. These motor pools are in turn clustered within three major columns, according to the targets which each column innervates (Lumsden, 1995). The identity of motor neurons in the medial motor column is defined by Lxh3/4 transcription factors and Wnt4/5 signaling throughout the spinal cord (Agalliu et al., 2009). These motor neurons innervate the axial muscles. In the thoracic spinal cord, Hoxc9 defines motor neurons in the preganglionic column, which project to the sympathetic chain ganglia. In addition, Hoxc9 establishes the hypaxial motor column identity, which innervates the body wall and intercostal muscles (Dasen et al., 2008). Another Hox transcription factor, Hoxc6 is expressed in the lateral motor column of the brachial spinal cord which innervates the musculature of the forelimb (Dasen et al., 2003). Further, Hoxa10, Hoxc10, and Hoxd10 define the lateral motor column in the lumbar spinal cord, which innervates the hindlimb (Figure 2) (Shah et al., 2004; Choe et al., 2006). It is thought that cross-repressive interactions between Hox proteins are important for establishing sharply delineated spinal motor columns. Recently, it has been shown that Foxp1 acts as an accessory factor to Hox proteins that serves to 
regulate the differentiation of the preganglionic column and lateral motor column (Rousso et al., 2008). Inactivation of the Foxp1 gene abolishes preganglionic column and lateral motor column identities, prompting the exclusive generation of axial and hypaxial motor columns throughout the spinal cord (Dasen et al., 2008).

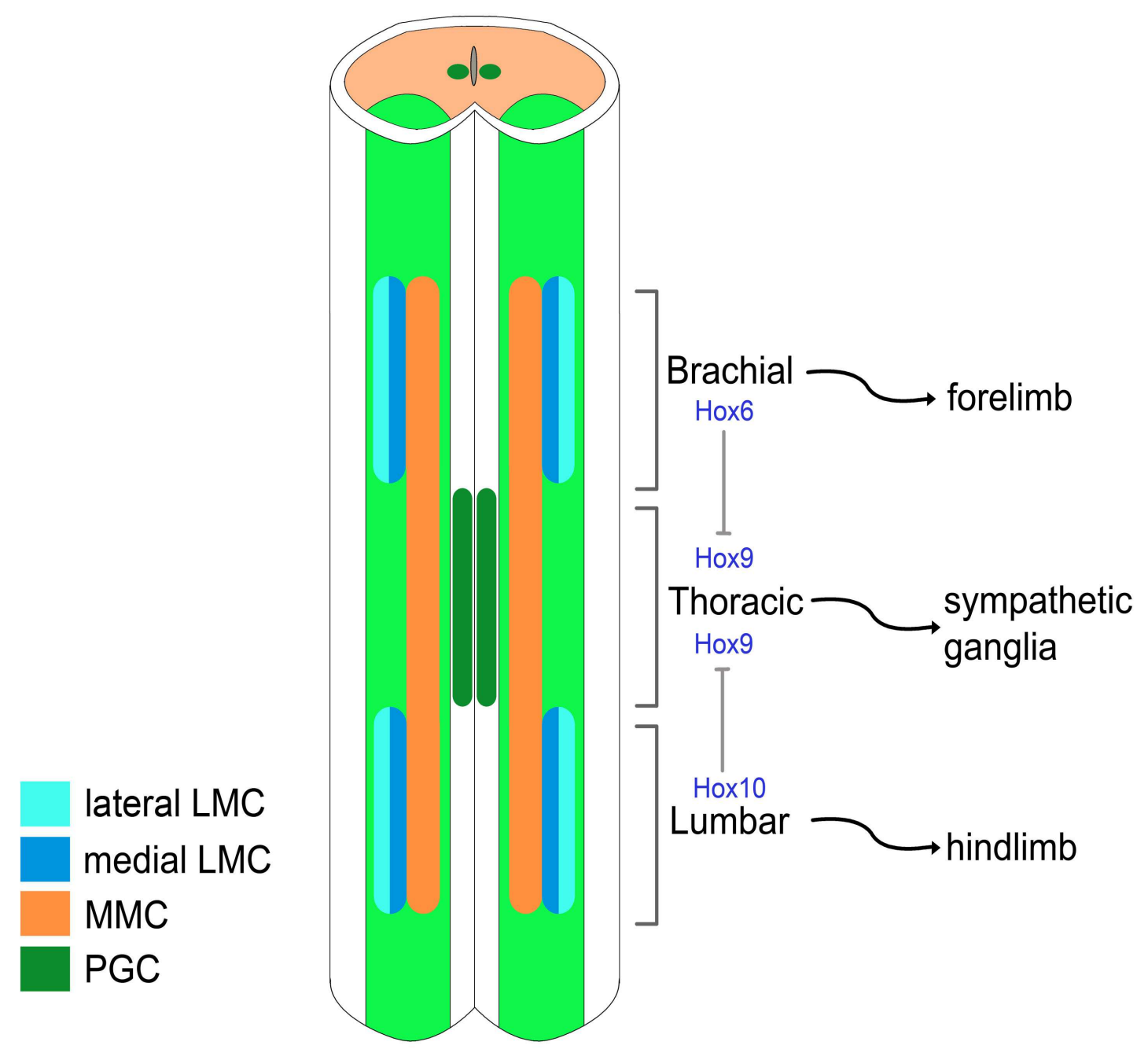

Figure 2. The organization of spinal motor neuron columns. In the spinal cord, Hox transcription factors define distinct motor columns during embryogenesis. The cross-repression interaction between Hox6, Hox9, and Hox10 determines the cell fate of LMC, MMC, and PGC. Motor neurons which are located in later motor column (LMC) at brachial and lumbar regions of the spinal cord innervate forelimb and hindlimb respectively. Pregaglionic column (PGC) derived from the thoracic region of spinal cord project into sympathetic chain ganglia. Medial motor column (MMC) which is located lengthwise throughout the spinal cord has innervates the dorsal axial musculature. 


\subsection{Sensory receptor: muscle spindle}

Muscle spindles (Figure 3) are encapsulated sensory receptors with a fusiform shape that are located within skeletal muscles (Figure 4A and 4B) (Banker and Girvin, 1971). A muscle spindle has three components: (1) 6 or 12 intrafusal fibers whose central regions are noncontractile, (2) myelinated sensory endings and motor endings that innervate the contractile regions of the intrafusal fiber, and (3) connective tissues and extracellular matrix that encapsulate to the structure of the receptor (Coers, 1959; Kandel et al., 2000). The major functions of muscle spindles are to adjust muscle length and to provide feedback to the central nervous system regarding relative positions of body parts during movements. Basically, there are three basic types of intrafusal muscle fibers: (1) nuclear bag fibers, (2) nuclear chain fibers, and (3) dynamic nuclear bag fibers (Ovalle, 1972). These intrafusal muscle fibers can be distinguished according to their shapes and the type of information they relay. When spindle afferents decrease their firing rate, the intrafusal fibers are shortened. Therefore, when a muscle is stretched, the activity in the sensory endings of muscle spindles is increased (Katz, 1950). Muscle spindles are aligned in parallel with the extrafusal muscle fibers that mediate overall contraction of the muscle. According to the innervation pattern seen on intrafusal fibers, there are principally two types of sensory endings: annulospiral endings and flower spray endings (Uehara, 1973). The type la sensory axons (also called primary afferents) spirals around the central area of the muscle spindles. These la sensory endings are very sensitive to acute changes in muscle stretch. Therefore, la fibers and muscle spindles can provide proprioceptive information of rapid or unexpected changes in body or limb positions and generate immediate corrective responses (Mears and Frank, 
1997). Type II sensory axons (also termed secondary afferents) innervate static nuclear fibers and nuclear chain fibers, but not the nuclear bag fibers. The connection between a sensory fiber and an intrafusal fiber is often referred to as a flower spray ending because of the location in the ends (poles) of the axons. When muscles have stopped adjusting their length, type II sensory neurons still keep responding and provide the information of body positions (Lundberg et al., 1987).

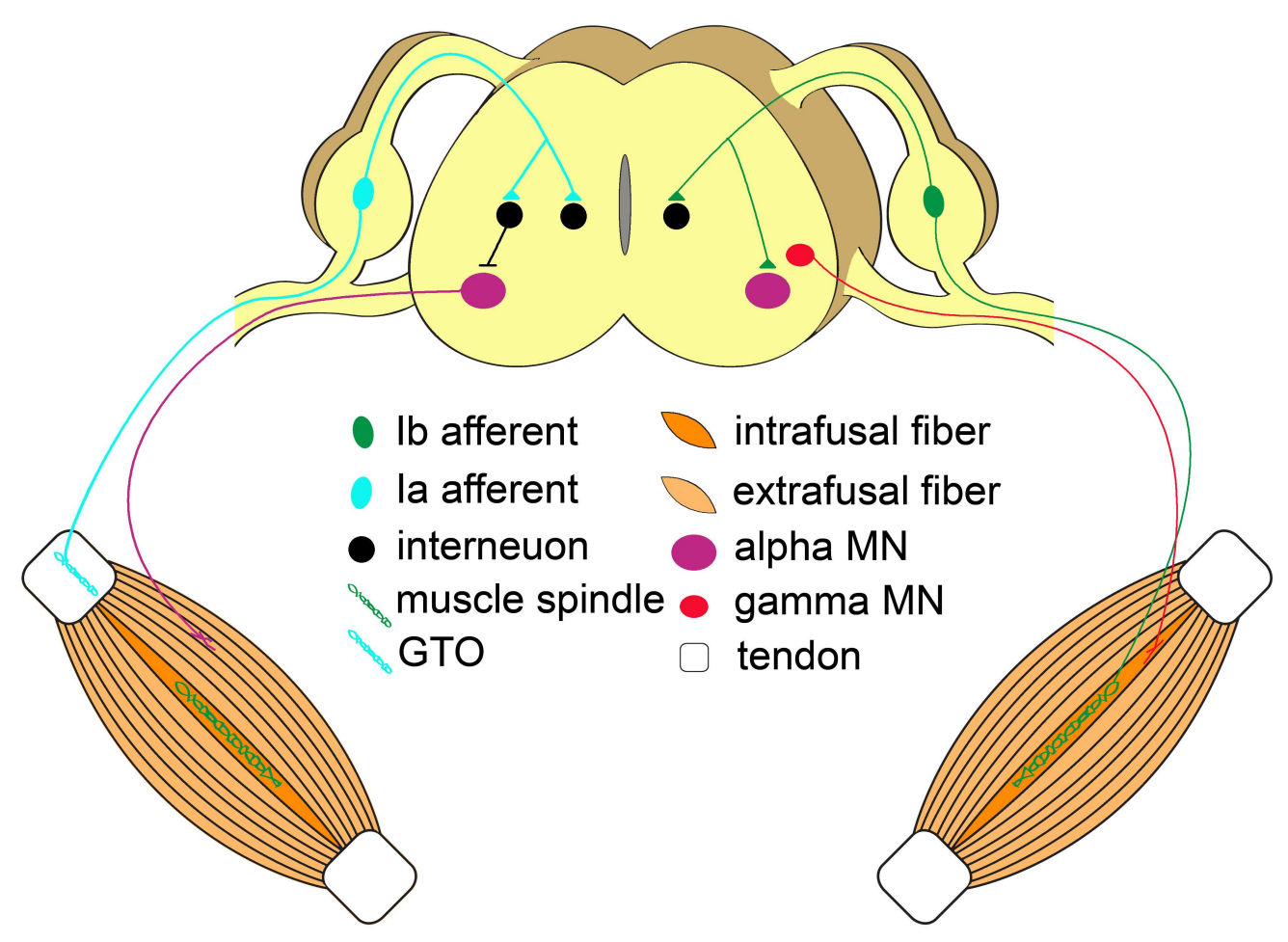

Figure 3. Neuronal innervation and circuitry supplying extrafusal and infrafusal muscle fibers. Right: Type la sensory fibers are also called primary afferent fibers. These sensory fibers have direct synaptic input with alpha-motor neurons (purple) and innervate muscle spindles which are located within intrafusal fibers. Gamma-motor neurons (red) extend their axons to intrafusal fibers and adjust the sensitivity of muscle spindles. Left: Type lb sensory fibers are proprioceptive sensory receptors and locate in the tendons which usually connects muscle to bone. Normally, these sensory fibers have indirect synaptic input with alpha-motor neurons. Both type la and lb fibers synapse with interneurons in the intermediate spinal cord. 


\subsection{Sensory receptor: Golgi tendon organ}

There is another specialized muscle receptor, the Golgi tendon organ (GTO), which is located between muscle fibers and tendons (Figure 3) (Lund et al., 1978; Soukup, 1983). These receptors are composed of several strands of collagen fibers (Figure 4C and 4D). Each GTO is innervated by sensory afferents called type lb fibers which have flower spray-like endings between muscles and tendons (Schoultz and Swett, 1972). When force is generated by the muscles, the GTO stretches and depolarizes the lb sensory axons (Mileusnic and Loeb, 2009). Type lb sensory fibers have the connections with spinal interneurons that also project to the cerebral cortex and cerebellum. The autogenic inhibition reflex is the major spinal reflex associated with $\mathrm{lb}$ fiber activity (Chalmers, 2004). Autogenic inhibition is a protective response that serves to prevent the skeletal muscle from generating force which bones and tendons can not endure. During movements, sensory lb fibers excite spinal motor neurons and affect the timing of transitions between the static and moving phases (Windhorst, 2007). Taken together, muscle spindles conduct the information about the changes in the length of a muscle, whereas GTO conduct information about the force applied to a muscle.

\subsection{The function of gamma-motor neurons}

Gamma-motor neurons synapse with the contractile segments of intrafusal fibers (Manuel and Zytnicki, 2011). The central receptive portion of the intrafusal fibers is simultaneously supplied by type la or type II sensory afferents that dynamically or statically respond to changes in muscle stretch (Botterman and Eldred, 1982). The main role of gamma-motor neurons is to adjust spindle length during muscle contraction, which it achieves by 
controlling the contraction of the intrafusal muscle fibers (Hagbarth et al., 1986). This action is essential for ensuring the sensitivity of type la nerve endings to stretch, which in turn is an important element in the regulation of muscle tone and the adjustment and maintenance of reflexes during movements (Bennett, 1994). This is achieved by coordinated activation of alpha- and gamma-motor neurons during muscle contraction. Maintenance and adjustment of wake muscle tone further depends on descending vestibular and reticular control of gamma-motor neuron activity, determines control over intrafusal fiber contractile status ,which in turn drives alpha-motor neuron activity levels via mono or polysynaptic feedback from spindle afferents (Kandel et al., 2000). Thus, gamma-motor neurons are of paramount importance for neuromuscular function. 

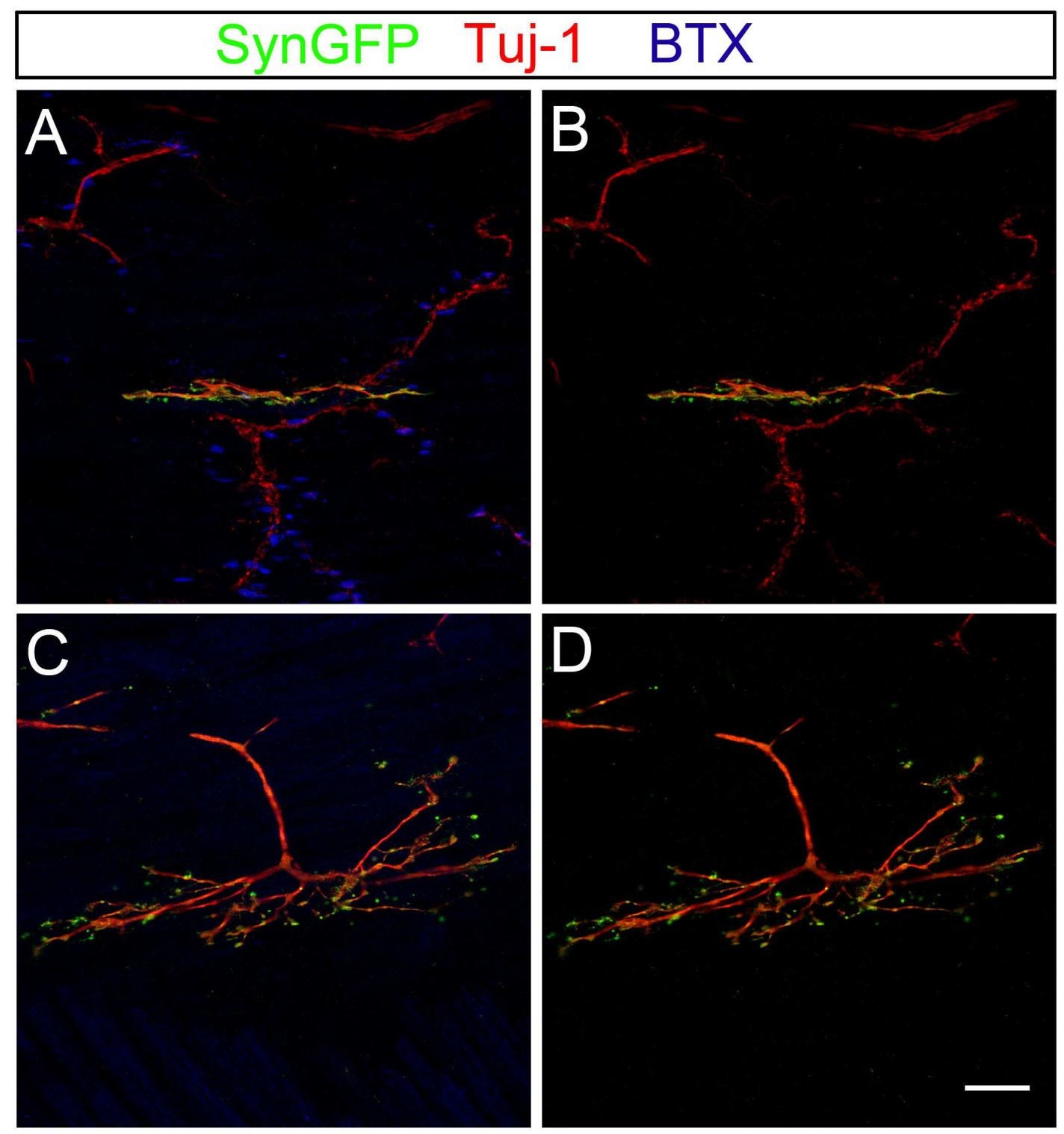

Figure 4. Muscle spindle and Golgi tendon organ in chick hindlimb musclature. Crest3::synatophysin-eGFP vector was electroporated into E2 chick neural tube. In E12 hindlimb tissue, (A and B) Muscle spindle: the type la annulospiral endings of sensory fibers were labeled by SynGFP. (C and D) GTO: the type lb free nerve endings of sensory fibers were labeled by SynGFP. Scale bar: $30 \mu \mathrm{m}$. 


\subsection{Muscle spindle morphogenesis}

During embryonic development, proprioceptive innervation provides signals that induce the differentiation of muscle fibers into functional muscle spindles (Zelena, 1976). At approximately E14.5 in mice, sensory neurons begin to innervate the target muscles; however, they do not have proper connections with type I myotubes yet. The myotubes in embryonic limbs are initially defined by expression of the embryonic slow myosin heavy chain isoform (Tourtellotte et al., 2001). After la sensory innervation, sensory endings provide signals that drive the differentiation of type I myotubes into muscle spindles. Some of type I myotubes which do not receive la sensory innervation will transform to type I extrafusal muscle fibers. It has been shown that several transcription factors, including Egr3, Er81, Erm, and Pea3, are highly expressed in developing muscle spindles after sensory axon innervation and that these factors are required for the normal muscle spindle differentiation (Lin et al., 1998; Arber et al., 2000; Hippenmeyer et al., 2007). The glycoprotein Neuregulin1 released by sensory terminals interacts with the ErbB receptor tyrosine kinase in myotubes, which, in turn increases expression levels of Egr3, Pea3, and Erm in future muscle spindles (Hippenmeyer et al., 2002). In Neuregulin1 conditional knockout mice, the animals failed to induce muscle spindle-specific expression of transcription factors and displayed a loss of annulospiral terminals. Conversely, factors derived from muscle spindles also have a pivotal role in influencing sensory and motor innervation during muscle spindle formation. NT-3 is a muscle spindle-derived neurotrophin that is known to regulate the specification and survival of proprioceptive sensory neurons (Oakley et al., 1997; Genc et al., 2004). For example, removal of chick embryonic limb buds can promote the 
cell death of TrkC expressing sensory neurons in the DRG, indicating that neurotrophic factors derived from skeletal muscles are also important for the survival of proprioceptive sensory neurons (Oakley et al., 1997).

\subsection{Stretch reflex}

The classical monosynaptic stretch reflex circuit is the simplest and best-characterized neuronal circuit in the vertebrate central nervous system (Clarac et al., 2000; Chen et al., 2003). Spinal reflexes play a pivotal role in the motor system in the coordination of skeletal muscles during complex body movements. Adaptive motor control requires the perception of body position, which is also termed proprioception (Herrick, 1947; Smetanin et al., 1982). In order to adjust the body to a certain position, it is necessary to receive feedback about the original starting position of musculatures in the limbs, as well as any force generated from extrafusal muscle fibers. Muscle spindles and GTO besides being involved in spinal reflexes, are also responsible for providing sensory information to the motor system about the current situation of muscles and limbs (Wada and Shikaki, 1999). A simple example where adaptive motor control comes in useful is when you are carrying a box and someone unexpectedly places books on your box. Because the muscles in your hands were not prepared to support the additional force, the box should in principle drop. However, a spinal reflex is activated which enables you to stably hold on to the box. The unexpected weight imposed stretches the muscles of the arms, which results in the activation of la afferents from muscle spindles. The type la sensory neurons have their cell bodies in the dorsal root ganglia and they make direct synaptic contacts with alpha-motor neurons in the spinal cord. Thereby, the activation of la afferents induces the 
monosynaptic activation of alpha-motor neurons, which induces a counterpoised contraction of the arm muscles (Lund and Pompeiano, 1968).

\subsection{The estrogen-related receptors}

Members of the nuclear receptor protein family have the ability to directly bind to genomic DNA and regulate the expression of specific genes which control cellular metabolism, homeostasis, and tissue development (Mangelsdorf et al., 1995; Novac and Heinzel, 2004). Hence, these receptors are collectively classified as transcription factors. The members in the nuclear receptor family can be divided into two subgroups: ligand-dependent and ligand-independent (Giguere, 1999). Ligand-dependent nuclear receptors, such as retinoic acid receptor and liver $\mathrm{X}$ receptor-like can control distinct physiological and cellular differentiation programs. So far, most of the nuclear receptors have no well-defined biological ligands and are therefore classified as ligand-independent or orphan nuclear receptors (ONRs). The estrogen-related receptors (ERR), known as $N R 3 B$ family, were the first orphan nuclear receptors to be characterized twenty years ago (Giguere et al., 1988). The ERR family consists of three gene members referred to as Err1 (ERR alpha, Esrra, and Nr3b1), Err2 (ERR beta, Esrrb, and Nr3b2), and Err3 (ERR gamma, Esrrg, and Nr3b3) (Tremblay and Giguere, 2007). The mouse Err1 gene, located on chromosome $19(\mathrm{~A} ; 193.0 \mathrm{cM})$, is translated into a protein of 422 amino acids (Giguere et al., 1988). The mouse Err2 gene, located on chromosome 12 (D2; $1241.0 \mathrm{cM})$, is transcribed into two alternative splicing variants which encode Err2 isoform 1 (454 amino acids) and Err2 isoform 2 (438 amino acid) (Sladek et al., 1997). The mouse Err3 gene, located on chromosome $1(\mathrm{H} 6 ; 1)$, has two transcript variants which encode 
Err3 isoform 1 (458 amino acids) and Err3 isoform 2 (435 amino acid) (Eudy et al., 1998). With few exceptions, most nuclear receptors share a phylogenetically conserved protein domain structure in mouse and chick (Figure $5 \mathrm{~A}$ and $5 \mathrm{~B}$ ). Each member of ERR family proteins has four core domains, DNA binding domain (DBD), ligand binding domain (LBD), modulator domain, and a hinge region (Mangelsdorf et al., 1995). Using unbiased binding site screens, the first ERR-responsive gene characterized the ERR response element as the consensus DNA sequence TCAAGGTCA (Dufour et al., 2007). Each member of ERR family proteins can interact with DNA response element as monomers, homodimers, and heterodimers (Tremblay and Giguere, 2007). In mouse ERR family proteins, each member shares significantly similarity and identity in their DBD and LBD. For example, the DBD of Err2 has $94.8 \%$ identity and $99 \%$ similarity to the DBD of Err3. The LBD of Err2 is $74.1 \%$ identical and $89.1 \%$ similarity to the corresponding domain in Err3. The ERR proteins also have highly conserved protein structures between mouse and chick (Figure 5C). 


\section{A}

mouse

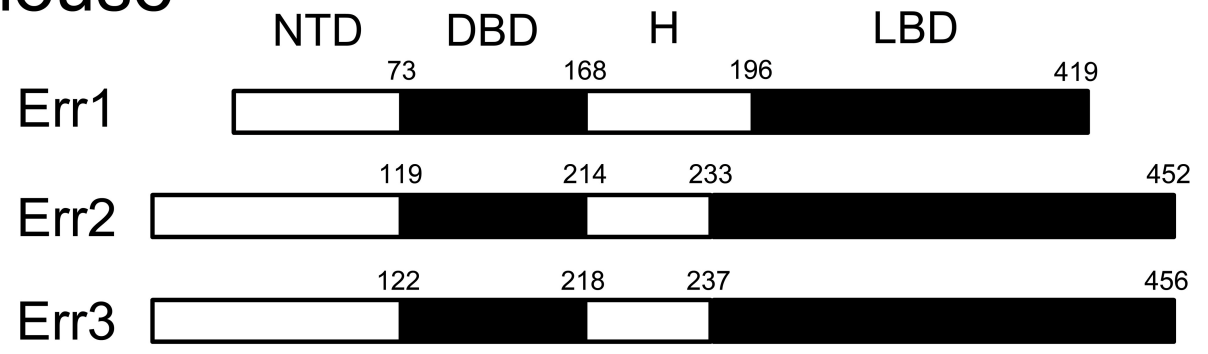

B

chick

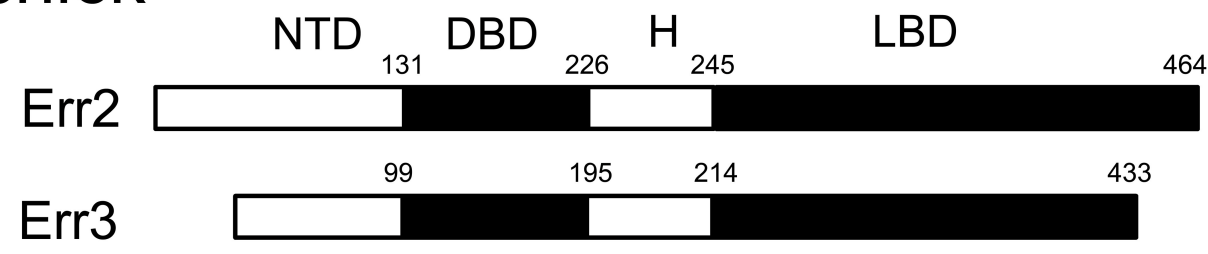

C

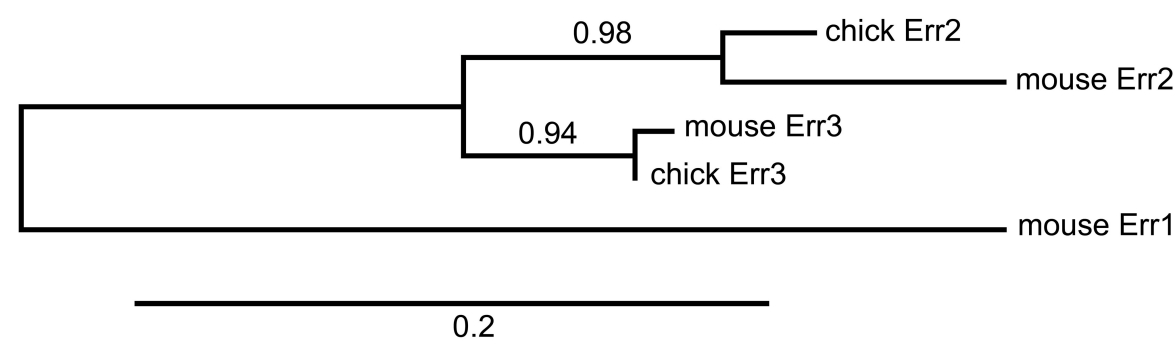

Figure 5. Schematic structure of the different ERR isoforms in mouse and chick. In most orphan nuclear receptors, these proteins have three major domains, a amino-terminal domain (NTD), a DNA binding domain (DBD), and a ligand binding domain (LBD). (A) In mouse species, the ERR family has three nuclear receptor referred to as Err1, Err2, and Err3. (B) Members of the chick ERR family protein include Err2 and Err3. (C) A phylogenetic tree of the ERR family demonstrates that the evolutionary relationships of these orphan nuclear receptors between mouse and chick are closely related to each other. The comparison of ERR proteins in mouse and chick was generated from online analysis programs-Phylogeny.fr. 


\subsubsection{Estrogen-related receptor alpha (Err1)}

During murine organogenesis, Err1 has a tissue wide expression pattern. Err1 mRNA can be detected in the heart, skeletal muscle, osteoblasts, kidney, and cerebellum (Tremblay and Giguere, 2007). In postnatal mice, Err1 is most highly expressed in organs which consume fatty acids as energy sources, suggesting that Err1 plays a prominent role in cellular metabolism. During mouse embryogenesis, Err2 transcripts are first detected in extra-embryonic ectoderm starting at E5.5 (Pettersson et al., 1996). Unlike Err1, the expression of Err2 is more restricted in spinal cord, chorion, and retina. Mouse Err3 expression is abundantly present in the heart and spinal cord at E10.5 (Alaynick et al., 2007). During adulthood, Err3 expression also extends to skeletal muscle, brain, kidney, and spinal cord. Several studies indicate that Err1 is the key regulator of bioenergetic and cellular metabolism. In Err1 knockout mice, null mice have normal fertility with no anatomical alterations, but less body weight and fat deposition in peripheral tissues (Luo et al., 2003). Knocking out Err1 also has a profound effect on myogenesis causing in immature fusion of myotubes and mitochondria dysfunction via aberrant ERK activation. During adipogenesis, Err1 expression is up-regulated in adipose tissue. Under fasting and hypothermia conditions, Err1 can increase the generation of energy in brown fat, liver, and skeletal muscle. Furthermore, PGC-1 $\alpha$ which plays an important role in the control of energy metabolism and mitochondria biogenesis also has been identified as a coactivator of Err1, reinforcing the physiological function of Err1. 


\subsubsection{Estrogen-related receptor beta (Err2)}

Mice embryos homozygous for mutant allele in Err2 gene displayed embryonic lethality by approximately E9.5, resulting in from abnormal chorion development and placental defects (Luo et al., 1997). Although Err2 is essential for chorion formation, conditional knockout mice revealed that mutant embryos can develop normally and produce both genders of fertile adult mice. Recently, it has been shown that Err2 coordinates different genes to regulate the functions and survival of rod photoreceptors (Onishi et al., 2010). Conditional disruption of Err2 causes the rod photoreceptors to degenerate; however this can be rescued by overexpression of Err2. This study provided a drug target of certain retinal dystrophies. In addition, Err2 is one of the transcriptional modulators of ES cell self-renewal and overexpression of Err2 reprograms mouse fibroblasts into pluripotent stem cells Err2 and Nanog were found to cooperate in the regulation of ES cell pluripotency via a network of transcription factors (Zhang et al., 2008). Taken together, these studies revealed that multiple functional roles of Err2 in the control of specific cell types during organogenesis.

\subsubsection{Estrogen-related receptor gamma (Err3)}

Err3 is highly expressed in the fetal heart and spinal cord. Homozygous null mutation of Err3 results in lethality during the first postnatal week due to the lactatemia and electrocardiographic abnormalities (Alaynick et al., 2007). Chip-on-chip analysis demonstrated that Err3 is a key regulator of mitochondrial genetic networks, controlling oxidative metabolic function in heart. Based on clinical studies, Err3 has the potential ability to suppress tumor growth and cell proliferation in prostate cancer cells by arresting 
cell-cycle at G1-S transition (Yu et al., 2007). Recently, Err3 has been identified as a marker of gamma-motor neurons. The gamma-motor neurons were also shown to express low levels of NeuN and high levels of Err3 and during the first 2 weeks of postnatal development. Alpha-motor neurons, in contrast, express low levels of Err3 and high levels of NeuN (Friese et al., 2009). However, it remains unknown whether Err3 plays a role in the specification of gamma-motor neurons or gamma-motor neuron functional properties.

\subsection{Aim of the study}

During embryonic development, a combination of extrinsic secreted factor and cell-autonomous transcription factor networks regulate the specification of spinal motor neuron identities required for establishing topographically correct connections with distinct muscle targets. However, mechanisms underlying the acquisition of inherent functional properties by motor neurons, such as the specification of fast or slow, as well as alpha- or gamma-motor neurons remain elusive.

The aims of this project are:

(A) To identify molecular determinants of motor neuron functional specification using transcriptome analysis.

(B) To establish assays that facilitate investigating the contribution of identified candidate gene activities to motor neuron functional specification in vivo.

(C) To characterize the contribution of identified factors to the functional specification of motor neurons. 


\section{Materials and Methods}

\subsection{Laboratory consumables and plastic ware}

Consumables were purchased from Starlab GmBH (Ahrensburg), Eppendorf (Hamburg), and Sarstedt AG (Nürnstedt). Dissection instruments were bought from Fine Science Tools GmBH.

\subsubsection{Antibodies}

\section{Primary antibodies}

\begin{tabular}{cccl}
\hline Antibody name & Host species & Working dilution & \multicolumn{1}{c}{ Supplier } \\
\hline vAChT & Rabbit & $1: 2000$ & Synaptic Systems GmbH \\
IsI1/2 & Rabbit & $1: 2500$ & Gift from S.L. Pfaff \\
NeuN & Mouse & $1: 1000$ & Millipore AG \\
Err2 & Mouse & $1: 1000$ & R\&D Systems GmbH \\
GFP & Rabbit & $1: 1000$ & Invitrogen GmbH \\
GFP & Sheep & $1: 2000$ & AbD Serotec \\
V5 & Mouse & $1: 1000$ & Invitrogen GmbH \\
Tshz3 & Rabbit & $1: 5000$ & Gift from A.N. Garratt \\
Laminin & Rabbit & $1: 500$ & Sigma Aldrich AG GmBH \\
Tuj-1 & Mouse & $1: 2000$ & Covance GmbH \\
$\begin{array}{c}\text { Alexa Fluor } \\
\text { 647-conjugated } \\
\text { bungarotoxin }\end{array}$ & $\begin{array}{c}\text { Bungarus } \\
\text { multi cinctus }\end{array}$ & $1: 1000$ & Invitrogen GmbH \\
MF20 & Mouse & $1: 200$ & DSHB \\
F59 & Mouse & $1: 200$ & DSHB \\
Is11/2 & Mouse & $1: 200$ & DSHB \\
\hline
\end{tabular}

*DSHB: Developmental Studies Hybridoma Bank 
Secondary antibodies

\begin{tabular}{|c|c|c|c|c|}
\hline $\begin{array}{c}\text { Target } \\
\text { species }\end{array}$ & Host & Conjugate & $\begin{array}{l}\text { Working } \\
\text { dilution }\end{array}$ & Supplier \\
\hline Mouse & Donkey & $\begin{array}{l}\text { Alexa Fluor } 488 \\
\text { Alexa Fluor } 555 \\
\text { Alexa Fluor } 647\end{array}$ & $1: 1000$ & Invitrogen $\mathrm{GmBH}$ \\
\hline Rabbit & Donkey & $\begin{array}{l}\text { Alexa Fluor } 488 \\
\text { Alexa Fluor } 555 \\
\text { Alexa Fluor } 647\end{array}$ & $1: 1000$ & Invitrogen $\mathrm{GmBH}$ \\
\hline $\begin{array}{c}\text { Sheep } \\
\text { Mouse } \\
\text { (IgG1 } \\
\text { subtype) }\end{array}$ & Donkey & Alexa Fluor 488 & $1: 1000$ & $\begin{array}{l}\text { Invitrogen GmBH } \\
\text { Jackson } \\
\text { ImmunoResearch } \\
\text { Laboratories }\end{array}$ \\
\hline
\end{tabular}

\subsubsection{Enzymes}

\begin{tabular}{|c|c|}
\hline Enzyme & Supplier \\
\hline DreamTaq $^{\mathrm{TM}}$ Green Taq DNA polymerase & Fermentas $\mathrm{GmBH}$ \\
\hline Phusion High Fidelity DNA polymerase & New England Biolabs GmBH \\
\hline Restriction enzymes & Fermentas $\mathrm{GmBH}$ \\
\hline Shrimp alkaline phosphatase & Fermentas $\mathrm{GmBH}$ \\
\hline Protein Nucleotide Kinase & Fermentas $\mathrm{GmBH}$ \\
\hline SP6 RNA polymerase & Roche Diagnostics GmBH \\
\hline T7 RNA polymerase & Roche Diagnostics GmBH \\
\hline DNA Ligation Kit & Takara Bio Inc \\
\hline
\end{tabular}




\subsubsection{Kits}

\begin{tabular}{ll}
\hline Kit & Supplier \\
\hline PrimeScript $1^{\text {st }}$ strand cDNA synthesis Kit & Takara Bio Inc. \\
iScript $^{\text {TM }}$ cDNA Synthesis Kit & Bio-Rad GmbH \\
MouseRef-8 v2.0 Expression BeadChip Kit & Illumina, San Diego \\
TotalPrep RNA Amplification Kit & Illumina Inc. \\
QIAfilter Plasmid Maxi Kit & Qiagen GmBH \\
Qiaprep Spin Miniprep Kit & Qiagen GmBH \\
Qiaquick gel extraction Kit & Qiagen GmBH \\
Rneasy micro Kit & Qiagen GmBH \\
TOPO TA Cloning Kit & Invitrogen GmBH \\
\hline
\end{tabular}

\subsubsection{Solutions}

\begin{tabular}{|c|c|}
\hline Solution & Reagents \\
\hline PBS & $\begin{array}{l}\mathrm{pH} 7.2,137 \mathrm{mM} \mathrm{NaCl}, 2.7 \mathrm{mM} \mathrm{KCl}, 10 \mathrm{mM} \\
\mathrm{Na}_{2} \mathrm{HPO}_{4}, 2 \mathrm{mM} \mathrm{KH}_{2} \mathrm{PO}_{4}\end{array}$ \\
\hline PBST & PBS pH 7.2, 0.1\% Tween-20 \\
\hline $\begin{array}{l}\text { Antibody staining } \\
\text { solution }\end{array}$ & PBS pH 7.2, 1\% BSA, 1\% Triton X-100 \\
\hline In situ Blocking buffer & Roche blocking reagent $2 \%(w / v)$ in PBST \\
\hline DNA loading buffer & $8 \%$ Sucrose in TAE, $1 \mathrm{mg} / \mathrm{ml}$ Orange $\mathrm{G}$ \\
\hline TAE buffer $50 x$ stock & $2 \mathrm{M}$ Tris-Acetate, $50 \mathrm{mM}$ EDTA pH 8.0, $57.1 \mathrm{ml}$ \\
\hline solution, $1000 \mathrm{ml}$ & Glacial acetic acid; Add $\mathrm{H}_{2} \mathrm{O}$ up to $1000 \mathrm{ml}$ \\
\hline In situ hybridization & $50 \%$ Formamide, 5x SSC pH4.5, 1\% SDS, 10 \\
\hline buffer & $\mathrm{mg} / \mathrm{ml}$ Heparin, $10 \mathrm{mg} / \mathrm{ml}$ salmon sperm DNA \\
\hline
\end{tabular}


SSC 20x stock solution $3 \mathrm{M} \mathrm{NaCl}, 300 \mathrm{mM}$ sodium citrate $\mathrm{pH} 4.5$

In situ wash Buffer I $\quad 50 \%$ Formamide, $5 x$ SSC pH 4.5

In situ wash Buffer II $\quad 50 \%$ Formamide, 2x SSC pH 4.5

In situ wash Buffer III $\quad 50 \%$ Formamide, $1 \times$ SSC pH 4.5

LB agar

$1 \%(\mathrm{w} / \mathrm{v})$ agar in LB medium

LB medium

$5 \mathrm{~g} / \mathrm{l} \mathrm{NaCl}, 5 \mathrm{~g} / \mathrm{l}$ yeast extract, $10 \mathrm{~g} / \mathrm{l}$ tryptone

MBST

$0.1 \mathrm{M}$ Maleic acid; $0.15 \mathrm{M} \mathrm{NaCl} \mathrm{pH} 7.5,0.1 \%$

Tween-20

$100 \mathrm{mM} \mathrm{NaCl}, 10 \mathrm{mM}$ Tris- $\mathrm{HCl} \mathrm{pH}$ 9.5, $50 \mathrm{mM}$

NTMT

$\mathrm{MgCl}_{2}, 0.1 \%$ Tween-20

Tissue digest buffer

$0.1 \mathrm{M}$ Tris $\mathrm{pH} 8,30 \mathrm{mM} \mathrm{NaCl}, 0.2 \mathrm{mM}$ EDTA,

$0.05 \%$ SDS, $0.5 \mathrm{mg} / \mathrm{ml}$ Proteinase $\mathrm{K}$

\subsubsection{Chemicals and reagents}

\begin{tabular}{ll}
\hline Name & Supplier \\
\hline Agar-Agar & Carl Roth GmbH \\
Agarose NEEO & Carl Roth GmbH \\
Bovine serum albumin (BSA) & Carl Roth GmbH \\
Cholera toxin subunit B conjugates & Invitrogen GmbH \\
DIG RNA labelling mix & Roche Diagnostics GmbH \\
DNA Ladder & Fermentas GmbH \\
dNTP mix & Fermentas $\mathrm{GmbH}$ \\
Diethylpyrocarbonate (DEPC) & Carl Roth $\mathrm{GmbH}$ \\
Ethanol 99.9\% & Carl Roth $\mathrm{GmbH}$ \\
Ethidium bromide & Carl Roth $\mathrm{GmbH}$
\end{tabular}


Ethylenediaminetetraacetic acid

Formamide

GeneRuler 1 kb DNA ladder

Glycerol

Glucose

$\mathrm{HCl}$

$\mathrm{H}_{2} \mathrm{O}_{2}$

Histoacryl Topical Skin Adhesive

Leibovitz L-15 medium

Maleic acid

Methanol

$\mathrm{MgCl}_{2}$

$\mathrm{NaCl}$

NBT/BCIP tablets

Tissue-Tek O.C.T. compound

Orange G

Paraformaldehyde (PFA)

PBS pH 7.2

Proteinase $\mathrm{K}$

Salmon sperm DNA

Sodium dodecyl sulfate (SDS)

Sodium chloride

Sodium citrate

Sucrose

Tris
Carl Roth $\mathrm{GmbH}$

Applichem GmbH

Fermentas $\mathrm{GmbH}$

Carl Roth $\mathrm{GmbH}$

Carl Roth $\mathrm{GmbH}$

Carl Roth $\mathrm{GmbH}$

Carl Roth $\mathrm{GmbH}$

B. Braun $\mathrm{GmbH}$

Applichem GmbH

Carl Roth $\mathrm{GmbH}$

Carl Roth $\mathrm{GmbH}$

Carl Roth $\mathrm{GmbH}$

Carl Roth $\mathrm{GmbH}$

Roche Diagnostics GmbH

Sakura Finetek $\mathrm{GmbH}$

Applichem $\mathrm{GmbH}$

Carl Roth $\mathrm{GmbH}$

Invitrogen $\mathrm{GmbH}$

Applichem GmbH

Applichem GmbH

Carl Roth $\mathrm{GmbH}$

Carl Roth $\mathrm{GmbH}$

Carl Roth $\mathrm{GmbH}$

Carl Roth $\mathrm{GmbH}$

Carl Roth $\mathrm{GmbH}$ 
Triton X-100

Trizol reagent

Tryptone

Tween-20

Yeast extract
Carl Roth $\mathrm{GmbH}$

Invitrogen $\mathrm{GmbH}$

Carl Roth $\mathrm{GmbH}$

Carl Roth $\mathrm{GmbH}$

Applichem GmbH

\subsubsection{Plasmids}

\begin{tabular}{ll}
\hline Vector name & Supplier \\
\hline pCRII-TOPO & Invitrogen GmbH \\
pCAGEN & Addgene \\
Hb9::Cre & Lee et al.,2004 \\
pCAGGS::T2TP & Sato et al., 2007 \\
pT2K-CAGGS-EGFP & Sato et al., 2007 \\
\hline
\end{tabular}

\subsection{Animals}

According to the German "Tierschutzgesetz" (BGBI. I S. 1206, 1313), in ovo chick experiments do not require animal protocol permission. The experiments were performed on mice using procedures approved by the animal care committee of the Bezirksregierung Braunschweig (permit no. G42/08 and no. T9.07), Germany. Animals were fed ad libitum and were maintained on a 12-hour light-dark cycle by the animal facility of the ENI-Göttingen under the control of a veterinarian.

\subsubsection{Mouse lines}

CD-1 mice [Crl: CD-1 (ICR) BR] were obtained from the animal facility of the ENI-Göttingen. Olig2 ${ }^{C r e}$ transgenic mice were a gift from Dr. Thomas M. 
Jessell, Columbia University (Dessaud et al., 2007). Err2 floxed mice were purchased from Jackson Laboratories and were originally produced by Dr. Jeremy Nathans (Chen and Nathans, 2007). Mice homozygous for Err2 floxed allele possess loxP sites flanking exon 2 of the Err2 gene and are viable and fertile. These mutant mice were bred to $O \operatorname{lig} 2^{\text {Cre }}$ mice that express Cre recombinase in motor neuron progenitors at E8.5, resulting in offsprings which have exon 2 (containing the initiator methionine codon and encoding the $\mathrm{N}$-terminal 132 amino acids) deleted in the motor neurons.

\subsubsection{Mouse PCR genotyping}

Mouse genomic DNA was extracted from $5 \mathrm{~mm}$ long mice tail by incubation of the biopsy in $500 \mu \mathrm{l}$ tissue digest buffer overnight at $56^{\circ} \mathrm{C}$. The mixture was purified by centrifugation for 10 minutes at $16000 \mathrm{~g}$. Subsequently, supernatant from each sample was transferred into a new tube and mixed with $500 \mu \mathrm{l}$ isopropanol and centrifuged for another 10 minutes at $16000 \mathrm{~g}$. Afterwards, all supernatants were discarded and the pellet was washed with $500 \mu \mathrm{l}$ of $70 \%$ ethanol and centrifuged again for 10 minutes at $16000 \mathrm{~g}$. The DNA was air-dried and resuspended in $50 \mu \mathrm{l} \mathrm{H}_{2} \mathrm{O}$. PCR genotyping was performed with following primer sequences: Err2 forward primer 5'-GGGGGCCTTGGTCTACAGGTCTAGT-3', Err2 reverse primer 5'-TCTTTCTACGGCGTTTCAGGGACC-3', Cre forward primer 5'-GCTAAGTGCCTTCTCTACACCTGC-3', and Cre reverse primer 5'-GGAAAATGCTTCTGTCCGTTTG-3'. PCR reactions were performed in 25 $\mu \mathrm{l}$ volume. The reaction solution consisted of $1 \mathrm{U}$ of DreamTaq DNA polymerase, $2.5 \mathrm{mM}$ of each dNTP, $1 \mathrm{mM}$ of $\mathrm{MgCl}_{2}, 10 \mathrm{pmol}$ of both the forward, reverse primers, and $50 \mathrm{ng}$ of genomic DNA. The reaction thermal 
program composed of initial denaturation at $95^{\circ} \mathrm{C}$ for 90 seconds, and then 35 extension cycles including $95^{\circ} \mathrm{C}$ for 30 seconds, $60^{\circ} \mathrm{C}$ for 30 seconds, $72^{\circ} \mathrm{C}$ for 30 seconds, and the final synthesis at $72^{\circ} \mathrm{C}$ for 5 minutes.

\subsubsection{Retrograde labeling of motor neurons}

p0 mice were anaesthetized by hypothermia for 5 minutes. The regions around the muscles of interest were cleaned with $70 \%$ ethanol. The skin was incised with microscissors at appropriate areas to expose the target muscles: rectus femoris and soleus muscles (McHanwell and Biscoe, 1981; Pun et al., 2002). A glass microcapillary was filled with Alexa Fluor 488 or 555 conjugates CTB solution, and then was injected into target muscles. Subsequently, the closure of cutting wounds was achieved with Histoacryl topical skin adhesive. After injections, the mice were placed on a humidified incubation chamber at $35^{\circ} \mathrm{C}$. Retrograde tracing was performed for at least $7-8$ hours until they were sacrificed.

\subsubsection{Isolation of retrogradely labeled motor pools}

All plastic consumables and instruments should be DNase- and RNasefree. Spinal cords were obtained from mice sacrificed by decapitation were directly transferred onto silicone dish filled with ice cold DEPC-PBS. The spinal cord was fixed on the dish using insect pins. Under Olympus SZX16 fluorescence stereomicroscope, the retrogradely traced motor pools were dissected with microscissors and tungsten microblade. 


\subsubsection{Microarray}

Microarray sample hybridization and quantification were done by Dr. Lars Wittler and Dr. Philip Grote at the Max Planck Institute of Molecular Genetics Berlin. Briefly, total RNA of motor pools was extracted by Trizol reagent according to standard manufacturer's protocol, measured for concentration and quality control, before storing in $-80^{\circ} \mathrm{C}$ until use. RNA quality and concentration was determined with a ND-1000 spectrophotometer (NanoDrop). MouseRef-8 v2.0 expression beadchip microarrays, including approximately 25600 well-annotated RefSeq transcripts, over 19100 unique genes probes were used. RNA samples were prepared for microarray analysis using the Illumina TotalPrep RNA Amplification Kit. To synthesize the first cDNA strand, total RNA were reverse transcribed using T7 Oligo (dT) Primers, dNTP Mix, RNase inhibitor, first strand buffer and ArrayScript enzyme. All samples were incubated at $42^{\circ} \mathrm{C}$ for 2 hours. The second cDNA strand synthesis reaction was performed by DNA polymerase and RNase $\mathrm{H}$. After incubation at $16^{\circ} \mathrm{C}$ for 2 hours, double-stranded cDNA was purified and biotinylated cRNA was synthesized by in vitro transcription with T7 RNA polymerase and biotin-NTP, purified and its concentration and quality were assessed by NanoDrop.

\subsection{Mouse behavioral testing}

\subsubsection{Running endurance trail}

Forced treadmill running of 50 days old control and Olig2 $2^{\text {Cre }} ;$ Err2 $^{\text {loxP/loxP }}$ mice transgenic mice was performed as follows. All animals were pre-trained on the treadmill for 5 minutes at $7 \mathrm{~m} / \mathrm{min}$ for 2 days. On the next day, mice were forced to run at $8 \mathrm{~m} / \mathrm{min}$ for 30 minutes, then $9 \mathrm{~m} / \mathrm{min}$ for 15 minutes, followed by $10 \mathrm{~m} / \mathrm{min}$ for 15 minutes. Finally, the speed was gradually 
increased by $1 \mathrm{~m} / \mathrm{min}$ every 10 minutes until the mouse reached exhaustion. The stop point was reached when a mouse received repeatedly electrical shocks for more than 5 seconds.

\subsubsection{Grip strength assay}

Each 50-day-old animal was suspended by its tail and grasped a bar connected to the grip strength meter (Columbus Instruments). After the animal front paws grasped the bar it was moved slowly apart at a consistent speed. Hindlimbs are kept free from contact with the grid; the animal is brought to an almost horizontal position and pulled back gently but steadily until the grip is released. The maximal force of animals was recorded. Each animal should undergo 5 trials testing and only the highest value was taken for further analysis.

\subsection{Molecular biology}

\subsubsection{Molecular cloning}

The methods described in this section were taken from Molecular cloning : a laboratory manual (Sambrook and Russell, 2001) unless otherwise stated. Competent E. coli cells of the Top10 strain (Invitrogen $\mathrm{GmBH}$ ) were used in all cloning experiments. They were store at $-80^{\circ} \mathrm{C}$ until use. Digested vector and insert DNA concentration were determined and reaction mixtures were prepared with molar vector to insert concentrations of 1:3. An equal amount of DNA ligation mix was added to the vector-insert reaction. The reaction was incubated at $16^{\circ} \mathrm{C}$ for a minimum of 2 hours before transformation. E. coli was transformed by adding $100 \mu \mathrm{l}$ of competent cells to a maximum amount of 10 $\mu \mathrm{l}$ ligated DNA mix. The cells were incubated on ice for 30 minutes, 
heat-shocked at $42^{\circ} \mathrm{C}$ for 1 minute and placed on ice again for 1 minute before adding $1 \mathrm{ml}$ of antibiotic-free LB medium. They were then incubated in the Eppendorf thermomixer at $37^{\circ} \mathrm{C}$ for 1 hour. The cells were subsequently centrifuged at $3000 \mathrm{~g}$ for 2 minutes, and the supernatant was discarded before being plated on an agar plate containing Ampicillin. The agar plate was then incubated at $37^{\circ} \mathrm{C}$ for $16-18$ hours. E. coli cells were grown from single colonies on agar plates or bacterial stocks. A toothpick was used to pick a single colony of bacteria and the toothpick was cultured in $10 \mathrm{ml}$ of LB medium containing Ampicillin at $37^{\circ} \mathrm{C}$ overnight with $180 \mathrm{rpm}$ shaking. $0.5 \mathrm{ml}$ of $E$. coli overnight culture was mixed with $0.5 \mathrm{ml} \mathrm{100 \%} \mathrm{glycerol} \mathrm{in} \mathrm{a} 1.5 \mathrm{ml}$ tube and vortexed. The bacterial stock was stored at $-80^{\circ} \mathrm{C}$ until future use. Small-scale plasmid isolation was performed with $5 \mathrm{ml}$ of $E$. coli overnight culture using Fermentas Miniprep Kit according to kit's manual. Extracted DNA was eluted in $50 \mu$ of elution buffer. Large-scale plasmid isolation (for chick electroporation purposes) was performed with $200 \mathrm{ml}$ of $E$. coli overnight culture using Qiagen Plasmid Maxi Kit according to manufacturer's instructions. The concentration of the extracted DNA was adjusted to $1.5 \mu \mathrm{g} / \mu \mathrm{l}$ or $3.0 \mu \mathrm{g} / \mu \mathrm{l}$ using an appropriate volume of elution buffer.

\subsubsection{In situ hybridization}

In situ hybridization antisense probes were generated as follows: cDNA templates for antisense probes were synthesized using RT-PCR (Bio-Rad iScript cDNA Synthesis Kit). Total RNA from chick HH stage18 spinal cord and limbs was prepared using the Trizol reagent for RNA Isolation. Primers sequences for target cDNA sequence amplification were designed using the online program, Primer3 (http://frodo.wi.mit.edu/), and retaining using the 
default parameters, with the exception of defining probe size set to amplify 600-800 base pair gene fragments. Primers sequences for all in situ probes synthesized are listed in Table 1. The in situ probe fragments were amplified by PCR using the following settings: denaturation at $95^{\circ} \mathrm{C}$ for 60 seconds, and then 30 cycles including $95^{\circ} \mathrm{C}$ for 30 seconds, $58^{\circ} \mathrm{C}$ for 30 seconds, $72^{\circ} \mathrm{C}$ for 60 seconds, and the final synthesis at $72^{\circ} \mathrm{C}$ for 5 minutes. The PCR products were analyzed by $0.8 \%$ agarose gel electrophoresis. Correct PCR fragments were excised and purified by using QIAquick Gel Extraction Kit following QIAGEN standard protocol. Purified PCR products were subcloned into pCRII-TOPO vector using the TA-Cloning Kit according to the manufacture's protocol. Positive clones were selected on solid medium containing Ampicillin and verified by automated sequencing (QIAGEN Sequencing Service).

Table 1. Primers sequences for antisense riboprobe

\begin{tabular}{cccc}
\hline $\begin{array}{c}\text { Gene } \\
\text { Symbol }\end{array}$ & $\begin{array}{c}\text { NCBl Accession } \\
\text { number }\end{array}$ & Forward Primer & Reverse Primer \\
\hline Chat & NM_204610.1 & AACTTCCAGTCCCACCACTG & TTCCATCAGTCGTCAGCAAG \\
Err2 & XM_001235146.1 & AGTGCCATCATGGAGGATTC & CAGTTGTAGGATGGCCAGGT \\
Err3 & NM_001007081.1 & CATGAATGGGCATCAGAATG & CTCTGTCTGCCAGGTCACAA \\
\hline
\end{tabular}

The mouse Err2 and Calca probes have been described previously (Mitsunaga et al., 2004; Enjin et al., 2010).Probes were synthesized using T7, T3 or Sp6 RNA polymerases by DIG RNA labeling Kit according to the manufacturer's specifications. Probes were controlled and quantified using the Nanodrop ND-1000 Spectrophotometer. Chick and mouse spinal cords were 
isolated and fixed in $4 \%$ PFA dissolved in PBS for 24 hours on a shake at $4{ }^{\circ} \mathrm{C}$, equilibrated with $30 \%$ sucrose in PBS, embedded in O.C.T., and stored at $-80^{\circ} \mathrm{C}$. Afterwards, $30 \mu \mathrm{m}$ spinal cord sections were collected on Superfrost glass slides, using a Leica cryostat (CM1510S, Leica Microsystems). Slides were dehydrated in a graded series of methanol washes before storage in $100 \%$ methanol at $-20^{\circ} \mathrm{C}$. Sections were re-hydrated by successive washes in methanol and PBS. To remove endogenous alkaline phospphase activity, tissue sections were then bleached in $6 \% \mathrm{H}_{2} \mathrm{O}_{2}$. After three times PBST wash for 5 minutes each, sections were digested with $10 \mu \mathrm{g} / \mathrm{ml}$ of Proteinase $\mathrm{K}$ diluted in PBS for 10 minutes. Sections were post-fixed in 4\% PFA in PBS for 5 minutes and washed three times in PBS for 5 minutes each. Sections were dehydrated in a graded series of ethanol solution. Slides were air-dried the slides on Wattman Paper. Sections were incubated in hybridization buffer containing DIG-labeled riboprobes. Hybridization was performed overnight (16-18 hours) in $65^{\circ} \mathrm{C}$. After hybridization, slides were immersed in wash buffer I-III for 30 minutes each, before incubation in $2 \%$ blocking reagent for 1 hour at RT. Sections were incubated with 1:2500 diluted anti-digoxigenin alkaline phosphatase conjugated antibody in MBST at $4^{\circ} \mathrm{C}$ overnight. Unbound antibody was washed away by several washes of MBST. Slides were incubated with NTMT at RT for 10 minutes. In situ signals were developed by BM purple AP substrate solution (Roche Diagnostics) at RT in dark. The reaction was stopped by washing the slides with $\mathrm{H}_{2} \mathrm{O}$. After the color has fully developed, slides were mounted with cover slips using 50\% glycerol in PBS. 


\subsection{Chick In ovo electroporation}

Fertilized chick eggs (Lohmann LSL strain) were purchased from Geflügelzucht-Horstmann $\mathrm{GmbH}$. These eggs were incubated in the humidity chamber (Hemel-Brutgeräte $\mathrm{GmbH}$ ) until embryos reached HamburgerHamilton $(\mathrm{HH})$ stage 18. The Hb9::Cre vector was a gift from S. Pfaff. The expression vector consisted of a pCAGGS promoter derived from the pCAGEN vector, followed by three SV40 pA signals flanked between two loxP sites, V5-tag transgene expression cassette, IRES (Internal ribosome entry site), fluorescent reporters mGFP, SynGFP, and NLSeGFP), a terminating SV40 pA signal, and were inserted in this expression vector. A small hole was made in the shell on the side of the air chamber, and then $5 \mathrm{ml}$ albumin was extracted from the egg using a syringe. An oval window was made in the eggshell. A micropipette was filled with $0.2 \%$ fast green/DNA solutions. The DNA mixture was injected into the neural tube and the embryo was kept moist by adding L-15 medium on top of the vitelline membrane. The DNA solution was electroporated into half of the chick spinal cord using ECM 830 electroporator (Havard Apparatus) conducted $25 \mathrm{~V}$ pulses (50 ms duration and $200 \mathrm{~ms}$ interval) by L-Shaped Genetrodes $(5 \mathrm{~mm}$ in length and $0.5 \mathrm{~mm}$ diameter). Subsequently, the eggs were sealed and put back to the incubator until desired stages of development were reached.

\subsection{Cryosectioning and immunostaining}

Mouse or embryonic chick spinal cord samples were fixed with 4\% PFA in PBS at $4^{\circ} \mathrm{C}$ for $6-8$ hours and dehydrated in $30 \%$ sucrose dissolved in PBS at $4^{\circ} \mathrm{C}$ on a shaker. The tissues were deemed to be ready for routine embedding in plastic embedding molds using O.C.T. on dry ice when they sunk to the 
bottom of the plate. O.C.T. blocks containing samples were stored at $-20^{\circ} \mathrm{C}$ until use. For cryosection, 30-60 $\mu \mathrm{m}^{2}$ sections were cut on a Leica cryostat (CM1510S, Leica Microsystems) at -18 to $-22^{\circ} \mathrm{C}$ and collected on Superfrost Plus microscope slides (Fisher). Slides were subsequently left to dry at RT and stored at $-20^{\circ} \mathrm{C}$ thereafter or used immediately. For immunostaining, slides were washed four times with PBS for 15 minutes each time to remove residual O.C.T. and to rehydrate samples. Slides were placed in a humidifed chamber prior to primary antibody addition. The primary antibodies were diluted in antibody staining solution and $500 \mu \mathrm{l}$ of solution was applied on each slide. Slides were then incubated at $4^{\circ} \mathrm{C}$ overnight (16-18 hours). Afterwards, slides were washed with PBS for 4 times, 5 minutes each at RT to rinse away unbound primary antibodies. Secondary antibodies were also diluted in antibody staining solution (1:1000) and $500 \mu$ of solution was used per slide. The slides were incubated at RT for 1 hour, and then washed again with PBS for 4 times, 5 minutes each to get rid of excess secondary antibodies and finally mounted with cover slips using $50 \%$ glycerol in PBS before visualization under fluorescence microscope.

\subsection{Tol2 system: long-term expression in chick embryo}

In order to achieve long-term transgene expression in late stage chick embryos, I employed the transgene integration into the chick genome by Tol2-transposon construct (Figure 25A). The pCAGGS::T2TP vector was a gift

from K. Kawakami. There are two specific recognition sites flanking the transgene sequences in Tol2 system. First, I used to the CMV enhancer to drive specific transgene specific expression in the chick spinal motor neurons. Sequences flanking V5 have been optimized to a Kozak consensus translation 
initiation site to increase the expression levels in eukaryotic cells. The V5 epitope tag enables us to visualize the candidate genes expression by immunostaining. The MCS is located in Tol2 construct between CMV enhancer and the 2AeGFP coding sequences. Candidate genes subcloned into the MCS will be translated as fusion proteins to N-terminus of 2AeGFP if these sequences are in the correct reading frame as 2AeGFP and there should not have stop codon in-between. The 2A peptide sequences have been recently used in multicistronic constructs as an alternative to the IRES. The $2 \mathrm{~A}$ protein cleavage was considered to be processed by the autoproteolytic reaction. However, a ribosomal-skip pathway was discovered and $2 \mathrm{~A}$ protein has been termed cis-acting hydrolase elements (CHYSEL). Unlike IRES, the advantage of $2 \mathrm{~A}$ peptide was demonstrated to quantitatively coexpress polyproteins and also works in various cell types. For mouse Err2 and Err3 expression constructs, total RNA of E18.5 spinal cord was extracted using Trizol reagent. Full length cDNAs were synthesis by PrimeScript $1^{\text {st }}$ cDNA synthesis Kit according to the manufacturer's protocol. For other expression vectors, VP16 activation domain and engrailed repressor domain were amplified from pActPL-VP16AD and CAG-EnR plasmids (Addgene), respectively. Subsequently, VP16 or EnR domains were either inserted in front of Err2 or Err3 by in-frame subcloning. The mouse coding sequences of Err2 and Err3 genes were cloned after PCR amplification using the following primers:

\begin{tabular}{ccc}
\hline $\begin{array}{c}\text { Gene } \\
\text { Symbol }\end{array}$ & Forward Primer & Reverse Primer \\
\hline Err2 & CATGCCATGGATGTCGTCCGAAGACAGGCACC & CATGCCATGGCA CCTTGGCCTCCAGCATCTCCAGG \\
Err3 & CATGCCATGGATGGATTCGGTAGAACTTTGC & CATGCCATGGGACCTTGGCCTCCAGCA TTTCC \\
\hline
\end{tabular}




\subsection{Motor neuron size distribution histograms}

The soma areas of motor neurons of chick or mouse lumbar spinal cords were quantified on z-stack of optical sections taken using Leica TCS/MP confocal laser scanning microscope (Leica Microsystems GmbH).These images were obtained at a magnification of 20X. Afterwards, images were processed and adjusted using ImageJ from NIH image and Adobe Photoshop CS3. Lumbar motor neurons were co-labeled with retrograde tracer CTB or eGFP upon overexpression of different vectors. Motor neurons were outlined in the confocal plane which displayed the maximum soma area cross-sectional area. The immunoreactivity values of NeuN, CTB, and eGFP in motor neurons were measured using Adobe Photoshop CS3. Soma size distribution histograms were represented for each graph by counting soma area cross-sectional areas in $50 \mu \mathrm{m}^{2}$ bins. Soma distribution graphs for each construct represent grouped data from at least 5 individual animals (exact numbers provided in the Results section). Cell size distributions and fluorescent intensity histograms were plotted using GraphPad Prism software.

\subsection{Image Acquisition}

Confocal fluorescence images were taken on a Leica TCS SP2 confocal laser scanning microscope coupled to a Leica DMIRE2 inverted microscope (Leica Microsystems GmbH) using 20X,40X oil-immersion, and 63X oil-immersion objective lenses. Fluorescent dye excitation at $488 \mathrm{~nm}, 561 \mathrm{~nm}$, and $633 \mathrm{~nm}$ was provided by Ar/Kr, DPSS (Diode-pumped Solid-State), and He-Ne lasers, respectively. The fluorescent dye detection channel (PMT, photomultiplier tube) bandwidth was adjusted to $505-550 \mathrm{~nm}, 572-640 \mathrm{~nm}$, and 655-720 nm for Alexa Fluor 488, Alexa Fluor 555, and Alexa Fluor 647, 
respectively. Six to ten consecutive single optical sections were obtained and were saved by Leica SP2 software. In double or triple labeling experiments, sequential scans in all the two/three channels were obtained with the PMT bandwidth settings described above. The saved images were imported to ImageJ software and were stacked in the z-dimension (maximum intensity projections). The stacked images were processed using Adobe Photoshop CS3 for adjustment of the levels, brightness/contrast, and match color functions. All the effects mentioned were applied uniformity across the whole images. Whole mount staining pictures were assembled in Adobe Photoshop CS3 using the automated photomerge option. In situ bright field signals were captured on Olympus IX81 microscope using 10X or 20X objective (transmitted light model).

\subsection{Statistics}

Prism 5.0 software was used for all statistical analysis unless otherwise stated. Two-tailed Student's $t$-test (unequal variance) was used for all data analysis, where applicable. Error bars in figures indicate standard deviation of mean (SEM). 


\section{Results}

\subsection{Expression profiling of functionally different motor neuron pools}

Within the spinal cord, transcription factors establish distinct neuronal subtypes by orchestrating precise spatial and temporal mechanisms (Shirasaki and Pfaff, 2002; Dasen and Jessell, 2009). However, molecular mechanisms underlying the functional specification of motor neuron subtypes (fast, slow, alpha-, beta-, and gamma-motor neurons) are still unknown. Each motor neuron innervates several muscle fibers in a single muscle and this functional connection is called a motor unit. A single muscle contains several hundred motor units. Each muscle fiber which is innervated by a single motor neuron is of the same type in a motor unit (Kandel et al., 2000). However, the muscle fiber composition of a single muscle is heterogeneous. There is no pure fast or pure slow muscle in the mouse musculature, except for muscles with a higher proportion of fast or slow muscle fibers. Therefore, two specialized muscles in mouse hindlimb were chosen in this study. In order to screen gene expression profiles from functional spinal motor neuron subtypes derived from E18.5 mice, the retrograde tracer cholera toxin subunit $B$ (CTB, the non-toxic isoform) was applied locally into the rectus femoris (predominantly "fast" muscle) and soleus muscle (predominantly "slow" muscle) (Figure 6) and 7-8 hours later, the retrogradely labeled fast or slow motor neuron pools were collected by microdissection. For each backfilled motor neuron pool, the mRNA was isolated and amplified from at least four independent experiments. Then, the biotinylated-cRNA from labeled motor neurons was hybridized on mouse Illumina gene arrays (MouseRef-8 v2 Expression BeadChip). The raw signal 
intensity values were further analyzed and processed using Illumina's Genome Studio Gene Expression Module. The Student's $t$-test was performed to check for the statistical significance and then a cutoff criterion ("fold change $>1.3$ fold" and "p-value < 0.05 ") was applied to select the candidate genes. Thereafter, I grouped the candidate genes into three categories: transcription factors, cell adhesion molecules, and ion channels. In this project, I mainly focused on the transcription factors since these factors are expected to play a prominent role in cell fate specification, including motor neurons. 

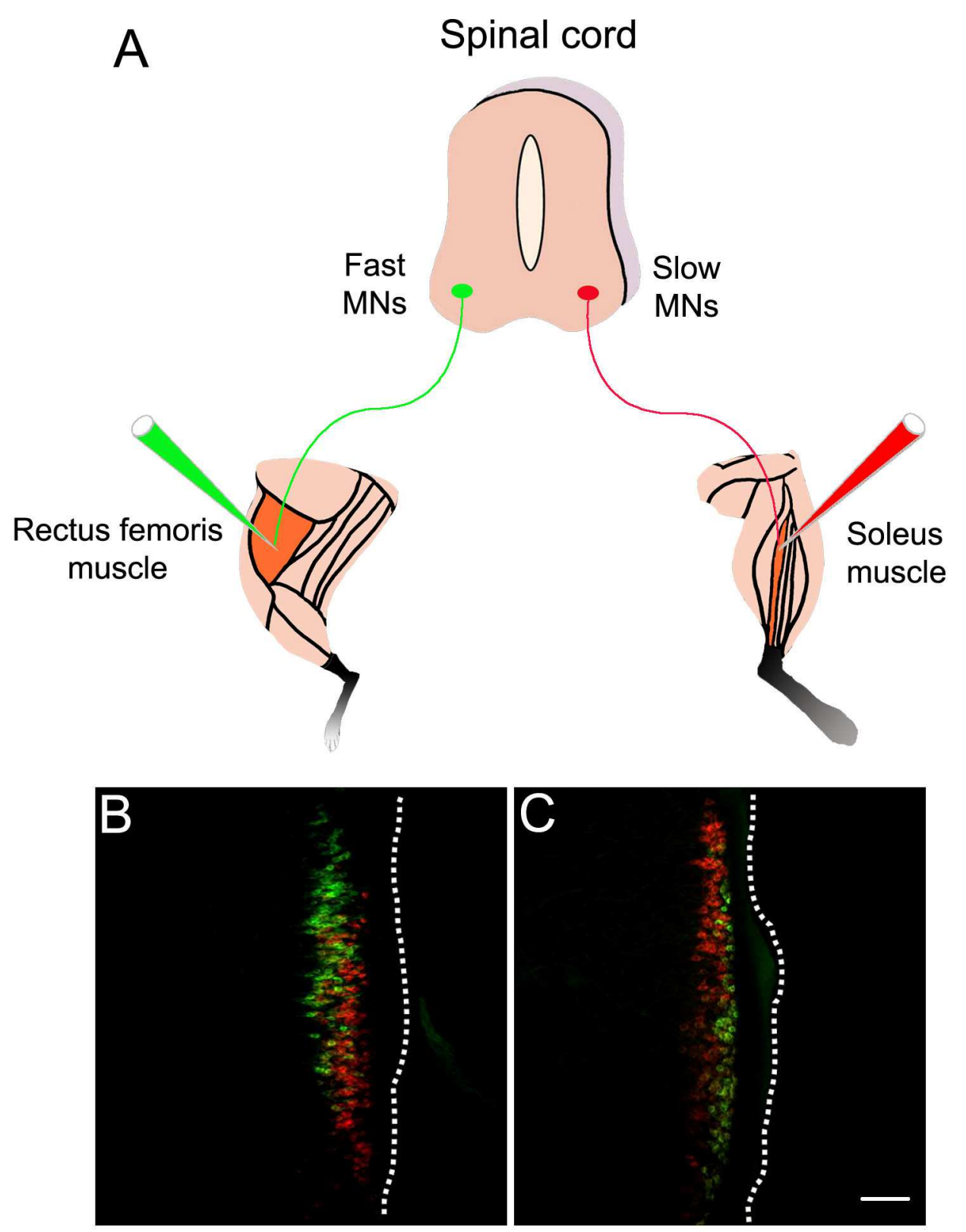

Figure 6. Schematic representation of the spinal motor neuron labeling procedure. (A) Alexa Fluor conjugated CTB is injected locally into rectus femoris and soleus muscles and then the fluorescence tracer is transported retrogradely to cell bodies of motor neurons. The labeled motor neurons were collected by fluorescent guided microdissection. (B) Whole spinal cord showing retrogradely traced motor neurons, 7-8 hours after local tracer application into rectus femoris and soleus muscles. Rectus femoris motor pool (green) and vastus medialis motor pool (red); (C) Whole spinal cord showing soleus motor pool (red) and extensor digitorum longus motor pool labeled with different Alexa Fluor conjugated CTB. Scale bar: 200 $\mu \mathrm{m}$ 
More importantly, it was expected that transcription factors would regulate ion channels and ion channel regulatory subunits that could determine specific firing properties of motor neurons. The heat map in Figure 7 shows the gene expression profile of a predominantly "fast" versus a predominantly "slow" motor pool obtained from microarray analysis in E18.5 mice (Figure 7).

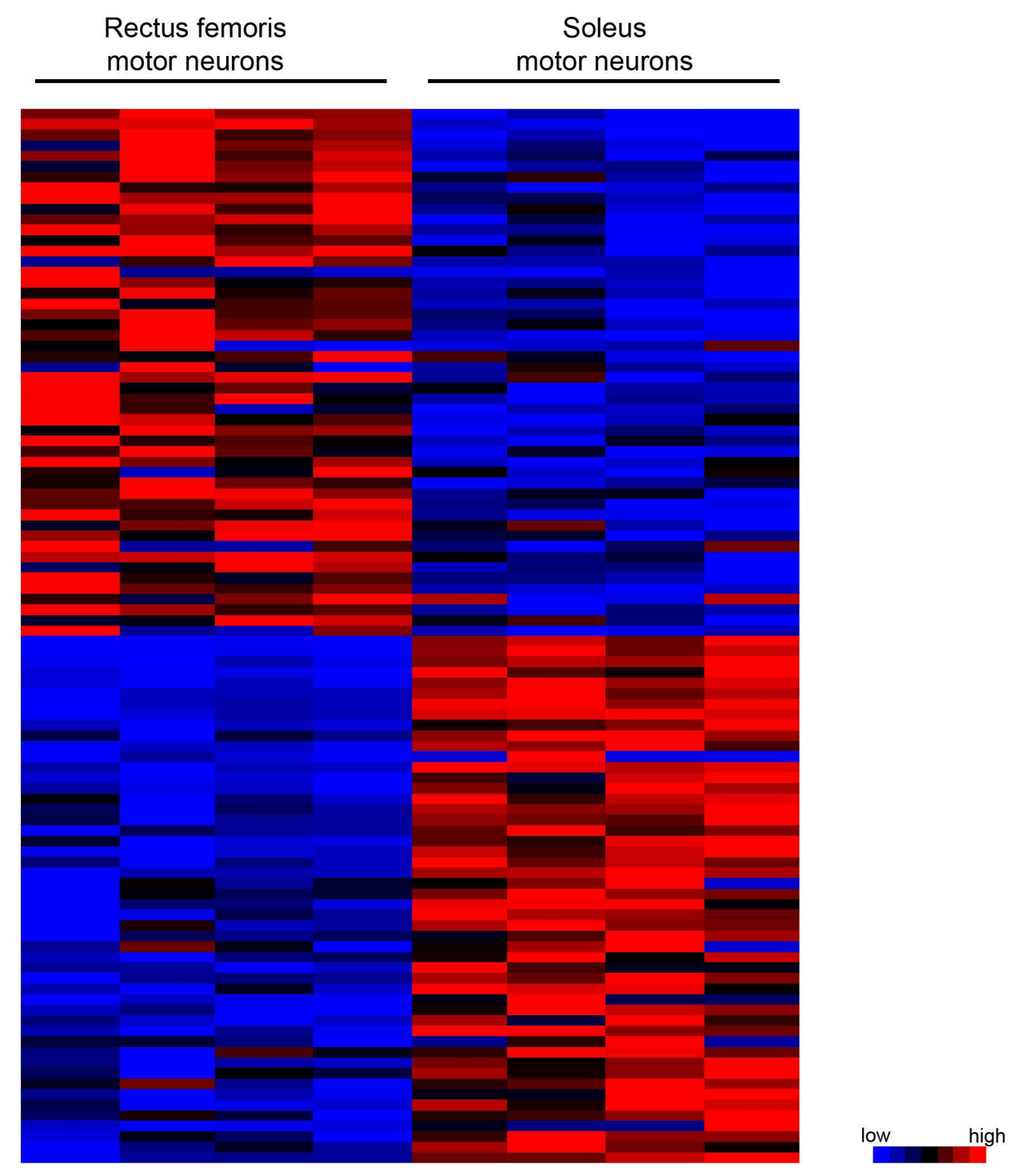

Figure 7. Gene expression profiles of rectus femoris and soleus motor neurons. The top 50 up- and down-regulation genes in rectus femoris and soleus motor neurons are listed. Each individual column in the heat map represents 4 animals. Red $=$ up-regulation; blue $=$ down-regulation, and black $=$ no differentially expression. 


\subsection{Candidate genes in functional motor neuron subtypes}

Further, the gene expression signatures of these motor pools were validated by immunostaining or in situ hybridization on embryonic and early postnatal lumbar spinal cord tissue. The transcription factor, Tshz3 is one of the candidate genes enriched in soleus motor neurons. Recently, it was revealed that Tshz3 plays an important role in neural circuitry by controlling the neural circuitry during breathing in mice. Mice lacking Tshz3 are postnatal lethal due to dysfunction of central respiratory rhythmogenesis (Caubit et al., 2010). In Drosophila melanogaster, the homologous teashirt gene regulates the development of the trunk (Roder et al., 1992). Based on the array data, there is a two-fold enrichment in the expression of Tshz3 by soleus motor neurons compared to rectus femoris motor neurons (Figure 8B). Furthermore, fluorescent immunostaining was combined with retrograde labelling from the rectus femoris and soleus muscles. The results show that Tshz3 transcription factor is expressed at higher levels in soleus motor neurons compared to rectus femoris motor neurons (Figure $8 \mathrm{~A}$ ). 


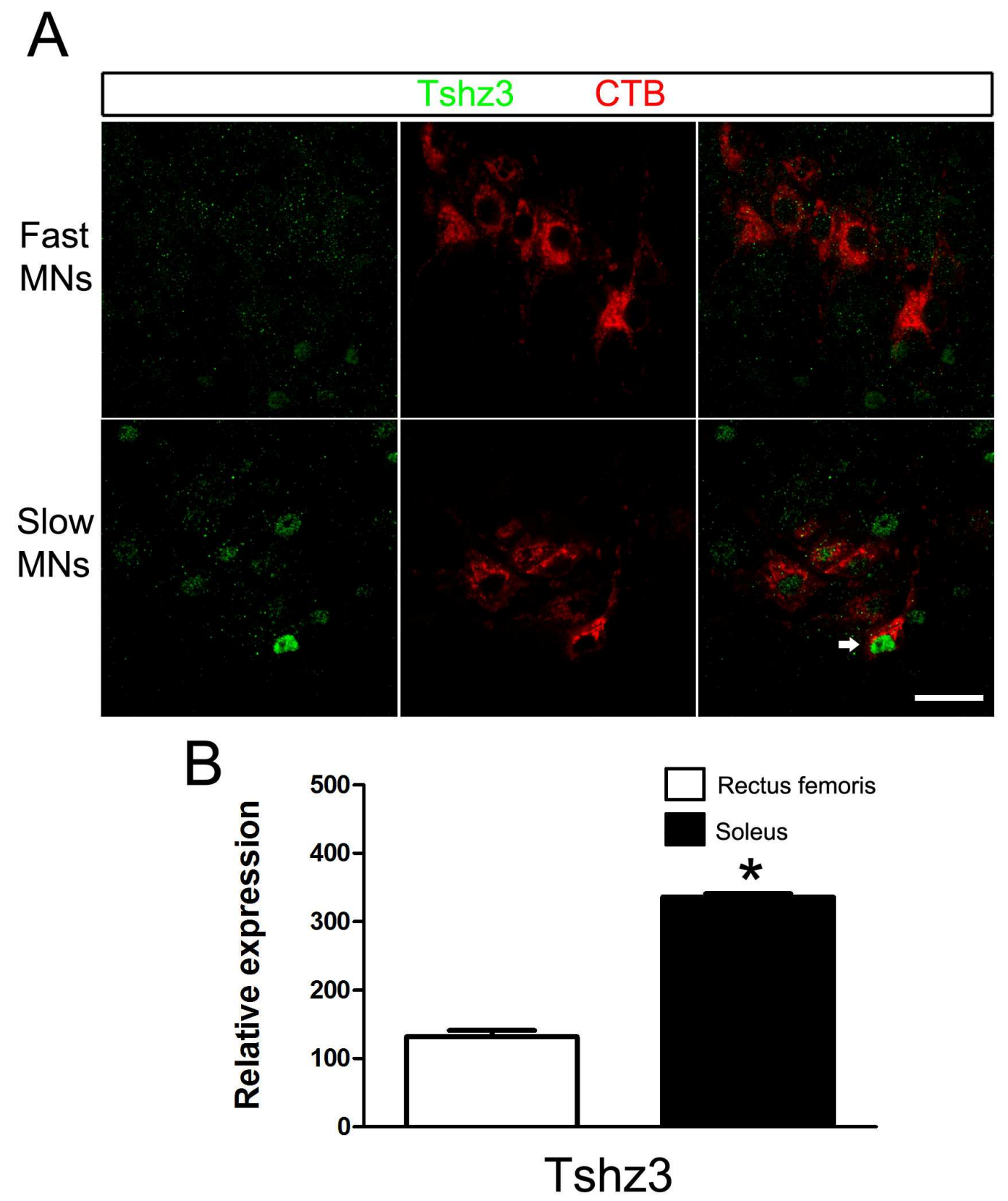

Figure 8. Tshz3 is strongly expressed by the soleus motor neurons. (A) Analysis of Tshz3 (green) in fast and slow motor neurons (red) at E18.5. Fast and slow motor neurons were traced retrogradely by intramuscular injection of rectus femoris and soleus muscles, respectively. (B) Microarray data indicates that the relative expression level of Tshz3 is higher in slow motor neurons compared to fast motor neurons $(n=4)$. Asterisk denotes significant difference (Student's $t$-test) ${ }^{*} p<0.01$. Scale bar: $50 \mu \mathrm{m}$. 
It has been reported that the neuropeptide, calcitonin gene-related peptide alpha (CGRP), encoded by the Calca gene plays a crucial role in a variety of physiological processes. Calca is widely expressed in the central and peripheral nervous systems and is expressed by a subpopulation of spinal motor neurons in chick and mice. Recently, it has been suggested that Calca and Chodl are expressed by fast motor neurons in the mouse (Enjin et al., 2010). From my microarray screening, it is clear that Calca and Chodl are highly enriched in the "fast" rectus femoris motor neurons, as compared to the "slow" soleus motor neurons (Figure 9C). My microarray results also demonstrate that Err2 is highly enriched (approximately four-fold) in rectus femoris motor neurons, as compared to soleus motor neurons. Previous studies have shown that Err2 has an important role in regulating placental development. Because of the abnormal development of chorion and trophoblasts, Err2 knockout mice die at mid-gestation (Luo et al., 1997). Conditional knockout mouse studies further showed that Err2 is essential for normal inner-ear development (Chen and Nathans, 2007). However, the function of Err2 transcription factor in spinal motor neurons needs to be elucidated. Results from in situ hybridization on early postnatal spinal cords (p5), showed a scattered expression pattern of Calca and Err2 in the ventral spinal cord (Figure 9A \& 9B and 9D \& 9E, respectively). 

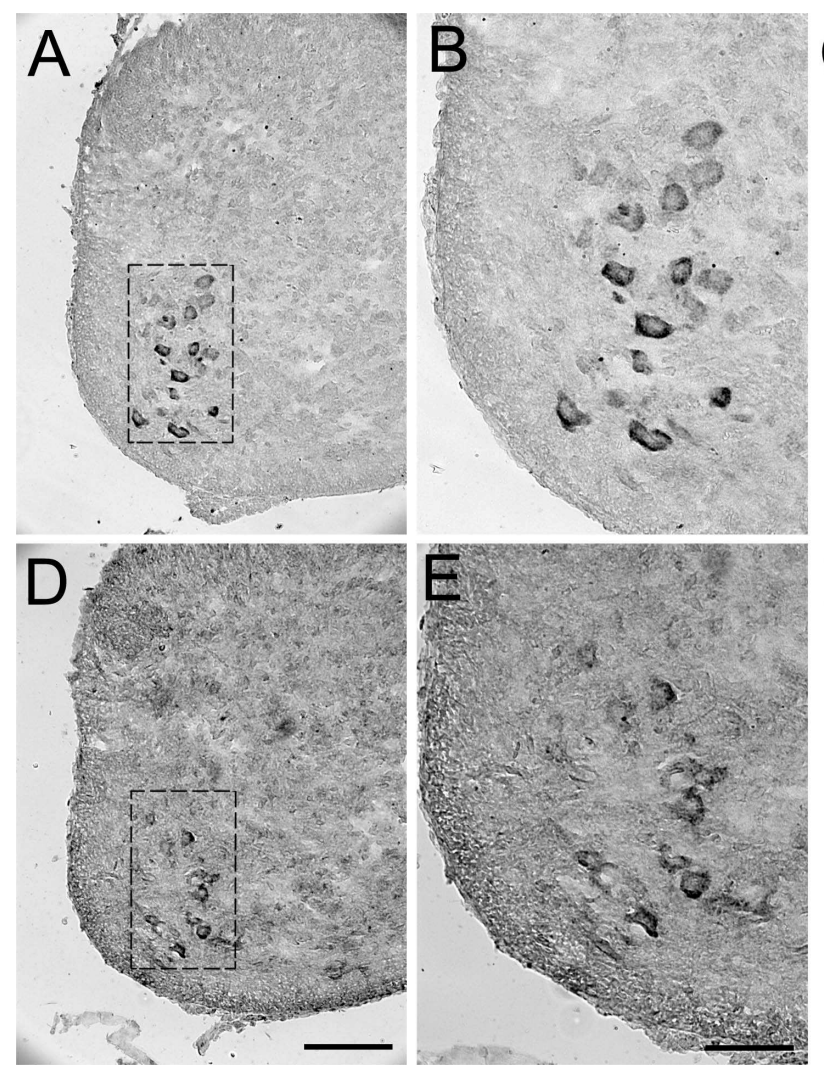

\section{C}

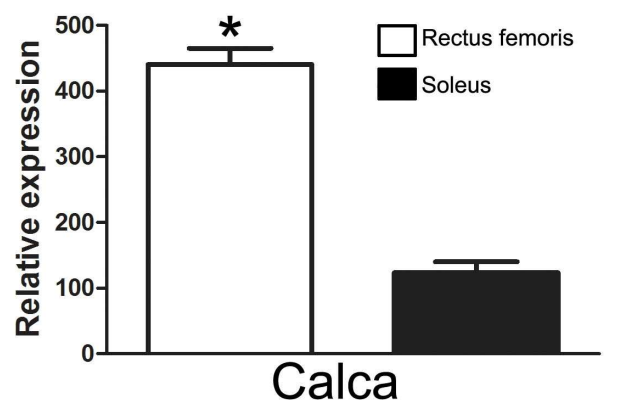

$\mathrm{F}$

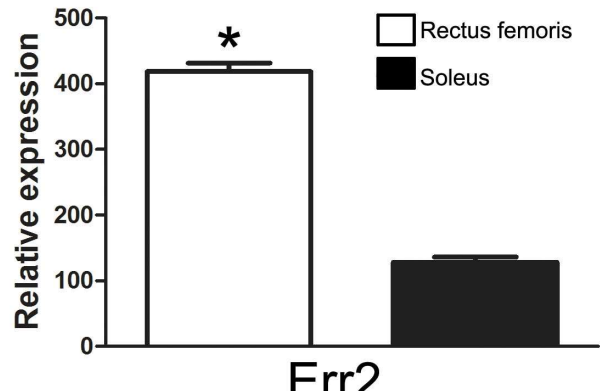

Figure 9. Calca and Err2 mRNA is highly enriched in rectus femoris motor neurons. In situ hybridization reveals a scattered expression of Calca ( $A$ and $B$ ) and of Err2 ( $D$ and $E$ ) in p5 spinal cord. $B$ and $E$ show high magnification of boxed area in $A$ and $D$, respectively. ( $C$ and $F$ ) Microarray data reveals that Calca and Err2 mRNA is highly enriched in fast motor neurons compared to that of slow motor neurons $(n=4)$. Asterisks indicate significant differences (Student's $t$-test) ${ }^{*} p<0.01$. Scale bar: (A and D) $100 \mu \mathrm{m}$; (B and E) $50 \mu \mathrm{m}$.

\subsection{Err2 expression is restricted to a small-sized motor neuron population in the lumbar spinal cord}

The ERR protein family has three members, namely Err1, Err2, and Err3, which bind to the estrogen response elements and regulate transcription (Tremblay and Giguere, 2007). However, these nuclear receptors do not bind to endogenous estrogens. These orphan receptors have been shown to play important roles in energy homeostasis and metabolism (Germain et al., 2006). For example, Err1 is expressed in various tissues that regulate fatty acid metabolism. Err1 mutant mice are viable but show less body weight and fat 
deposits in peripheral tissue. Importantly, Err1 null mice are resistant to high calorie food-induced obesity (Luo et al., 2003). In the cardiac tissue where Err3 is highly expressed, knocking out Err3 leads to electrophysiological defects and loss of control in cardiac conduction integrity (Alaynick et al., 2007). However, the functional role of estrogen-related receptor family in spinal motor neurons is still unclear. Err2 and Err3 have been shown to be expressed in the murine central nervous system, but not Err1 (Giguere, 1999). I used a commercial mouse monoclonal antibody to detect endogenous Err2 expression in the mouse spinal cord. Because the members of the ERR proteins have conserved protein sequences, I tested the specificity of the Err2 antibody. To examine this, mouse Err2 or Err3 expression constructs were transfected by in ovo electroporation into the chick neural tube at E3. In double immunofluorescent staining, Err2 antibody seems to specifically recognize Err2 protein, and does not cross-react with Err3 protein (Figure 10). 


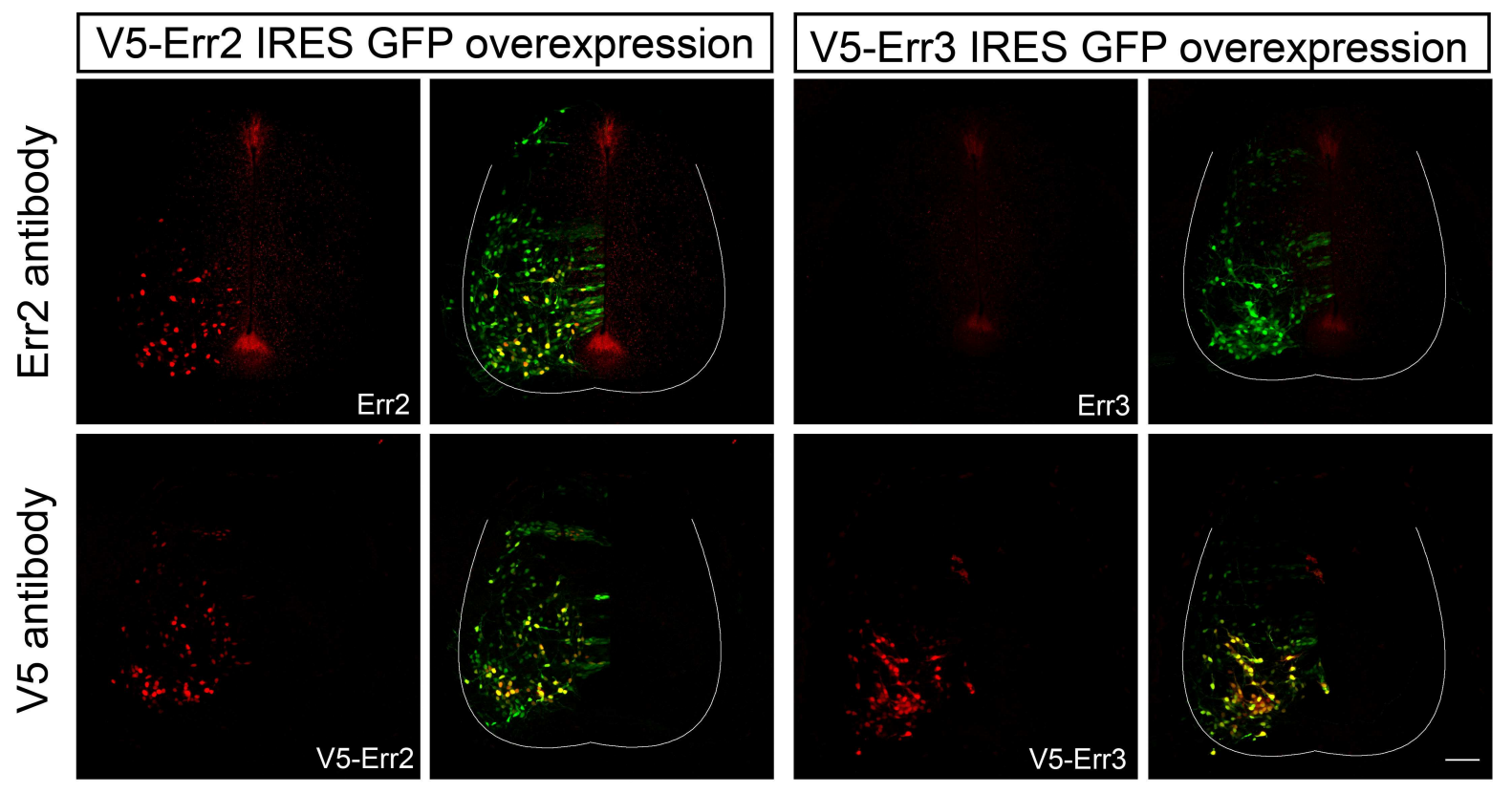

Figure 10. Assay to test the specificity of the Err2 antibody. Err2 or Err3 expression constructs were transfected by in ovo electroporation into the chick neural tube at E3. eGFP expression indicates the electroporated side of the spinal cord. Immunostaining using anti-Err2 and anti-V5 antibodies was performed on E6 chick lumbar spinal cord sections. The results show that the anti-Err2 antibody specifically recognizes Err2 protein and does not cross-react with Err3 protein. Scale bar: $100 \mu \mathrm{m}$.

Next, I analyzed the developmental changes of Err2 expression in embryonic and postnatal spinal cord. During mouse embryonic development, all postmitotic motor neurons initially express the homeodomain LIM protein Isl1. At E10.5, Err2 is also co-expressed by most Isl1-positive motor neurons (Figure 11). 


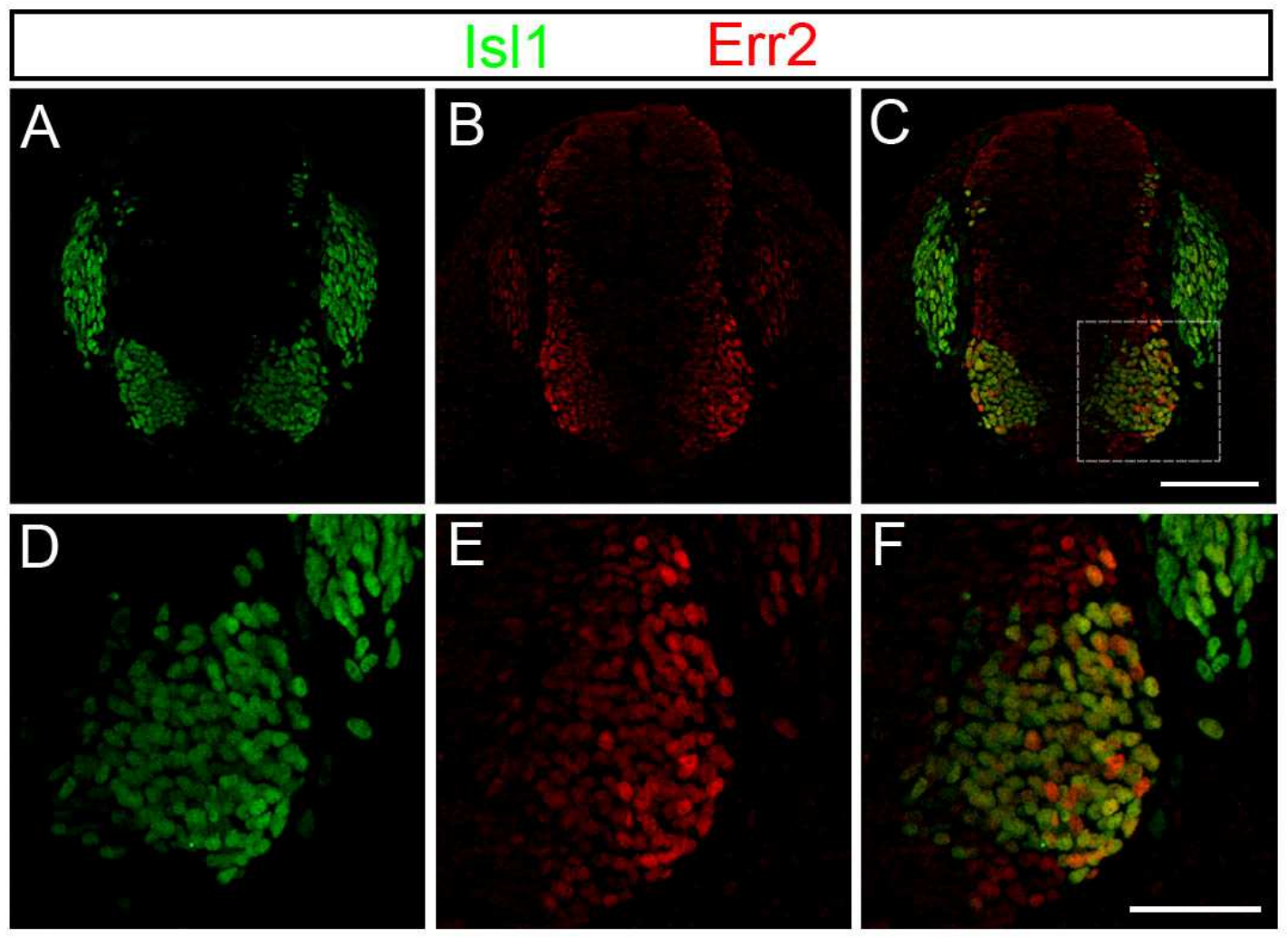

Figure 11. Err2 is broadly expressed in developing mouse motor neurons. Expression of Isl1 in motor and sensory neurons was detected with an anti-Isl1 antibody (green, A, C, D, and F). Expression of Err2 protein at E10.5 in embryonic mouse spinal cord was detected using an anti-Err2 antibody (B, C, E, and F). D, E, and F show high magnification of boxed area in C. Double immunostaining reveals co-localization of Isl1 and Err2 proteins in sensory and motor neurons (yellow in $C$ and F). Scale bar: (A, B, and C) $100 \mu \mathrm{m}$; (D, E, and F) $50 \mu \mathrm{m}$.

Spinal motor neurons are subdivided into large-sized alpha-motor neurons and small-sized gamma-motor neurons. Surprisingly, immunohistochemical analysis of p5 and p15 lumbar spinal cord demonstrated that Err2 is expressed most strongly in a subpopulation of relatively small-diameter motor neurons (Figure 12). 


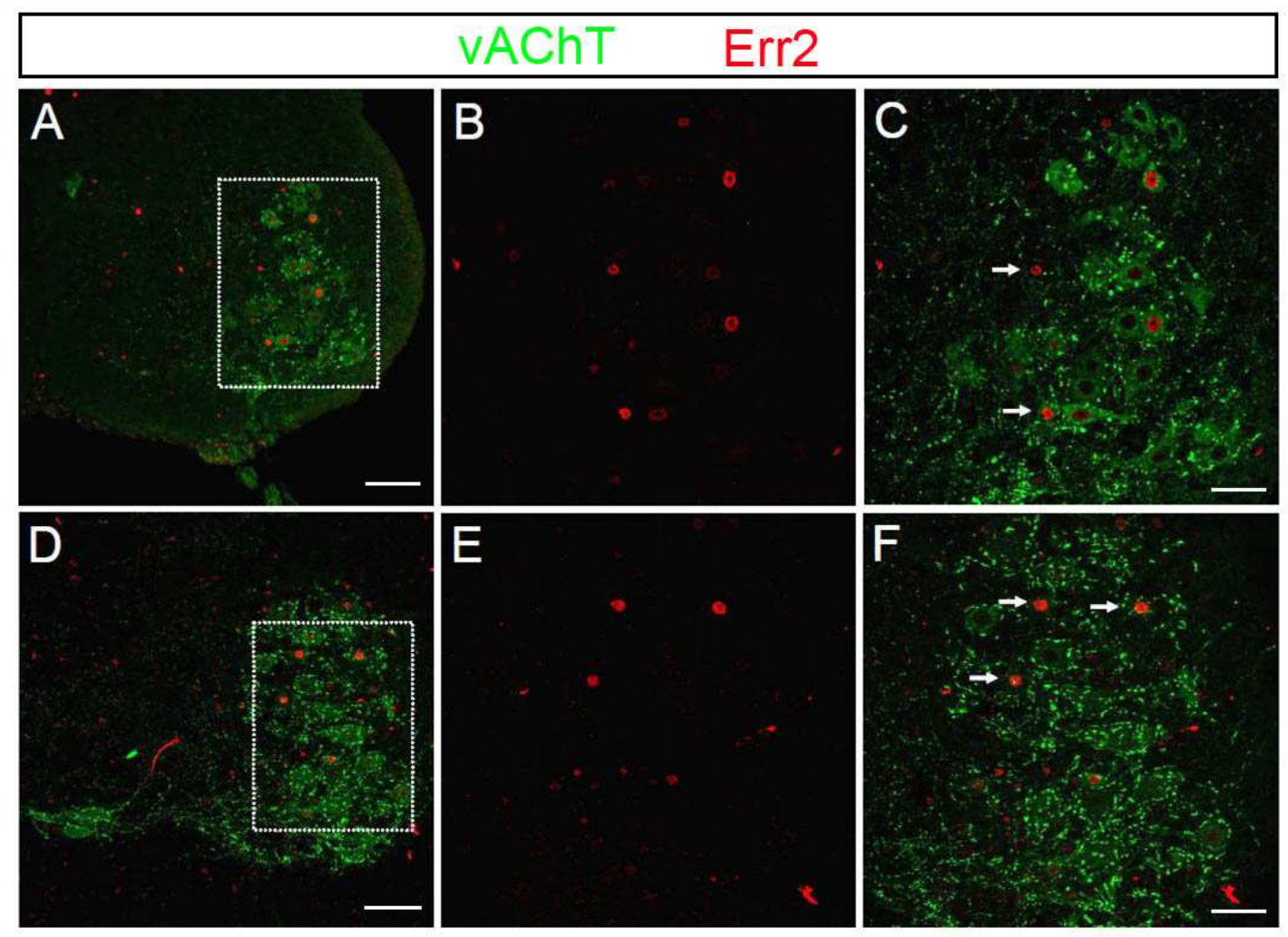

Figure 12. Small soma-sized motor neurons in the postnatal spinal cord are Err2 positive. (A) Confocal images of p5 spinal cord showing Err2 immunoreactivity (red) in vAChT-positive motor neurons (green). B and C show high magnification of boxed area in A. (D) In p15 spinal cord, Err2 expression is restricted to small-diameter spinal motor neurons. $E$ and $\mathrm{F}$ denote high magnification of boxed area in $\mathrm{D}$. Arrows indicate the small-sized motor neurons which are strongly Err2 positive (C and F). Scale bar: (A) $100 \mu \mathrm{m}$; (B and C) $50 \mu \mathrm{m}$; (D) $100 \mu \mathrm{m}$; (E and F) $50 \mu \mathrm{m}$.

To analyze Err2 expression in different spinal motor neuron pools, the fluorescent tracer CTB was injected into rectus femoris and soleus muscles in p1 mice. Retrograde labeling combined with immunostaining confirmed that small-sized motor neurons express high levels of Err2 (Figure 13A). At the same time, I also detected Err2 expression in thoracic and brachial region of spinal cord (data not shown). Err2 expressing cells were observed at all levels of the spinal cord, suggesting the Err2 expression is not specific to certain motor neuron pools. However, according to the microarray data, Err2 was found to be expressed at higher levels in rectus femoris motor neurons 
compared to soleus motor neurons. Quantitative analysis of Err2 protein expression intensity in motor neurons at p5 revealed that rectus femoris motor neurons indeed display a higher Err2 expression levels compared to soleus motor neurons (Figure 13B).

A
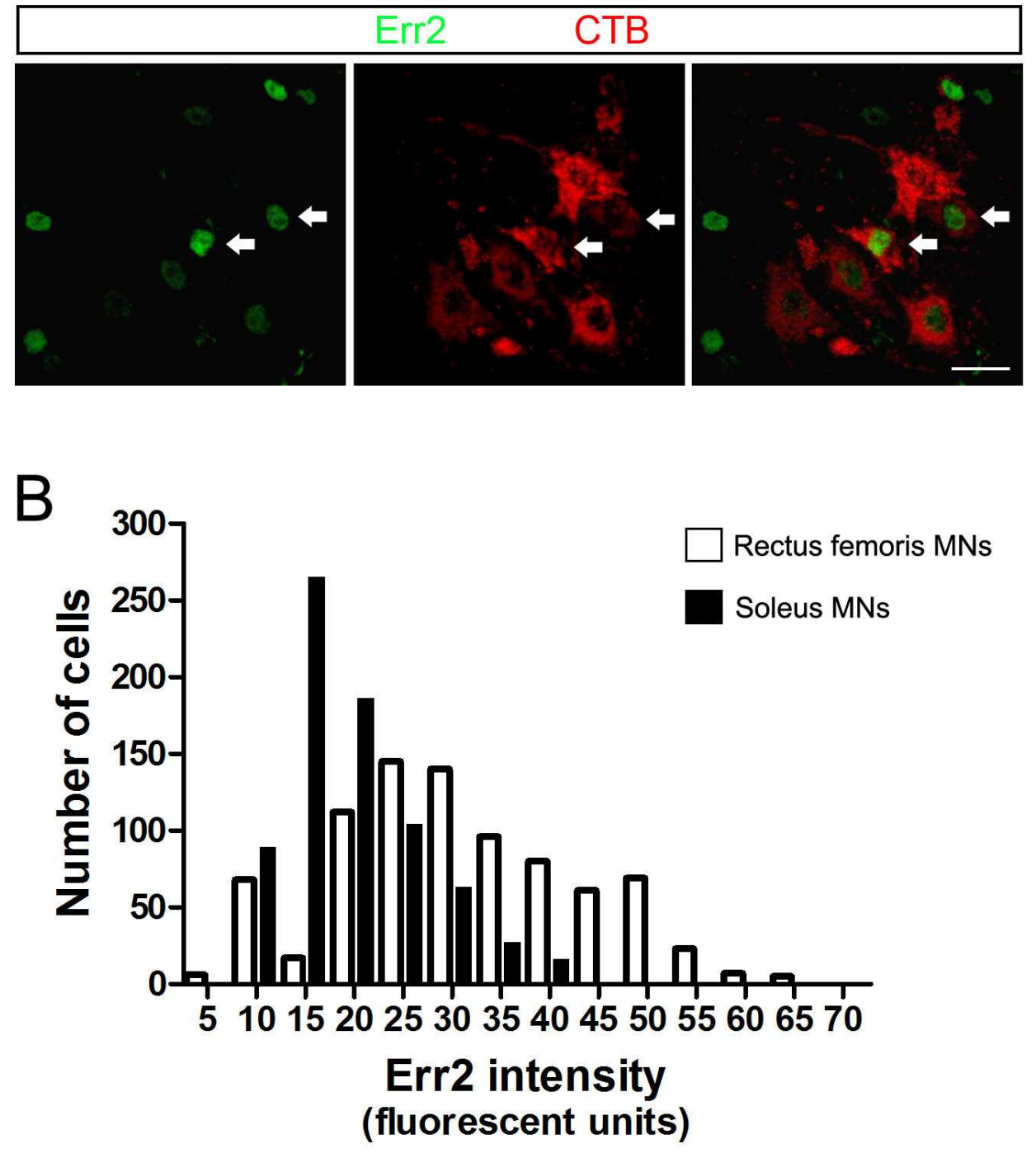

Figure13. Higher levels of Err2 protein in rectus femoris motor neurons when compared to soleus motor neurons. (A) In retrogradely labeled rectus femoris motor neurons (red), Err2 immunoreactivity (green) is restricted to small-sized motor neurons (arrows). (B) Analysis of Err2 expression intensity (arbitrary fluorescent intensity units) in rectus femoris and soleus motor neurons at p5. Err2 expression profile is higher in rectus femoris motor neurons (white column, $n=823$ cells) compared to soleus motor neurons (black column, $n=771$ cells). Scale bar: $50 \mu \mathrm{m}$. 
Spinal motor neurons can be broadly classified as alpha- and gamma-motor neurons. Alpha-motor neurons can be further classified as fastand slow-motor neurons according to the muscle fiber types that each subpopulation innervates (Manuel and Zytnicki, 2011). There are two major features distinguishing alpha- and gamma-motor neurons: (1) soma diameter and (2) vGlut1 synaptic contacts (Eccles et al., 1960; Friese et al., 2009). Basically, the soma size of gamma-motor neurons is significantly smaller than that of alpha-motor neurons. Moreover, only alpha-motor neurons but not gamma-motor neurons receive vGlut1-positive proprioceptive input from sensory neurons. It has recently been shown that transcription factor Err3 (closely related to Err2) is a molecular marker of spinal gamma-motor neurons (Friese et al., 2009). According to this study, gamma-motor neurons display high levels of Err3 expression and low levels of NeuN expression. I next correlated the expression of Err2 with NeuN and vAChT in the lumbar spinal cord. Interestingly, fluorescence triple-staining experiments showed that Err2 was detected in vAChT-positive motor neurons with relatively small soma area and lacking NeuN expression (Figure 14A-14C). Several studies have shown that p14 spinal motor neurons exhibit a bimodal soma size distribution. The small-diameter of Err2 ${ }^{\text {high }} / \mathrm{NeuN}^{\text {low }}$ neurons prompted us to quantity the soma size distribution of motor neurons in p15 lumbar spinal cord. By using molecular markers (NeuN $\left.\mathrm{N}^{\mathrm{high}} / \mathrm{vAChT}\right)$ for alpha-motor neurons and

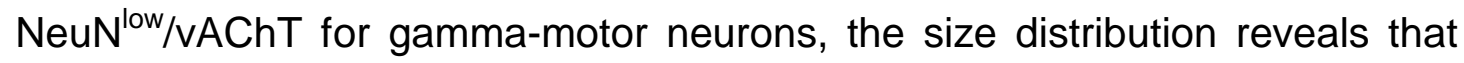
Err2 $2^{\text {high }} / \mathrm{NeuN}^{\text {low }}$ motor neurons display the same soma diameter characteristics as putative gamma-motor neurons (Figure 14D). 

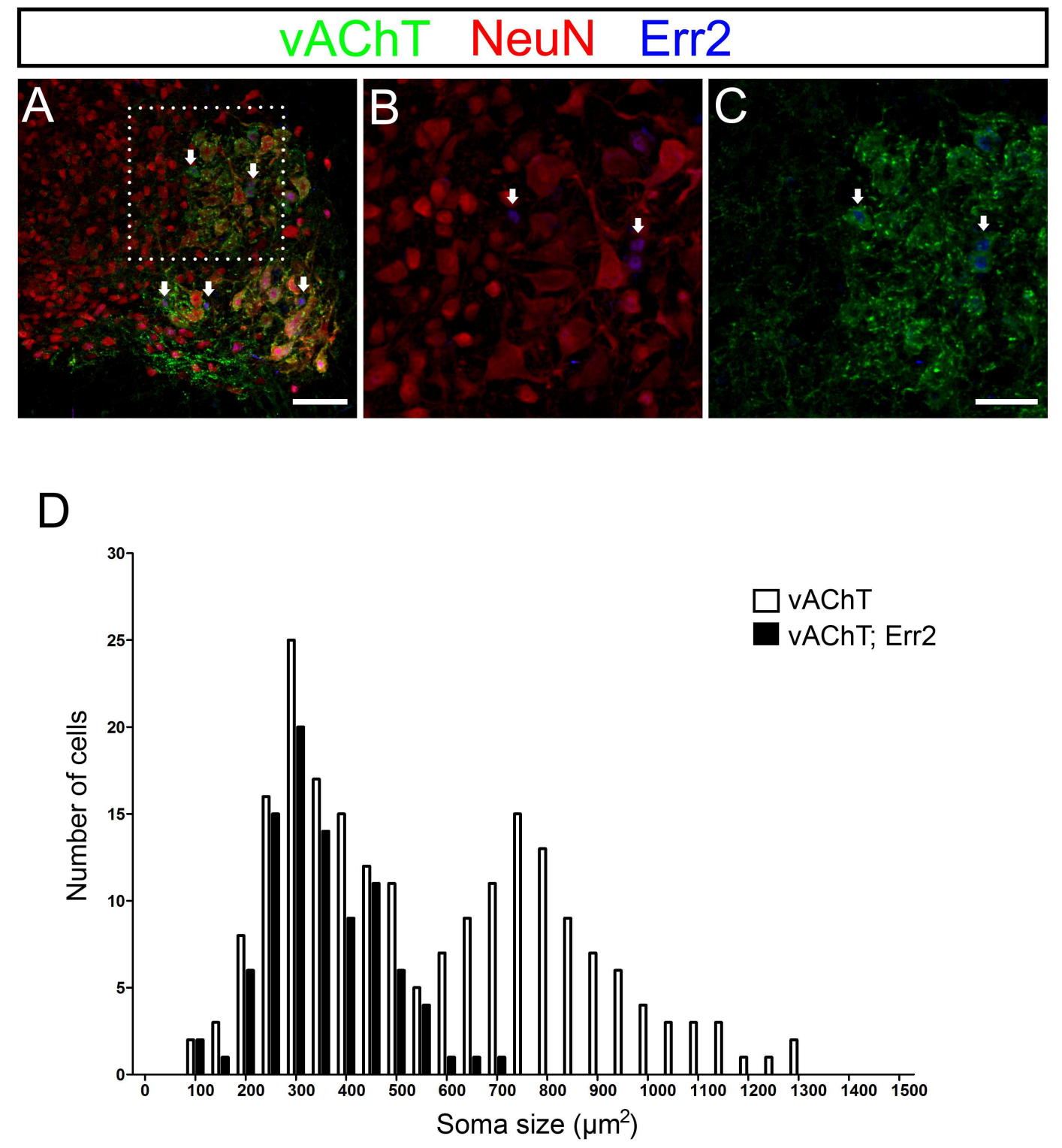

Figure 14. Gamma-motor neurons are Err2 ${ }^{\text {high }}$ and $\mathrm{NeuN}^{\text {low }}$ in postnatal spinal cord. (A) Alpha- and gamma-neurons are distinguished by expression of neuronal DNA binding protein NeuN. In the postnatal spinal cord, alpha-motor neurons have larger soma size and they express vAChT (green) and NeuN (red). However, gamma-motor neurons (arrows) have small soma size and they express vAChT and Err2 (blue). Boxed area in A is depicted at higher magnification in $B$ and $C$. (D) Soma size distribution profile of motor neurons in $\mathrm{p} 15$ spinal cord illustrate that Err2 ${ }^{\text {high }} / \mathrm{vAChT}$ motor neurons have soma size similar to that of gamma-motor neurons. Scale bar: (A) $100 \mu \mathrm{m}$; (B and C) $50 \mu \mathrm{m}$. 
Based on a number of criteria, it can be concluded that Err2 is a gamma-motor neuron marker. The differences in Err2 expression observed between rectus femoris and soleus motor pools at perinatal stages could reflect differences in the maturity and thus the timing of gamma-motor neuron specification, between these motor neuron pools (known to occur e.g. between extensor and flexor motor neurons). Alternatively, this could be due to an unexpected abundance of gamma-motor neurons in the rectus femoris motor pools.

To probe whether Err2 would function in the specification of gamma-motor neurons, I examined whether Err2 loss-of-function in spinal motor neurons affect the motor performance of mice in a series of behavioral tasks. Since the complete knockout of Err2 results in embryonic lethality (at E9.5, due to disruption of the chorion and placenta), to circumvent embryonic lethality, tissue-specific promoter-driven Cre lines were used to investigate the role of Err2 in spinal motor neurons. Err2 floxed mice crossed with a well-characterized Olig2-Cre line that drives expression of Cre recombinase protein in motor neuron progenitors results in deletion of Err2 specifically in motor neurons. Since Err2 was conditionally deleted in motor neurons, I checked whether this deletion affects motor behavior. Err2 is a gamma-motor neuron marker and perturbation of gamma-motor neurons formation might lead to impaired motor performance. However, grip strength analysis has revealed that the muscular strength of Err2 mutant mice was not affected (Figure 15A). When the mutant mice were subjected to forced-running until exhaustion on a treadmill, there was also no difference in running endurance in comparison to the controls (Figure 15B). Therefore, no difference in motor behavior was observed between control and Err2 mutant animals, which 
suggest a functional compensation of Err2 loss by genetic redundancy with the co-expressed Err3 in gamma-motor neurons.
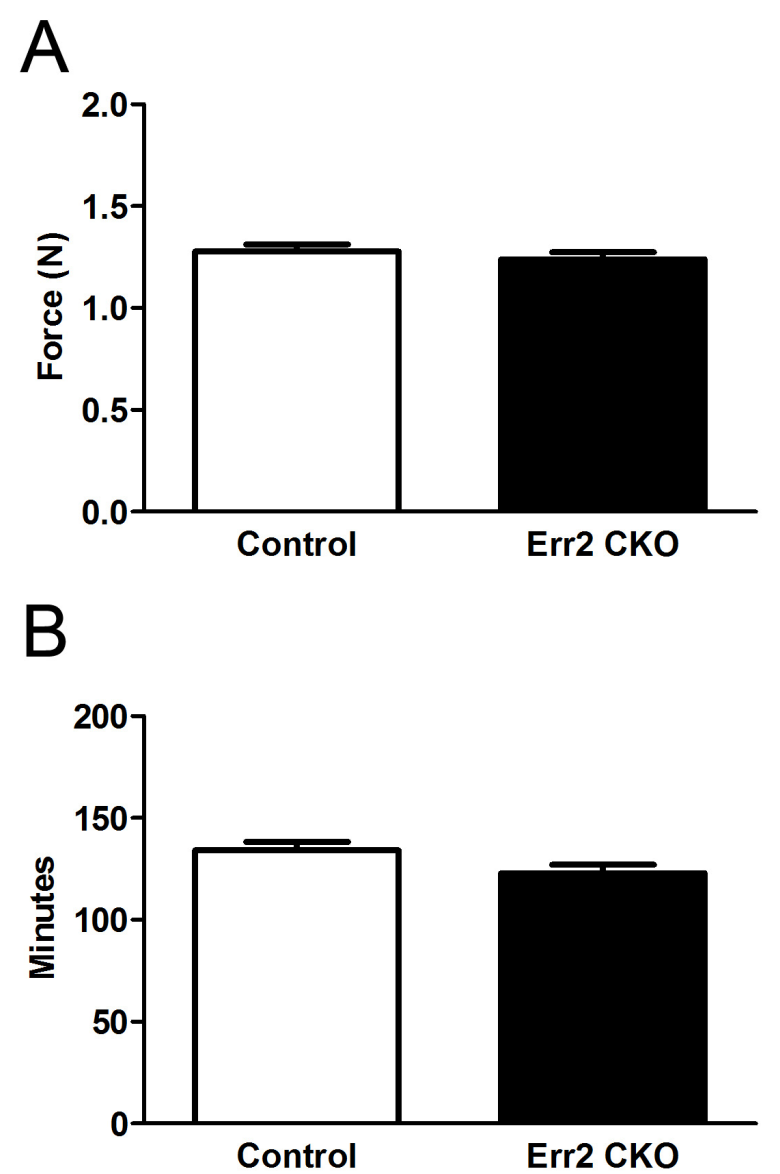

Figure 15. Motor performance in Olig2 ${ }^{\text {Cre }}$; Err2 ${ }^{\text {loxP/loxP }}$ mice. (A) Graph of grip strength in control ( $n=6$, with column) and Err2 mutant mice ( $n=6$, black column) at 7 weeks of age. There was no significant difference in grip strength test. (B) No decline in running performance of the Err2 mutant mice $(n=6)$. The time to exhaustion was measured in 50-day-old mice.

\subsection{The functional role of ERR family in spinal motor neurons}

In the field of developmental biology, chick embryos (Gallus gallus domesticus) are a classical model for studying embryogenesis and neurogenesis, because they are easy to access and manipulate (Figure 16). 
Moreover, the chick is a precocial species which show early neuromuscular maturity and mobility from the time of hatching.

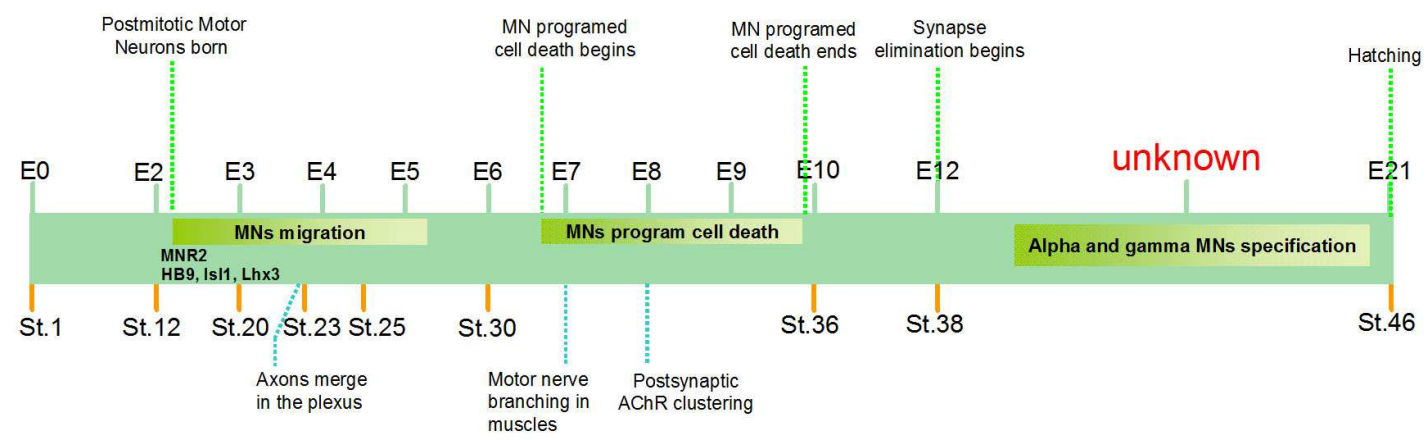

Figure 16. Schematic representation of spinal motor neuron developmental hallmarks in chick embryo. The stages in the schematic timeline correspond to Hamburger-Hamilton stages. Postmitotic motor neurons co-express MNR2, HB9, Isl1, and LIM markers in ventral spinal cord. After E6, motor axons innervate and branch in the target muscles. The formation of acetylcholine receptor clusters begins at E8. The synapse elimination begins at E12. However, the stage of alpha- and gamma-motor neurons specification yet remains to be elucidated.

In order to test whether the Err2 gene has similar functions as the Err3 gene, I chose the chick embryo as an experimental model. First, I performed in situ hybridization to detect the endogenous mRNA expression of both Err2 and Err3 in the developing chick spinal cord. At the same time, Chat expression was detected as a marker for chick lumbar spinal motor neurons. In embryonic chick spinal cord, Chat mRNA expression was detected in all motor neurons in E9 (Figure 17A and 17D) and E15 (Figure 17G and 17J) ventral spinal cord. Both Err2 (Figure 17B and 17E) and Err3 (Figure 17C and 17F) mRNA were strongly expressed in E9 lumbar spinal cord and mainly located in motor neurons in the ventral horn. In addition, in situ hybridization analysis of E15 spinal cord revealed similar Err2 (Figure 17H and 17K) and Err3 (Figure 17I 
and $17 \mathrm{~L}$ ) mRNA expression pattern, suggesting that motor neurons express Err2 and Err3 at similar developing stages.

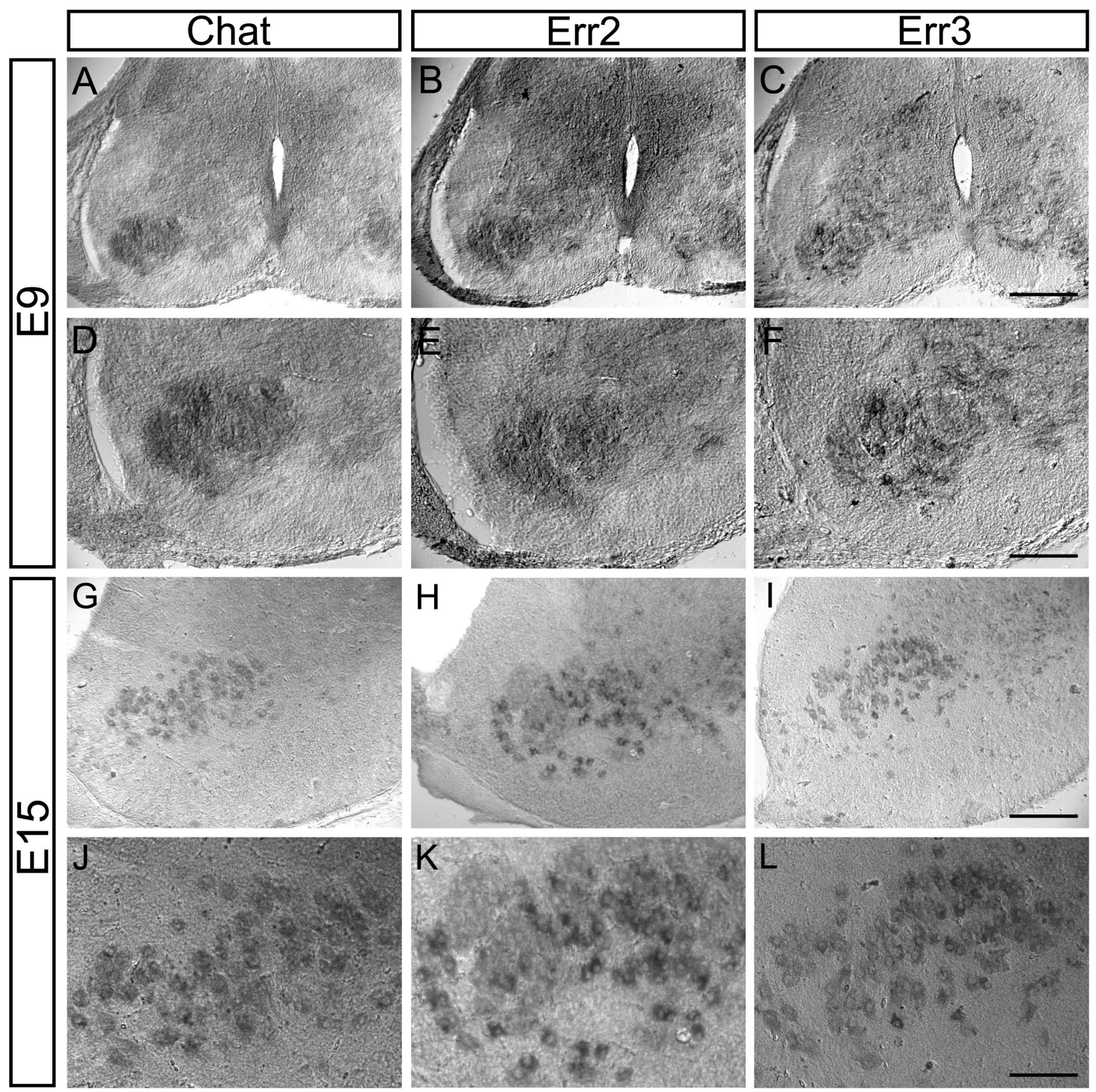

Figure 17. Expression of estrogen-related receptors mRNA in the chick spinal cord. Estrogen-related receptors and Chat mRNAs expression were detected on transversal sections of E9 and E15 chick lumbar spinal cord by in situ hybridization. (A-L) In situ hybridization shows Chat mRNA expression (A, D, G, and J), Err2 mRNA expression (B, E, H, and $\mathrm{K}$ ), and Err3 mRNA expression (C, $\mathrm{F}, \mathrm{I}$, and $\mathrm{L}$ ) in developing chick spinal cord. Both Err2 and Err3 were highly express in chick spinal motor neurons at E9 and E15. Scale bar: (A, B, and C) $200 \mu \mathrm{m}$; (D, E, and F) $100 \mu \mathrm{m} ;(\mathrm{G}, \mathrm{H}$, and I) $200 \mu \mathrm{m}$; (J, K, and L) $100 \mu \mathrm{m}$. 
In ovo electroporation has been used widely as a gene transfer method for chick embryos (Nakamura et al., 2004). This method requires plasmid construction, application of plasmid solution into desired tissue of chick embryo, and application of appropriate electric pulses. I took the advantage of in ovo electroporation technique to study how Err2 or Err3 transcription factors regulate the specification of spinal motor neurons. The Cre-loxP system has used to introduce DNA deletions, insertions, and translocations in specific cells throughout tissues (Sauer, 1987; Luo et al., 2003). In order to study individual gene function in chick motor neurons, Cre recombinase is expressed under the control of postmitotic motor neuron promoter Hb9. After Cre protein removes the loxP-flanked stop signals, ubiquitous promoters can conditionally co-express transgene and fluorescent reporter in spinal motor neurons (Figure 18A). First, Hb9-Cre and loxP constructs were co-electroporated into E2.5 chick neural tube using an electroporator. Motor neurons express the eGFP reporter gene following Cre-mediated recombination. At E9, spinal cords were manually isolated from chick embryos and visualized for eGFP expression. Confocal image analysis of a control experiment demonstrates that nuclear eGFP (NLSeGFP) colocalized with Isl1-positive motor neurons in the ventral spinal cord, suggesting that this Cre-loxP system can be used to specifically overexpress transgene in chick spinal motor neurons (Figure 18B-18D). 
A
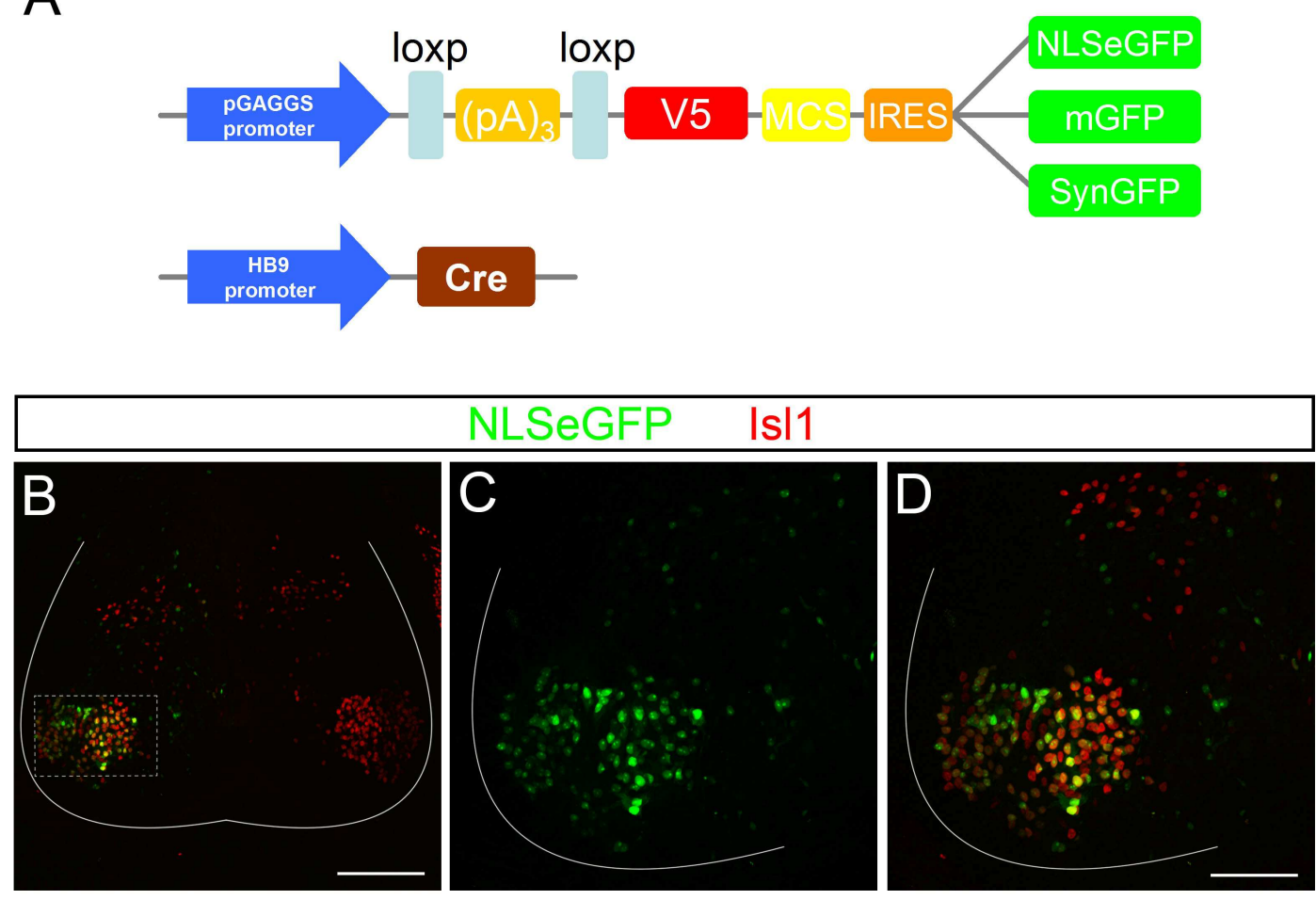

Figure 18. Conditional expression of candidate genes in chick spinal motor neurons using Cre/loxP constructs. (A) Schematic representation of the constructs used to express genes selectively in motor neurons. (B) Confocal images of transversal sections of E9 chick spinal cord showing nuclear eGFP (NLSeGFP) expression in motor neurons. The nuclear eGFP colocalizes with Isl1-positive motor neurons demonstrating the usefulness and specificity of the approach ( $C$ and D, high magnification of boxed area). Scale bar: (B) $200 \mu \mathrm{m}$; (C and D) $100 \mu \mathrm{m}$.

In whole mount chick hindlimb, mGFP can be used as a fluorescent reporter for the visualization of motor axonal innervation in the individual muscles (Figure 19A-19C). Muscle fibers were at the same time marked by anti-myosin heavy-chain protein F59 antibody. The expression of axonally localized mGFP enabled us to map motor axon trajectory in chick hindlimb at E9. Furthermore, in order to trace axon terminals of motor neurons, I used synaptophysin-eGFP fusion construct to label presynaptic motor terminals and postsynaptic acetylcholine receptor clusters were at the same time stained with Alexa Fluor conjugated bungarotoxin (Figure 20B-20D). These results 
demonstrate that Cre-loxP reporter constructs provide excellent tools to facilitate the investigation of gene functions implicated in neuromuscular development.

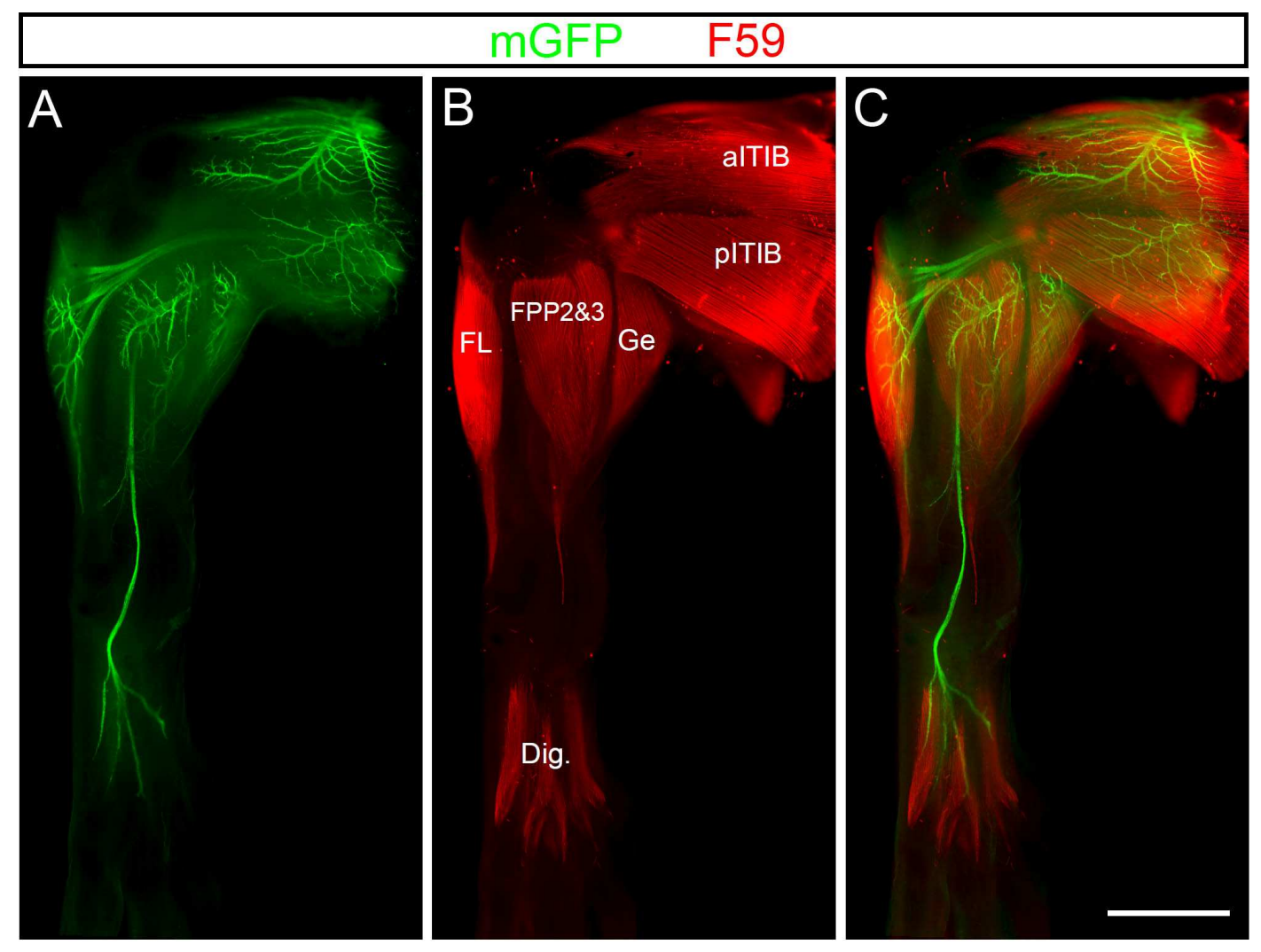

Figure 19. Genetic labeling of spinal motor axon trajectories in chick limbs. (A) Motor axon projections revealed by membrane-targeted mGFP in E9 chick hindlimb. (B) Individual muscles delineated by expression of fast skeletal myosin (F59, red). Motor axons were defined by mGFP immunoreactivity (using anti-GFP antibody) (green). (C) Dorsal view of muscles and motor nerves in the chick hindlimb at E9. alTIB, anterior iliotibialis; PITIB, posterior iliotibialis; $\mathrm{Ge}$, Gastrocnemius externus; FPP2\&3, Flexor perforans et perforatus 2\&3; FL,Fibularis longus; Dig, Digital muscles. Scale bar: $1 \mathrm{~mm}$. 

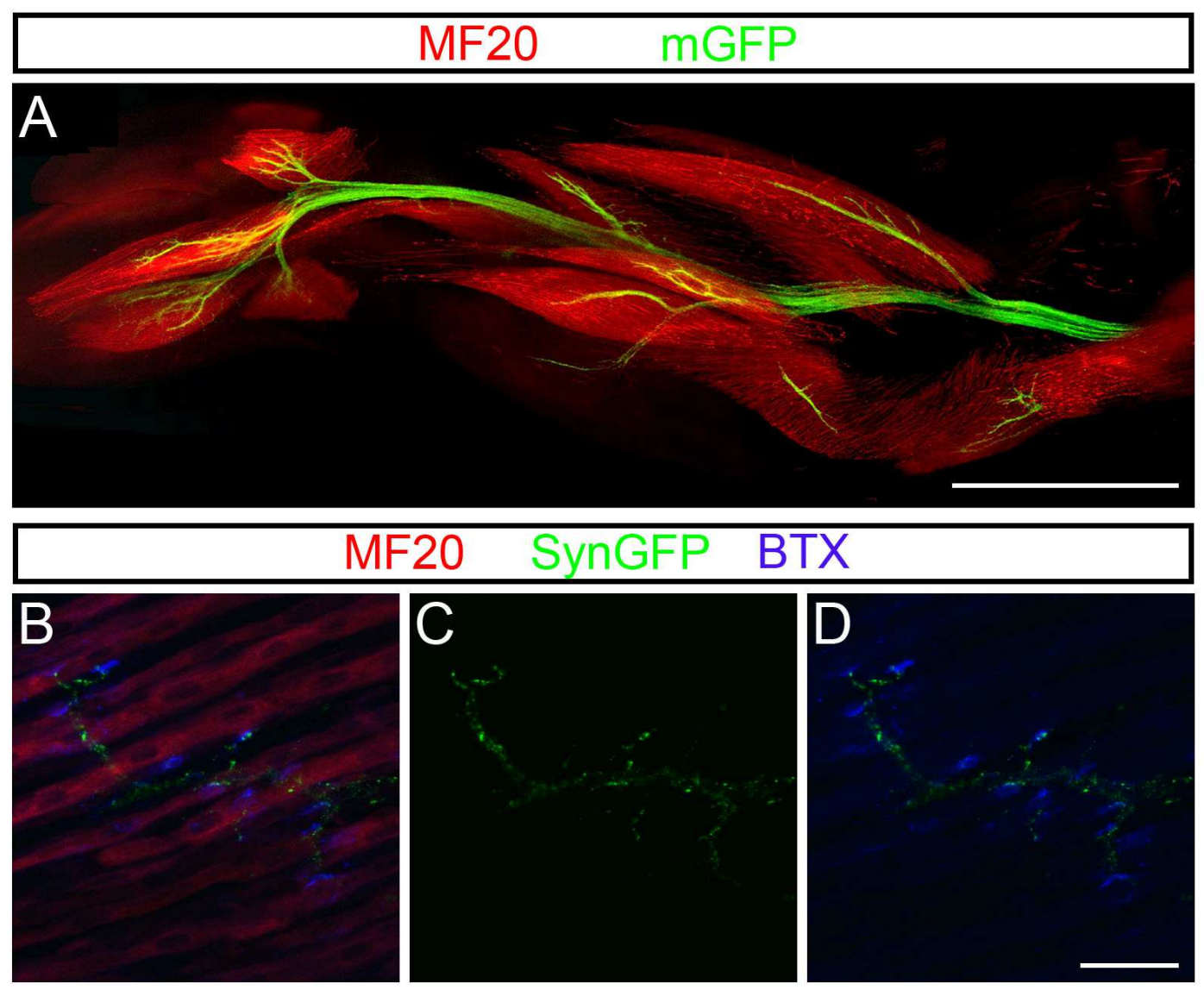

Figure 20. Genetic labeling of presynaptic terminals in motor neurons. (A) Motor axon projections revealed by membrane-targeted mGFP in E9 chick forelimb. (B-D) Neuromuscular junctions are visualized postsynaptically with Alexa Fluor conjugated bungarotoxin (BTX) and presynaptically with synaptophysin-GFP (synGFP). Muscle fibers are visualized by anti-MF20 antibody. Scale bar: (A) $1 \mathrm{~mm}$; (B, C, and D) $30 \mu \mathrm{m}$.

To elucidate the function of estrogen-related receptors in chick motor neurons, I conditionally overexpressed Err2 or Err3 genes in motor neurons at E3. In E9 chick spinal cord, confocal imaging showed that V5 epitope-tagged Err2 (Figure 21A-21C) or Err3 (Figure 21D-21F) specifically colocalized with Isl1- positive motor neurons. 


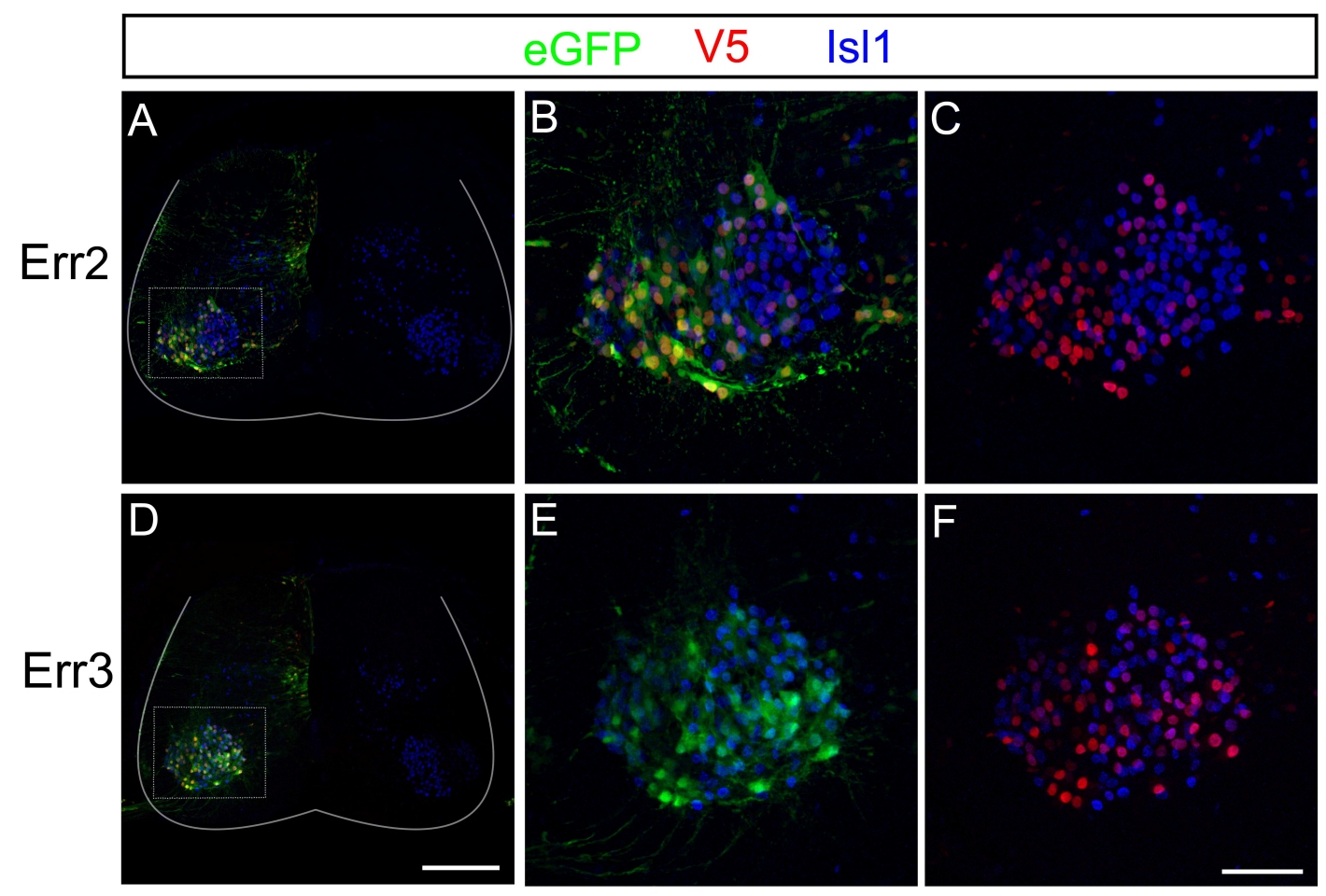

Figure 21. Overexpression of Err2 or Err3 in chick spinal motor neurons by in ovo electroporation of E3 chick spinal cord. A-C shows Err2 expression and D-F shows Err3 expression. Tracer eGFP expression (A, B, D, and E) defines the electroporated side of the spinal cord. Boxed area in $A$ is depicted at higher magnification in $B$ and $C$. Boxed area in $D$ is defined at higher magnification in $\mathrm{E}$ and $\mathrm{F}$. Isl1 expression is shown in blue. Err2 or Err3 expression is shown in red by immunostaining with anti-V5 antibody. Scale bar: (A and D) 200 $\mu \mathrm{m} ;(\mathrm{B}, \mathrm{C}, \mathrm{E}$, and $\mathrm{F}) 30 \mu \mathrm{m}$.

Next, I examined whether ectopic expression of Err2 or Err3 could affect motor projection in the hindlimb. At E9, the expression of mGFP revealed motor axon trajectory and innervation in chick hindlimb (Figure 22A-22D). With this nerve projection map as a guide, I addressed the functional role of Err2 or Err3 in establishing the projection of motor nerves in the developing chick limb. I observed that selective motor projection defects upon Err2 or Err3 overexpression in spinal motor neurons. The nerve branches to the gastrocnemicus muscle were absent in E9 chick hindlimb after Err2 or Err3 overexpression (Figure 22E and 22F). However, further study is needed to 
determine whether this indicates a role for Err2 or Err3 in mediating motor trajectory. In any case, these results indicate that Err2 and Err3 have a similar activity during neuromuscular development.
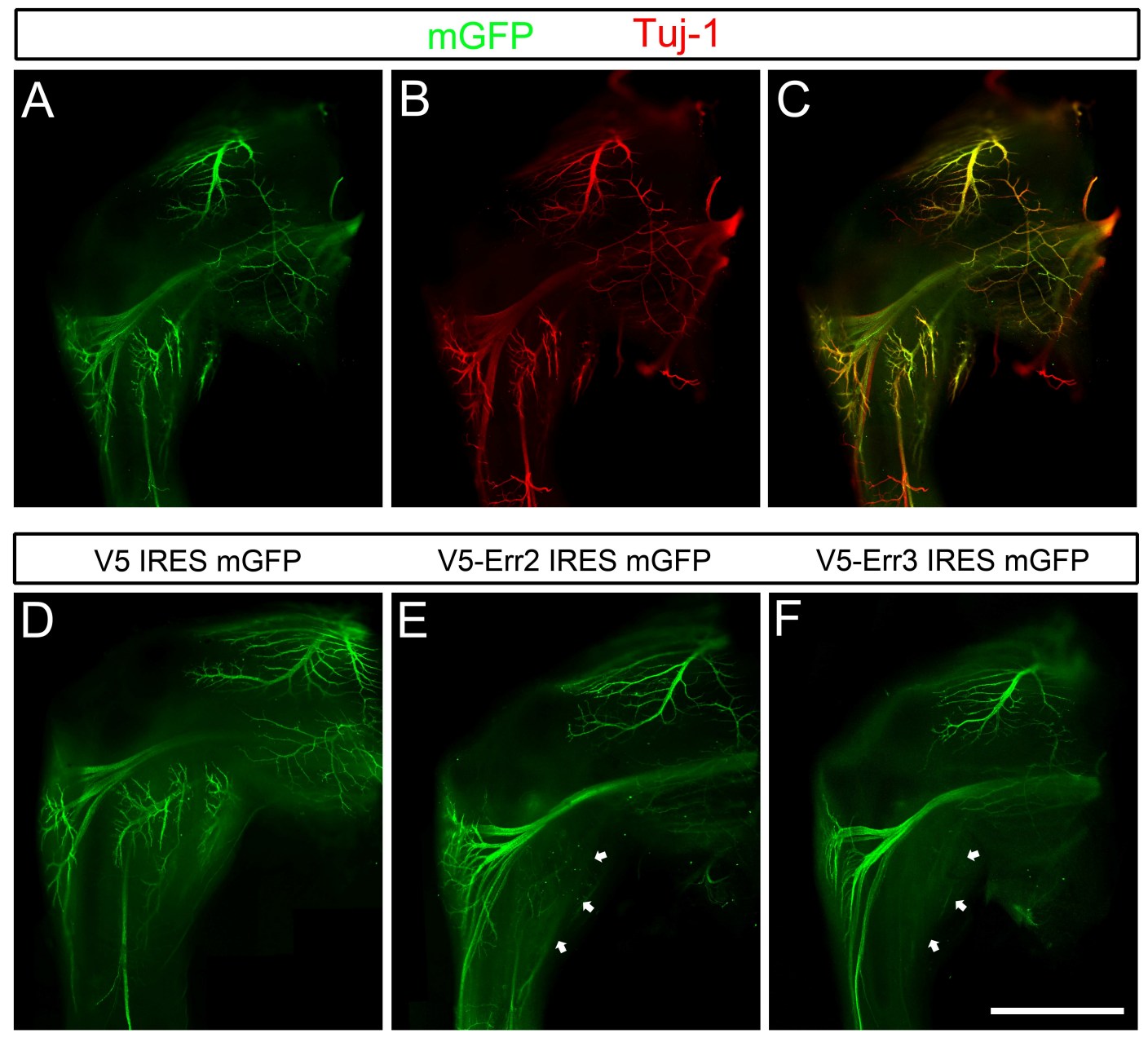

Figure 22. Defects in motor innervation upon Err2 or Err3 overexpression in chick motor neurons. (A-C) Motor nerves were labeled by mGFP (green) and Tuj-1 (red) in E9 chick hindlimbs. Muscle nerves are visualized by mGFP immunoreactivity on whole mount preparations of E9 hindlimbs. (D-F) Comparisons of muscle nerve trajectories from control-mGFP, and forced bicistrinic Err2-mGFP and Err3-mGFP expression in motor neurons in chick embryos at E9 are shown. Motor axon innervation upon forced expression of either Err2 or Err3 is attenuated in gastrocnemicus muscle (arrows). Scale bar: $1 \mathrm{~mm}$. 
The recent characterization of the new markers distinguishing gammaand alpha-motor neurons (Err3, NeuN, Gfra1, and 5Ht1d) provided first insights into the likely time window of gamma-motor neuron specification in postnatal mice (Friese et al., 2009; Enjin et al., 2012). However, the time window of the specification of motor neurons into distinguishable alpha- and gamma-motor neurons remains poorly resolved. I next decided to determine the developmental stages during which morphologically and molecularly distinguishable alpha- and gamma-motor neurons emerge in chick. To achieve this, I combined in ovo retrograde tracing with immunofluorescence. The CTB was injected into chick hindlimb musculature. After 48 hours, retrogradely labeled motor neurons could be visualized in the spinal cord. By using this approach, I found that a subset of motor neurons consistently display either complete absence or lower expression of NeuN at E18 (Figure 23A-23C).

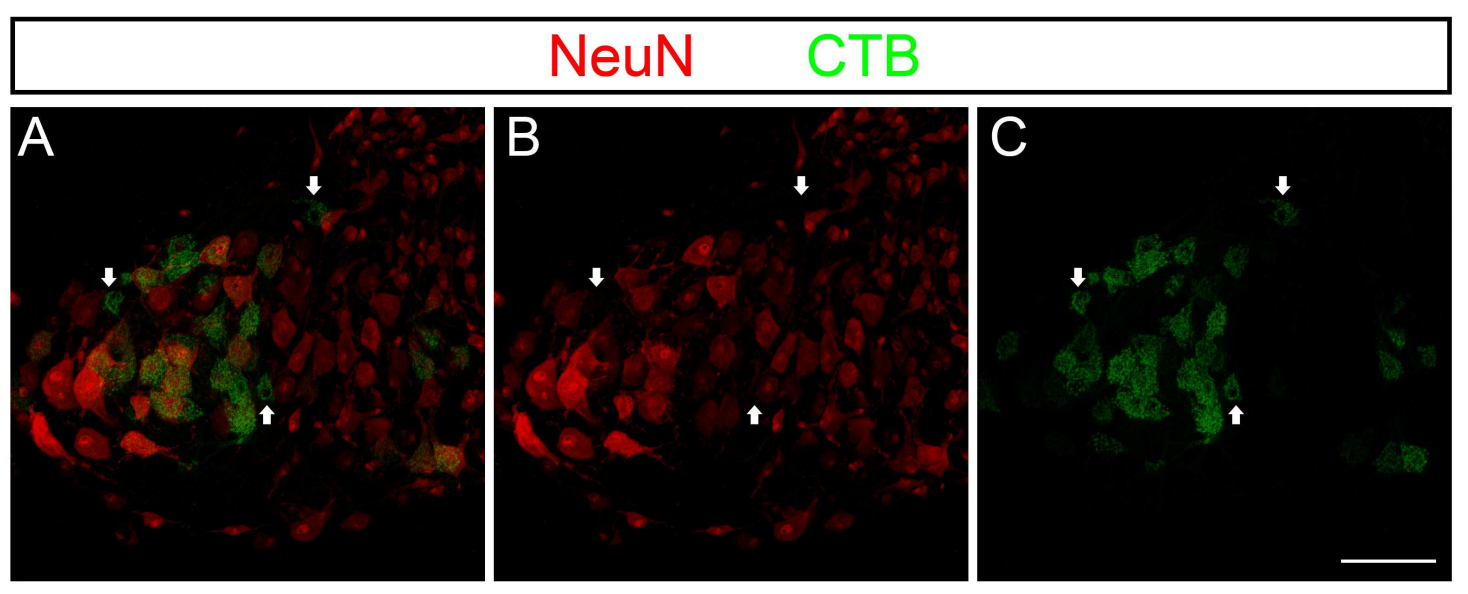

Figure 23. Putative gamma-motor neurons displaying low NeuN expression in E18 chick spinal cord. (A) Immunohistochemical analysis of NeuN (red) and CTB (green) expression in chick lumbar motor neurons. Spinal motor neurons were labeled retrogradely by in ovo intramuscular injection of CTB into iliotibialis muscle at E16. (B-C) Arrows indicate small CTB-positive putative gamma-motor neurons lacking NeuN expression in E18 chick spinal cord. Scale bar: $100 \mu \mathrm{m}$. 
It is concretely established by several studies that the soma area of gamma-motor neurons are consistently smaller than those of alpha-motor neurons (Friese et al., 2009; Shneider et al., 2009; Manuel and Zytnicki, 2011). Therefore, investigation of the soma size distribution in chick lumbar spinal cord would enable us to gain an insight in to the time point at which gamma-motor neurons appear during embryogenesis. To this end, I have performed soma size quantification studies on retrogradely traced chick motor neurons post intramuscular CTB injection into the hindlimb. The results indicate that there is an appearance of small-sized motor neuron population at E18 and E20 in lumbar spinal cord, but not at E15 (Figure 24A-24C). Further, as it is known that small-diameter gamma-motor neurons are characterized by low expression of NeuN, I have correlated the sizes of CTB labeled motor neurons with the expression of NeuN antigen. The analysis of NeuN intensity in retrogradely traced motor neurons demonstrates that the small-diameter neurons have a lower NeuN expression and therefore are gamma-motor neurons (Figure 24D). These data suggest that small-diameter gamma-motor neurons marked by low NeuN expression appear at E18 in embryonic chick motor neurons. 

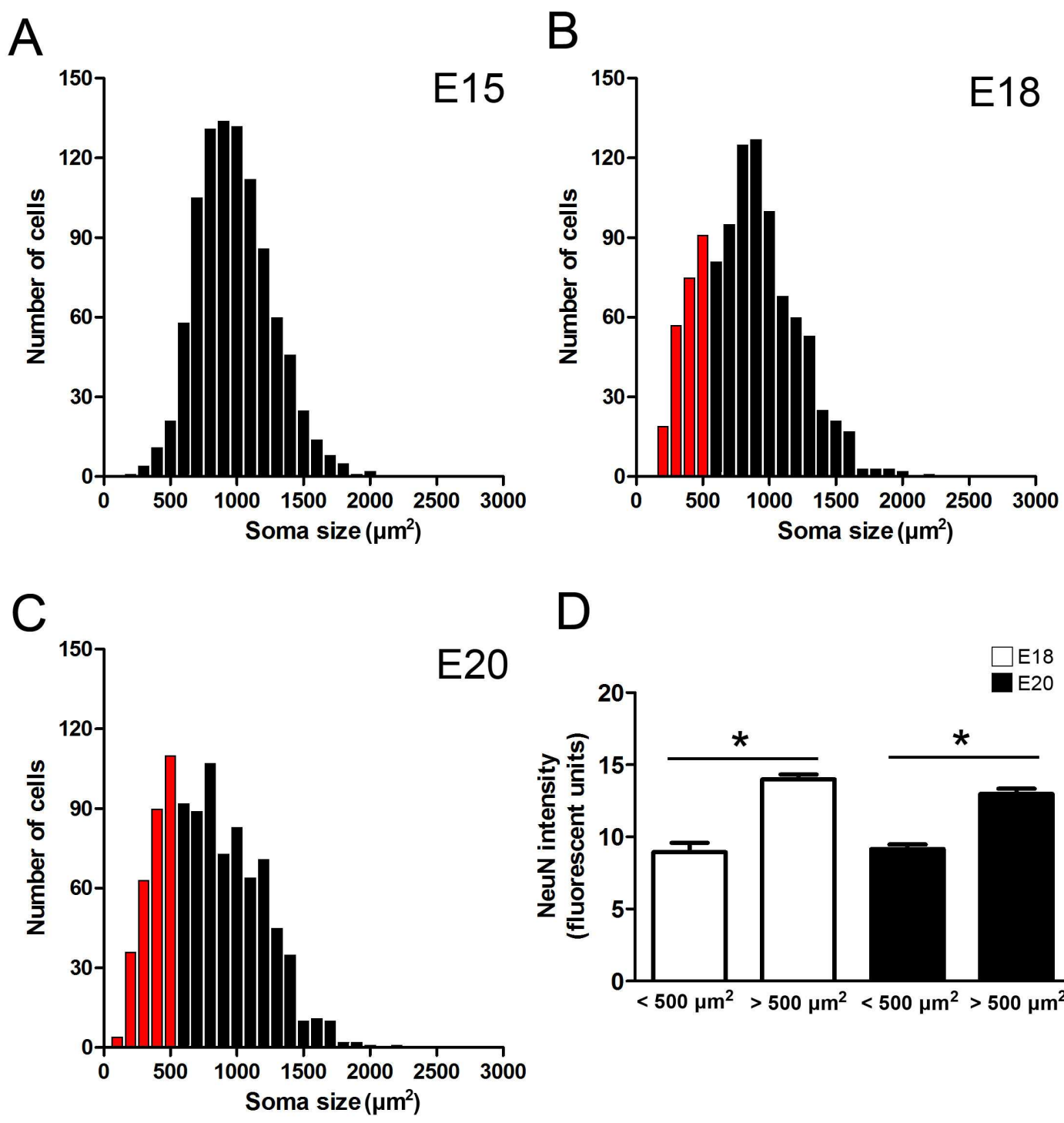

D

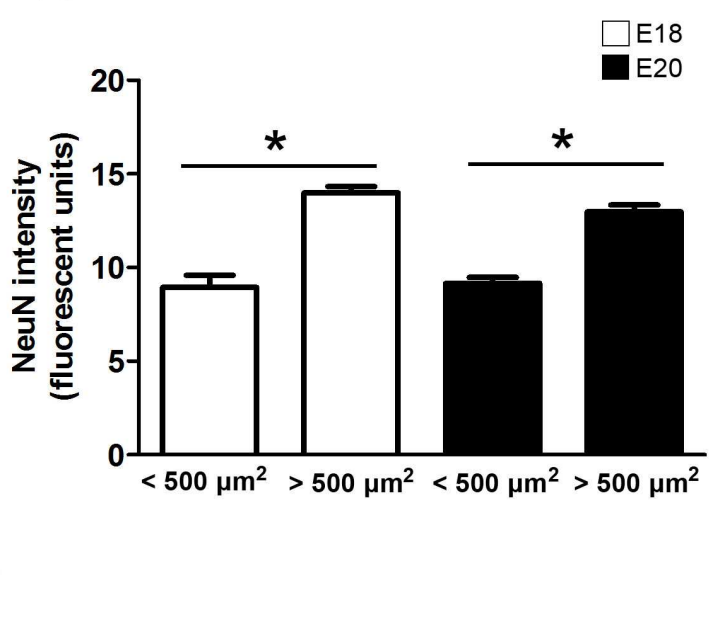

Figure 24. Motor neuronal soma size distribution in developing chick lumbar spinal cord. (A) Size distribution of retrogradely traced motor neurons at E15. (B and C) Small-sized motor neuronal population ( $<500 \mu \mathrm{m}^{2}$, red columns) appears at E18 and E20. (D) Quantitative analysis of NeuN antigen expression in motor neurons at E18 and E20. Small motor neurons $\left(<500 \mu \mathrm{m}^{2}\right.$, red columns) have a lower NeuN expression than lager motor neurons ( $>500$ $\left.\mu m^{2}\right)$. ( $n=955$ in $E 15, n=1026$ in $E 18$, and $n=1000$ in E20. Asterisk denotes significant difference (Student's $t$-test) * $p<0.05$. 


\subsection{Stable transgene expression in late gestation chick spinal cord}

In order to address whether Err2 or Err3 activity could drive gamma-motor neuron subtype specification, I decided to overexpress Err2 or Err3 in motor neurons of the late-gestation chick embryo. However, conventional plasmids only maintain the expression of transgenes for several days because these constructs are not able to integrate into the host genome. To overcome this problem, I used a transposon-mediated gene transfer method based on the Medaka fish Tol2 transposon system to achieve long-term transgene expression in the chick embryo upon in ovo electroporation. Transposable genetic elements have been reported as powerful gene transfer tools in plant and invertebrate models. Recently, methods based on vertebrate transposable elements have become available that allow stable somatic transgenesis in species including zebrafish, Medaka, chick, and mice (Kawakami, 2007; Suster et al., 2009). Basically, these methods are based on a transposase that recognizes a pair of core transposon sites flanking the transgene expression cassette, resulting in the recombination of the transgenes sequences from the vector and its subsequent integration into the host genome. I used a newly developed system that combines the Tol2 transposon core elements combined with a powerful $C M V$ enhancer to stably express transgenes in chick motor neurons throughout gestation (Figure 25A). Using this system, I could routinely achieve strong eGFP expression in the late gestation chick spinal cord (Figure 25B-25C). Confocal images taken from the E15 lumbar spinal cord after injection of fluorescent CTB into the chick hindlimb. Interestingly, these experiments showed that the stable eGFP transgene expression was preferentially restricted to retrogradely labeled motor neurons (Figure 
25D-25E), suggesting that this transposon-based vector system could be used to restrict transgene expression in chick motor neurons during late stages of gestation (Figure 25).
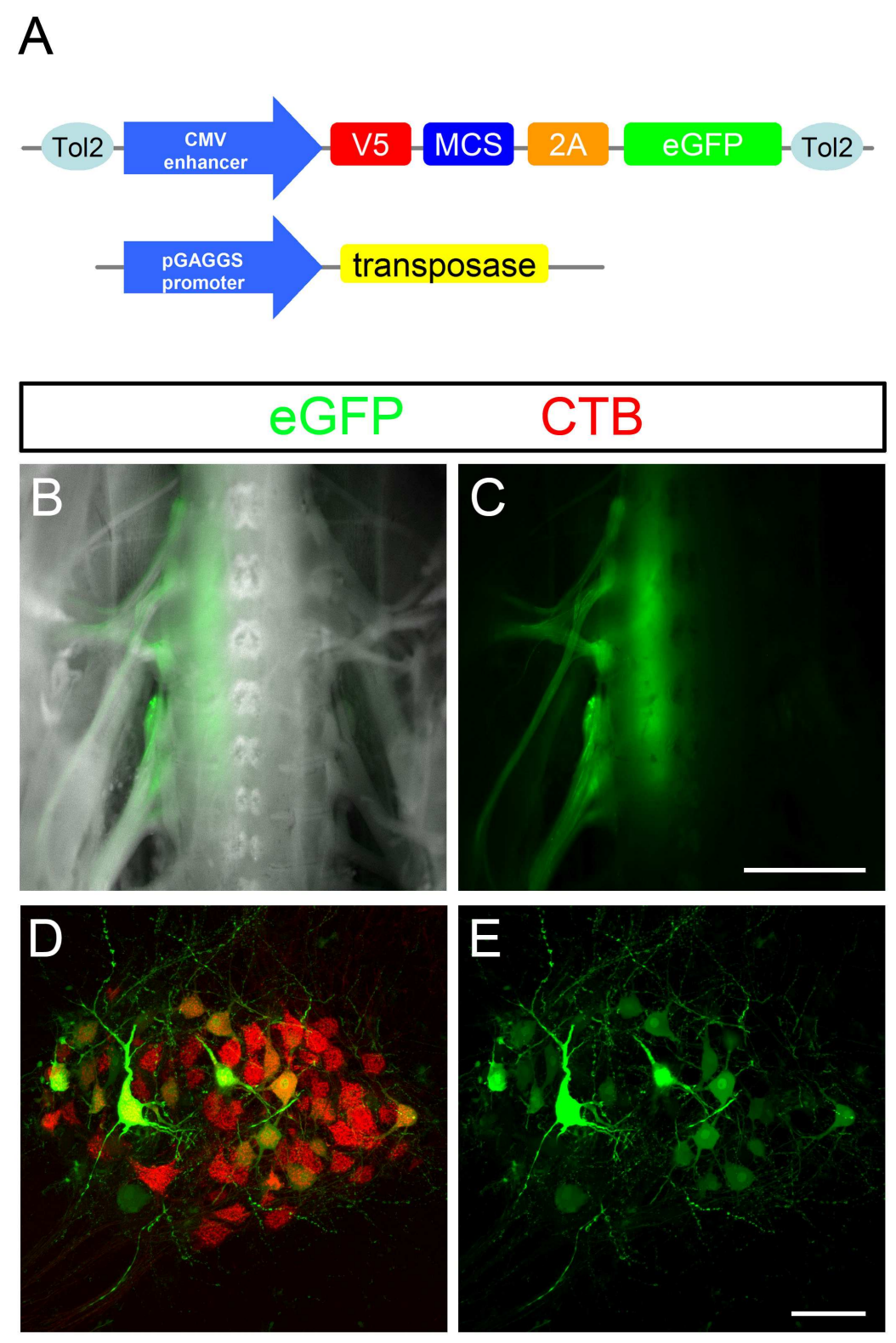

Figure 25. Stable transgene expression in late-gestation chick spinal cord. (A) Schematic representation of the Tol2 constructs used in the present study. (B-C) The ventral view of the E15 spinal cord showing unilateral eGFP expression. (E) CMV enhancer driven eGFP and chick $\beta$-actin promoter driven transposase mediate specific and stable eGFP expression in motor neurons). (D) Colocalization of eGFP and CTB demonstrates that CMV enhancer drives transgene expression specifically in motor neurons. Scale bar: (B and $C) 3$ $\mathrm{mm}$; (D and E) $100 \mu \mathrm{m}$. 
As a basis for testing impacts of forcedly expressing Err2 or Err3 activity in motor neurons, I next examined in detail the emergence of morphological and molecular hallmarks of gamma-motor neurons during chick gestation. I found that NeuN expression becomes extinguished in a subset of small-diameter motor neurons by E18. Next, I electroporated the E3 neural tube with transposon vectors to forcibly express V5 epitope-tagged Err2 or Err3, followed by examination at E15. Upon control vector transfection (V5-expression cassette without insert), most eGFP-labeled spinal motor neurons coexpressed NeuN antigen (Figure 26A-26C). At the same time, eGFP-positive motor neurons expressing V5-Err2 (Figure 26D-26F) or V5-Err3 (Figure 26G-26I) either showed lower or no expression of NeuN. Furthermore, to investigate the mechanisms by which Err2 or Err3 regulate motor neuron specification, I fused Err2 or Err3 protein with the VP16 transcriptional activation domain (VP16) or the engrailed repressor domain (EnR). Expression of VP16:Err2 (Figure 26J-26L) or VP16:Err3 (Figure 26M-260) dramatically lowered or extinguished NeuN expression in motor neurons. Furthermore, quantification of the soma diameter distribution revealed that there was a slight increase in the population of small-sized motor neurons $\left(<500 \mu \mathrm{m}^{2}\right)$ upon V5-Err2 (Figure 27B) or V5-Err3 expression (Figure 27C). Notably, expression of VP16:Err2 (Figure 27D) or VP16:Err3 (Figure 27E) resulted in sharply increased numbers of small-diameter motor neurons. In contrast, expression of EnR:Err2 (Figure 27F) or EnR:Err3 (Figure 27G) did not alter the soma diameter distribution compared to the control vector (Figure 27A). 


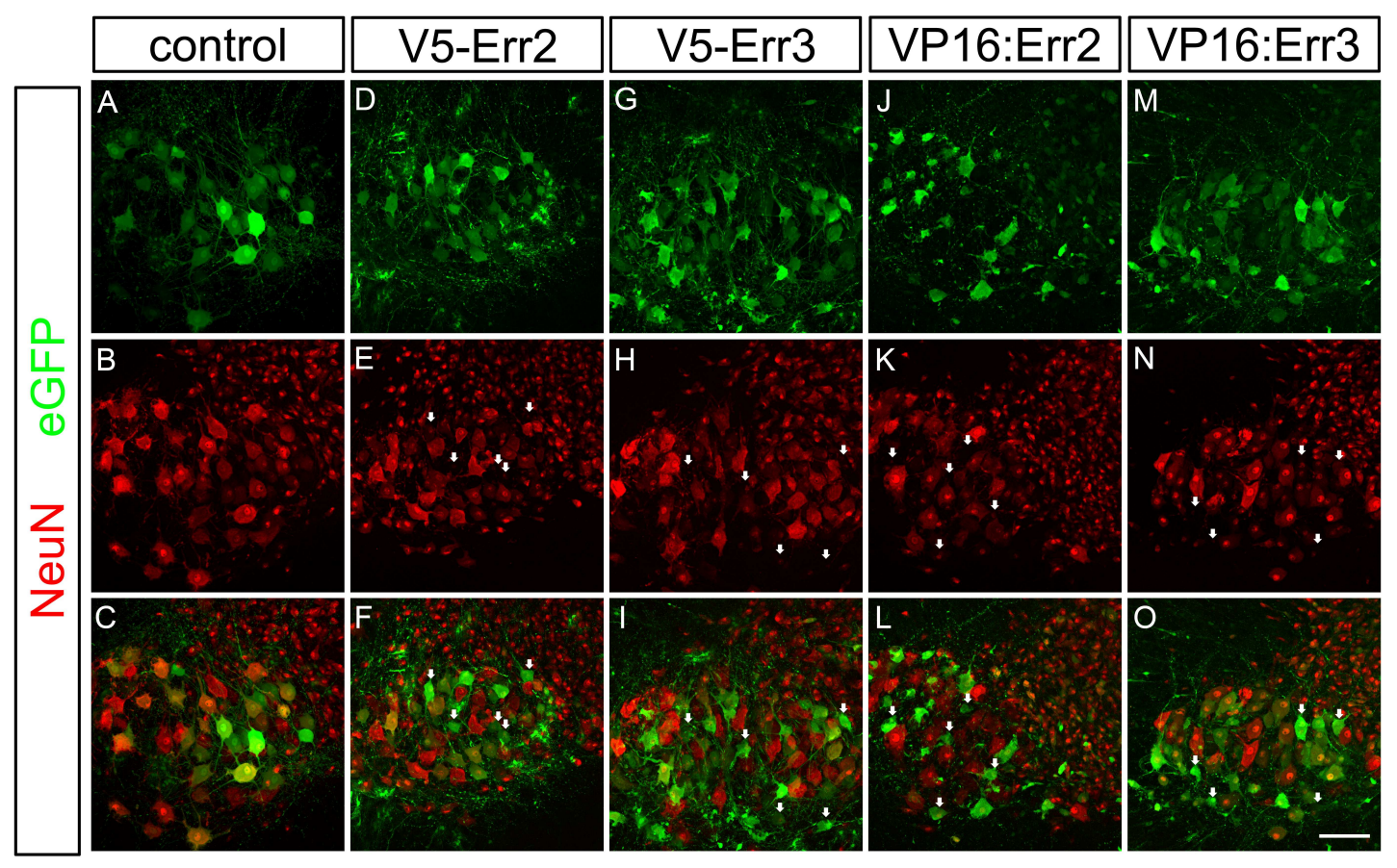

Figure 26. Overexpression of Err2 or Err3 during late-gestation chick embryo resulted in an increased numbers of gamma-motor neurons. In (A-C) control group, (D-F) V5-Err2, (G-I) V5-Err3, (J-L) VP16:Err2, and (M-O) VP16:Err3, eGFP defines the cell boundary of spinal motor neurons and arrows indicate small-diameter gamma-motor neurons displaying low NeuN expression (red). Scale bar: $100 \mu \mathrm{m}$.

In addition to soma size, gamma-motor neurons have slower axonal conduction velocities and smaller axon caliber compared to alpha-motor neurons (Kanning et al., 2010; Manuel and Zytnicki, 2011). Upon forced expression of VP16:Err2 or VP16:Err3, ventral root cross-sections revealed a marked decrease in axon caliber (Figure 28). Taken together, these data suggest that Err2 and Err3 act as transcriptional activators to promote the specification of gamma-motor neurons in a subset of motor neurons. The results of my thesis project thereby provide first insights into the molecular determinants that drive the diversification of motor neurons into functionally specialized subtypes. 

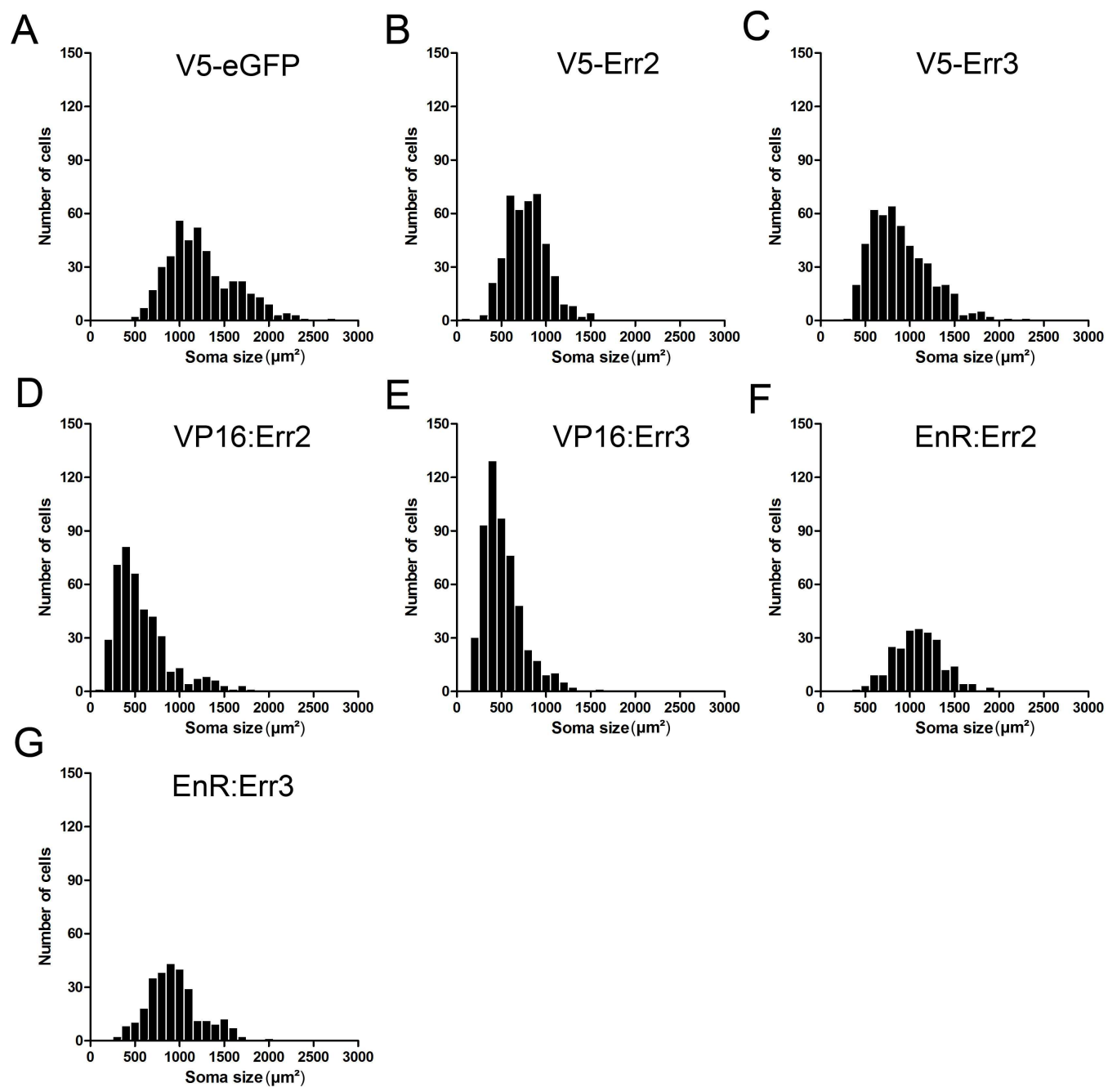

Figure 27. Motor neuron soma size distribution upon Err2 or Err3 overexpression. (B) V5-Err2 and (C) V5-Err3 group have a slight increase in small-diameter motor neurons $(<500$ $\mu \mathrm{m}^{2}$ ) when compared to controls (A). Upon overexpression of (D) VP16:Err2 or (E) VP16:Err3 construct, an increase in number small-sized motor neuron population in the spinal cord was observed. However, (F) EnR:Err2 and (G) EnR:Err3 group have no difference in soma size distribution ( $n=420$ in V5-eGFP, $n=421$ in V5-Err2, $n=481$ in V5-Err3, $n=424$ in VP16:Err2, $n=540$ in VP16:Err3, $n=238$ in EnR:Err2, and $n=276$ in EnR:Err3). 

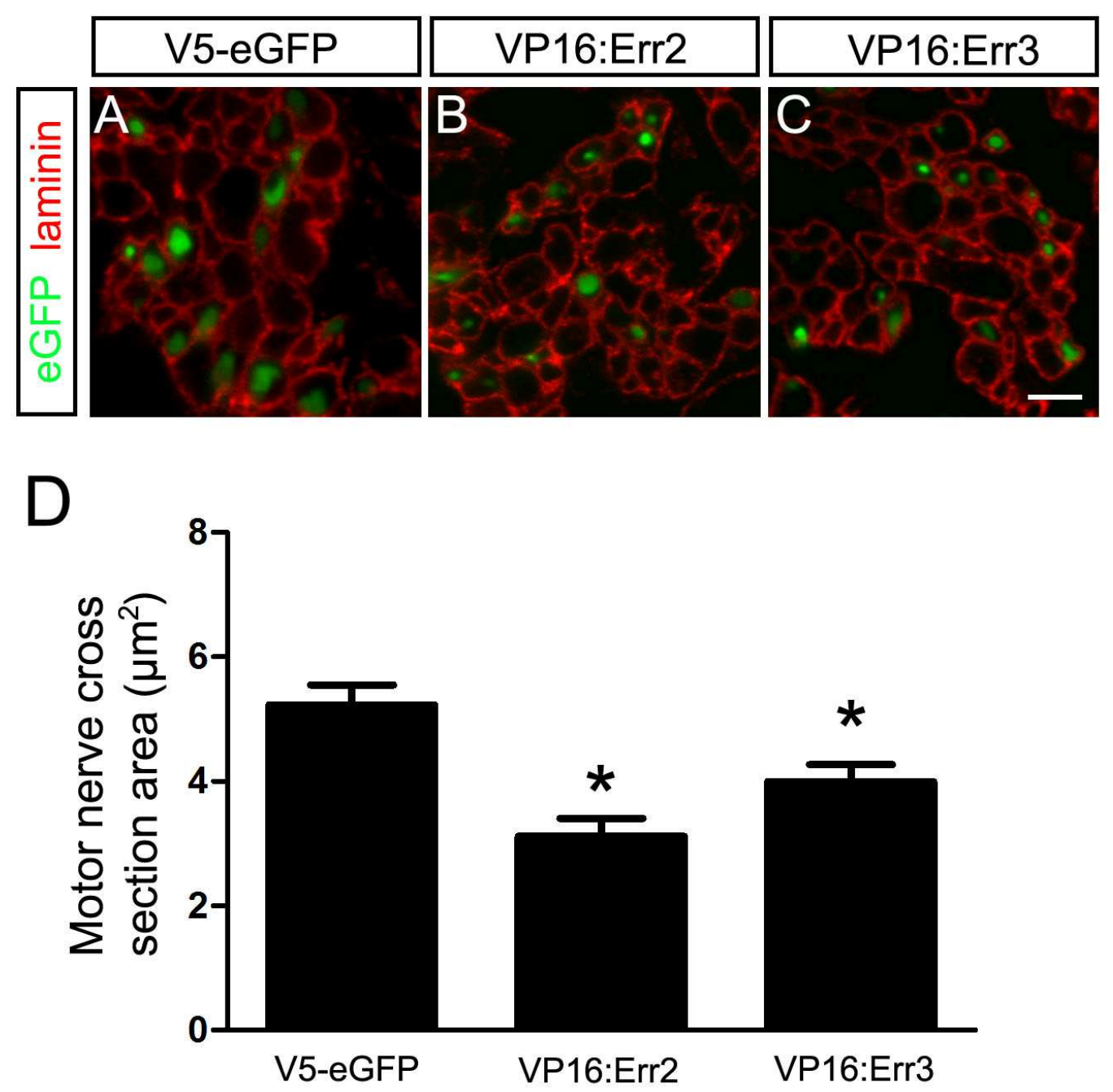

Figure 28. Overexpression of VP16:Err2 or VP16:Err3 in chick motor neurons reduces axonal diameter. (A-C) Confocal image of a chick motor nerve cross section revealed by laminin (red). (D) Quantification of motor axon diameter upon VP16:Err2 or VP16:Err3 overexpression. The axon diameter of VP16:Err2 or VP16:Err3 overexpressing motor neurons were reduced when compared to the control group. ( $n=154$ in V5-eGFP, $n=126$ in VP16:Err2, and $n=149$ in VP16:Err3. Asterisk denotes significant difference (Student's $t$-test) ${ }^{*} p<0.05$. Scale bar: $5 \mu \mathrm{m}$. 


\section{Discussion}

Proper assembly of the neuromuscular circuitry is indispensable for locomotion. The development of this circuitry depends on the orchestrated assembly and specification of several neuronal components (alpha-fast and alpha-slow motor neurons, gamma-motor neurons, and premotor interneurons) and the muscle fiber subtypes (type I, Ila, IIx, and Illb) (Kanning et al., 2010). Many transcription factors have been identified that confer motor neuronal identity. For example, transcriptional cascades involving Hox and LIM homeodomain proteins lead to the formation of distinct motor columns and motor pools in the developing spinal cord (Jessell, 2000; Shirasaki and Pfaff, 2002).

The functional diversification of motor neurons within a motor pool is fundamental for smooth and graded movements (Kandel et al., 2000). On a broad level spinal motor neurons can be subdivided in to alpha- and gamma-motor neurons. Alpha- motor neurons are further classified into fast, intermediate, and slow motor neurons which control the contraction of respective muscle fibers (Kanning et al., 2010). Despite being generated from the same lineage of the progenitor motor neuronal domain, how the functional diversification into alpha- versus gamma-motor neurons as well as slow versus fast motor neurons arises remains elusive (Brownstone and Stuart, 2011). Recently, first genetic markers have been proposed to selectively label fast (Chodl and Calca) or slow motor neurons (SV2a) (Chakkalakal et al., 2010; Enjin et al., 2010). However, so far none of the studies have identified mechanisms or factors that are involved in the specification of fast or slow motor neuronal phenotypes. While alpha-motor neurons control the 
contraction of extrafusal muscle fibers, the small-diameter gamma-motor neurons regulate the sensitivity of muscle spindles by controlling the contraction status of intrafusal muscle fibers. The gamma-motor neurons thereby adjust the tone of skeletal muscles through spindle afferent feedback that controls alpha-motor neuronal activity. They are present at all levels of the spinal cord intermingled with the more numerous alpha-motor neurons. Gamma-motor neurons can be distinguished from alpha-motor neurons by the absence of direct la synaptic input, small soma size, axon diameter and also by the absence of C-type boutons (Kanning et al., 2010). Several markers for gamma-motor neurons which include Err3, Gfra1, and 5HT1D have been reported (Friese et al., 2009; Shneider et al., 2009; Enjin et al., 2012). As is the case with alpha-motor neurons, it is not known whether these markers contribute to the specification and cell type identity of gamma-motor neurons. Therefore, a clear understanding of the molecules and pathways involved in acquisition of gamma-motor neuronal identity is missing. Using a combination of experimental tools, I have identified the orphan nuclear receptor Err2 as a cell fate determinant for gamma-motor neurons, which seems to act in a redundant manner with the closely related Err3 protein.

My initial studies were aimed towards identifying genes differentially expressed in functional motor neurons subtypes. To this end, I have performed microarray analysis on retrogradely identified motor pools predicted to be enriched in different motor neurons subtypes in E18.5 mice. This study has revealed that several transcriptional factors and ion channels are differentially expressed in these motor neuron pools. Err2 was one of the transcription factors that is highly enriched in the neurons of the predominantly "fast" rectus femoris motor pool (Figure 9F). ERR proteins are orphan nuclear receptors 
and have been implicated in various pathways regulating cell metabolism and mitochondrial biogenesis. The ERR family proteins has three members, Err1, Err2, and Err3. Further, expression analysis using in situ hybridization and immunostaining has revealed that Err2 is expressed by a subpopulation of small-diameter motor neurons. This restricted expression in a small-diameter motor neurons population is found from early postnatal stages onwards (Figure 13 and 14). A recent report has shown that the ERR family member, Err3 is similarly expressed in small-diameter motor neurons and the same study suggested that gamma-motor neurons can be distinguished from alpha-motor neurons by high expression of Err3 and low or no expression of NeuN (Friese et al., 2009). This indicates that gamma-motor neurons display low expression of NeuN compared to alpha-motor neurons. Another recent study also found that NeuN expression is higher in alpha-motor neurons compared to gamma-motor neurons (Misawa et al., 2012). The mechanism mediating the down-regulation of NeuN antigen in gamma-motor neurons is not well characterized. So far, two isoforms of NeuN protein (46- and 48-kDa) have been identified (Lind et al., 2005). The $48-\mathrm{kDa} \mathrm{NeuN}$ isoform is predominantly expressed in the cytoplasm whereas the smaller 46-KDa isoform is predominant in the nucleus. My observations show that both NeuN isoforms can be detected in the spinal motor neurons (Friese et al., 2009; Shneider et al., 2009). It is noteworthy to mention that in the nucleus, NeuN is a phosphoprotein which is found in the low chromatin density area, but not in the densely packed DNA, indicating that a NeuN-associated epigenetic program may be involved in a direct or indirect fashion to regulate the differentiation and specification of gamma-motor neurons (Hsieh and Gage, 2005). 
My results related to the expression of Err2 (Figure 13 and 14) show a trend which parallels that of the expression pattern of Err3, in that both being present in small-diameter motor neurons which express low or no expression of NeuN. Further, I found that overexpression of either Err2 or Err3 in the embryonic chick spinal cord produced similar peripheral innervation defects. Together, this has lead me to hypothesize that Err2 and Err3 could act as redundant determinants of gamma-motor neuron identity.

To validate my hypothesis, I have first systematically tested the expression of Err2 in the mouse spinal cord. The results indicated that Err2 is expressed in small-diameter motor neurons characterized by low NeuN expression (revealed by triple labeling with anti-vAChT, anti-NeuN, and anti-Err2 antibodies) at all rostrocaudal levels. Based on these observations, I concluded that Err2 is selectively expressed by gamma-motor neurons. The discrepancy of Err2 being highly enriched in the rectus femoris (fast motor pool) motor neurons may be due to a regional specialization or due to the temporally separated development of extensors versus flexor motor neurons.

Then, to address the impact of Err2 on motor neuronal identity in vivo, I have used the chick embryo system. First, I have examined whether Err2 and Err3 are expressed in chick motor neurons. To test this issue, I have performed in situ hybridization analysis on the embryonic chick spinal cords at various developmental stages. The results indicate that Err2 and Err3 are initially expressed by all motor neurons, and then become increasingly restricted to small-diameter motor neurons at late stages (E18) similar to the expression of Err2 and Err3 in mouse motor neurons. Further, I found that gamma-motor neurons seem to appear in embryonic chick spinal cords at late developmental stages (E18) by performing immunohistochemistry (with 
anti-NeuN antibody) on retrogradely traced motor neurons. This late appearance/specification of gamma-motor neurons in chick embryo presented me with the challenge of maintaining transgene expression using established in ovo electroporation techniques. At these late stages, transient transfection of expression constructs by in ovo electroporation were not detected anymore. To overcome this hurdle, my colleagues and I have designed a Tol2 transposon system to selectively and stably express a gene of interest in the chick motor neurons. eGFP from control constructs cloned into this system show strong expression during the entire in ovo gestation (and beyond) in chick (Figure 25). This provides a powerful tool which enabled me to stably overexpress candidate genes in embryonic chick spinal cord.

Then, I asked whether overexpression of Err2 can confer gamma-motor neuron properties in embryonic chick motor neurons. To address this question, I overexpressed Err2 in motor neurons using the Tol2 transposon system in ovo. The results clearly demonstrate that upon forced expression of Err2 in motor neurons, there is a decrease in both soma size and NeuN expression (Figure 26 and 27) - a feature shared by gamma-motor neurons in both mouse and chick. In addition, cross sections of the ventral roots revealed that Err2 overexpression results in smaller axon calibers (Figure 28). It is already known that gamma-motor neurons have smaller caliber axons compared to alpha-motor neurons. The same impact on motor neurons was obtained upon forced expression of Err3 (Figure 26). Thus, Err2 and Err3 both appear to promote the appearance of hallmarks of gamma-motor neurons, while suppressing characteristics of alpha-motor neurons.

A study published in 2010 has proposed Err2 to be a candidate marker for slow motor neurons, mainly based on the small-diameter of 
Err2-expressing motor neurons in mice (Enjin et al., 2012). The present study refutes this proposal at multiple levels. First, the higher levels of Err2 expression in the mainly predominantly "fast" rectus femoris motor neuron pool, compared to the predominantly "slow" soleus motor neuron pool is inconsistent with an expression in slow motor neurons. Second, Err2 is expressed in NeuN-negative motor neurons. Since slow motor neurons are alpha-motor neurons and would therefore be expected to express high levels of NeuN (Misawa et al., 2012Misawa et al., 2012), this again is not consistent with an expression in slow motor neurons. Third, Err2-positive motor neurons overlap with the population of smallest diameter motor neurons in the overall bimodal size distribution of motor neurons, which again indicates an association with gamma-motor neurons, but not alpha-motor neuron subtypes. Fourth, Err2 shows overlapping distribution with the related Err3, which is an established gamma-motor neuron marker. Fifth, my forced expression of both Err2 and Err3 promote gamma-motor neuron characteristics, but not slow (alpha) motor neuron characteristics. Taken together, based on these numerous observations, I concluded that Err2 is a specific marker for gamma-motor neurons and not for slow motor neurons.

ERR proteins are thought to act as transcriptional regulators. I therefore asked whether the promotion of gamma-motor neuron characteristics by Err2 and Err3 depends on transcriptional activation or repression. I tested this by fusing the VP16 transcriptional activation domain to Err2 and Err3. The results indicate that chimeric VP16:Err2 and VP16:Err3 proteins very efficiently promoted the premature acquisition of gamma motor neuronal characteristics, such as small soma size and low levels of NeuN (Figure 27). Fusion of Err2 to the potent engrailed transcriptional repressor $(E n R)$ domain showed that the 
resulting chimeric EnR:Err2 protein lacked the ability to induce gamma-motor neuronal characteristics (Figure 27). This suggested that Err2 and Err3 normally act as transcriptional activators to promote gamma-motor neuron specification. Preliminary data generated in collaboration with another PhD student in the group indicate that overexpression of both Err2 and Err3 produce similar changes in the electrophysiological properties of motor neurons (work in progress). Taken together, based on these results, I propose that Err2 and Err3 act as transcriptional activators to promote gamma-motor neuronal identity.

To my knowledge, this is the first identification of a cell fate determinant of functional motor neuronal subtype. Since Err2 is able to promote the acquisition of gamma-motor neuron fate, I asked whether Err2 would also be required for gamma-motor neuron specification. However, conditional knockout mice in which Err2 is selectively inactivated in the motor neuron lineage did not reveal a defect in gamma-motor neuron specification, based on immunohistochemical analysis of motor neuronal diameter and NeuN expression. Further, the Err2-deficient mice showed motor behaviors that were indistinguishable from the control group. The likely explanation for the absence of a detectable phenotype in the mutant mice is that Err2 and Err3 act in a redundant manner to promote gamma-motor neuronal fate acquisition. This is consistent with the identical phenotypes in motor neuron fate and projection patterns induced by forced Err2 and Err3 expression in chick. Taken together, these findings indicate that Err2 and Err3 act in redundant manner to confer gamma-motor neuron fate in a subset of spinal motor neurons. The question whether both proteins would indeed be required for the specification of gamma-motor neurons awaits further studies, however, such as by 
simultaneous conditional inactivation of Err2 and Err3 in mice or alternative gene silencing approaches in chick. 


\section{Conclusion and Outlook}

My studies for the first time identified a cell fate determinant of functional motor neuronal subtypes by showing that the orphan nuclear receptors Err2 and Err3 act in a redundant manner to promote gamma-motor neuron identity in a subset of motor neurons (Figure 29). It will further be interesting to elucidate the pathways through which Err2 and Err3 mediate overall gamma-motor neuronal fate and specific aspects of gamma-motor neuron properties, such as soma size or axon caliber and gene expression signatures. This includes testing whether the expression of the recently identified gammaor alpha-motor neuron markers Gfra1, 5HT1D, OPN, and NeuN would be altered by forced expression or loss/knockdown of Err2 and Err3. It would further be interesting to test whether the down-regulation of NeuN activity, directly or indirectly by Err2 and Err3 transcriptional activation, would be a prerequisite for the acquisition of gamma-motor neuron characteristics. Since the lack of direct la afferent synaptic input is a hallmark of the gamma-motor neuron identity, it further has to be tested whether Err2/Err3 activity would influence the formation of la synapse onto motor neurons. Moreover, while I found that expressing Err2 or Err3 in chick triggers motor axon projection defects in limb muscle, it will have to be determined in more detail whether this is indeed accompanied by changes in intrafusal versus extrafusal muscle fiber innervation. Finally, the potentially redundant requirement of Err2 and Err3 for gamma-motor neuron specification will have to be addressed using simultaneous conditional inactivation of Err2 and Err3 in mice or alternative gene silencing approaches in chick. All these studies will give us further insights into the mechanisms mediating the specification of gamma-motor 
neurons, the pathways determining their functional properties, and their contribution to the function of the circuits that control movements.

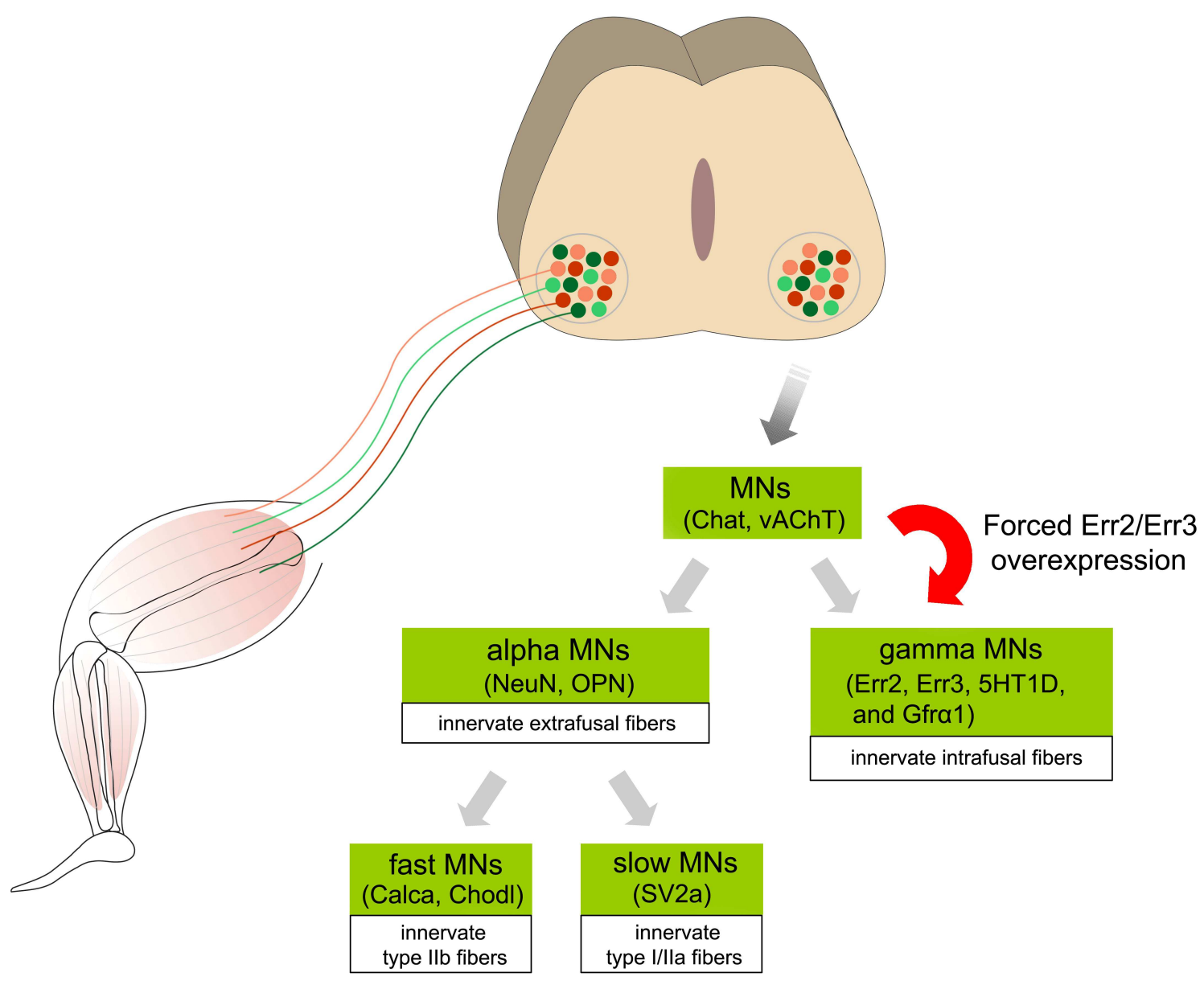

Figure 29. Schematic representation of molecular distinctions of the spinal motor neurons. Motor neurons are divided into alpha or gamma subtypes. Alpha-motor neurons are further subdivided into fast or slow types based on the motor unit they belong to. Chat and vAChT are generic markers for all postmitotic motor neurons. The large-diameter alpha-motor neurons express osteopontin (OPN), high levels of NeuN, and low levels of Err3, while the small-sized gamma-motor neurons express Gfra1, 5HT1d, low levels of NeuN, and high levels of Err2 and Err3. Calca and Chodl are fast motor neurons markers, while SV2A is known to be selectively expressed by slow motor neurons. Forced overexpression of Err2 or Err3 drives a gamma-motor neuronal phenotype. 


\section{References}

Agalliu, D., Takada, S., Agalliu, I., McMahon, A. P. and Jessell, T. M. (2009) 'Motor neurons with axial muscle projections specified by Wnt4/5 signaling', Neuron 61(5): 708-20.

Alaynick, W. A., Kondo, R. P., Xie, W., He, W., Dufour, C. R., Downes, M., Jonker, J. W., Giles, W., Naviaux, R. K., Giguere, V. et al. (2007) 'ERRgamma directs and maintains the transition to oxidative metabolism in the postnatal heart', Cell Metab 6(1): 13-24.

Arber, S., Han, B., Mendelsohn, M., Smith, M., Jessell, T. M. and Sockanathan, S. (1999) 'Requirement for the homeobox gene Hb9 in the consolidation of motor neuron identity', Neuron 23(4): 659-74.

Arber, S., Ladle, D. R., Lin, J. H., Frank, E. and Jessell, T. M. (2000) 'ETS gene Er81 controls the formation of functional connections between group la sensory afferents and motor neurons', Cell 101(5): 485-98.

Banker, B. Q. and Girvin, J. P. (1971) 'The ultrastructural features of the mammalian muscle spindle', J Neuropathol Exp Neurol 30(2): 155-95.

Bennett, D. J. (1994) 'Stretch reflex responses in the human elbow joint during a voluntary movement', J Physiol 474(2): 339-51.

Bessou, P., Emonet-Denand, F. and Laporte, Y. (1963) '[Relation between the conduction rate of motor nerve fibers and the contraction time of their motor units]', C R Hebd Seances Acad Sci 256: 5625-7.

Botterman, B. R. and Eldred, E. (1982) 'Static stretch sensitivity of la and II afferents in the cat's gastrocnemius', Pflugers Arch 395(3): 204-11.

Briscoe, J. and Ericson, J. (1999) 'The specification of neuronal identity by graded Sonic Hedgehog signalling', Semin Cell Dev Biol 10(3): 353-62.

Briscoe, J., Sussel, L., Serup, P., Hartigan-O'Connor, D., Jessell, T. M., Rubenstein, J. L. and Ericson, J. (1999) 'Homeobox gene Nkx2.2 and 
specification of neuronal identity by graded Sonic hedgehog signalling', Nature 398(6728): 622-7.

Brownstone, R. M. and Stuart, D. G. (2011) 'Whither motoneurons?', Brain Res 1409: 93-103.

Burke, R. E., Levine, D. N., Tsairis, P. and Zajac, F. E., 3rd (1973) 'Physiological types and histochemical profiles in motor units of the cat gastrocnemius', J Physiol 234(3): 723-48.

Burke, R. E., Strick, P. L., Kanda, K., Kim, C. C. and Walmsley, B. (1977) 'Anatomy of medial gastrocnemius and soleus motor nuclei in cat spinal cord', J Neurophysiol 40(3): 667-80.

Caubit, X., Thoby-Brisson, M., Voituron, N., Filippi, P., Bevengut, M., Faralli, H., Zanella, S., Fortin, G., Hilaire, G. and Fasano, L. (2010) 'Teashirt 3 regulates development of neurons involved in both respiratory rhythm and airflow control', J Neurosci 30(28): 9465-76.

Chakkalakal, J. V., Nishimune, H., Ruas, J. L., Spiegelman, B. M. and Sanes, J. R. (2010) 'Retrograde influence of muscle fibers on their innervation revealed by a novel marker for slow motoneurons', Development 137(20): 3489-99.

Chalmers, G. (2004) 'Re-examination of the possible role of Golgi tendon organ and muscle spindle reflexes in proprioceptive neuromuscular facilitation muscle stretching', Sports Biomech 3(1): 159-83.

Chen, H. H., Hippenmeyer, S., Arber, S. and Frank, E. (2003) 'Development of the monosynaptic stretch reflex circuit', Curr Opin Neurobiol 13(1): 96-102.

Chen, J. and Nathans, J. (2007) 'Estrogen-related receptor beta/NR3B2 controls epithelial cell fate and endolymph production by the stria vascularis', Dev Cell 13(3): 325-37.

Choe, A., Phun, H. Q., Tieu, D. D., Hu, Y. H. and Carpenter, E. M. (2006) 'Expression patterns of Hox10 paralogous genes during lumbar spinal cord development', Gene Expr Patterns 6(7): 730-7. 
Clarac, F., Cattaert, D. and Le Ray, D. (2000) 'Central control components of a 'simple' stretch reflex', Trends Neurosci 23(5): 199-208.

Coers, C. (1959) 'Structural organization of the motor nerve endings in mammalian muscle spindles and other striated muscle fibers', Am J Phys Med 38: 166-75.

Dalla Torre di Sanguinetto, S. A., Dasen, J. S. and Arber, S. (2008) 'Transcriptional mechanisms controlling motor neuron diversity and connectivity', Curr Opin Neurobiol 18(1): 36-43.

Dasen, J. S., De Camilli, A., Wang, B., Tucker, P. W. and Jessell, T. M. (2008) 'Hox repertoires for motor neuron diversity and connectivity gated by a single accessory factor, FoxP1', Cell 134(2): 304-16.

Dasen, J. S. and Jessell, T. M. (2009) 'Hox networks and the origins of motor neuron diversity', Curr Top Dev Biol 88: 169-200.

Dasen, J. S., Liu, J. P. and Jessell, T. M. (2003) 'Motor neuron columnar fate imposed by sequential phases of Hox-c activity', Nature 425(6961): 926-33.

Dessaud, E., McMahon, A. P. and Briscoe, J. (2008) 'Pattern formation in the vertebrate neural tube: a sonic hedgehog morphogen-regulated transcriptional network', Development 135(15): 2489-503.

Dessaud, E., Yang, L. L., Hill, K., Cox, B., Ulloa, F., Ribeiro, A., Mynett, A., Novitch, B. G. and Briscoe, J. (2007) 'Interpretation of the sonic hedgehog morphogen gradient by a temporal adaptation mechanism', Nature 450(7170): 717-20.

Dufour, C. R., Wilson, B. J., Huss, J. M., Kelly, D. P., Alaynick, W. A., Downes, M., Evans, R. M., Blanchette, M. and Giguere, V. (2007) 'Genome-wide orchestration of cardiac functions by the orphan nuclear receptors ERRalpha and gamma', Cell Metab 5(5): 345-56.

Eccles, J. C., Eccles, R. M., Iggo, A. and Lundberg, A. (1960) 'Electrophysiological studies on gamma motoneurones', Acta Physiol Scand 50: 32-40. 
Eccles, J. C., Eccles, R. M. and Lundberg, A. (1957) 'Durations of after-hyperpolarization of motoneurones supplying fast and slow muscles', Nature 179(4565): 866-8.

Echelard, Y., Epstein, D. J., St-Jacques, B., Shen, L., Mohler, J., McMahon, J. A. and McMahon, A. P. (1993) 'Sonic hedgehog, a member of a family of putative signaling molecules, is implicated in the regulation of CNS polarity', Cell 75(7): 1417-30.

Enjin, A., Leao, K. E., Mikulovic, S., Le Merre, P., Tourtellotte, W. G. and Kullander, K. (2012) 'Sensorimotor function is modulated by the serotonin receptor $1 \mathrm{~d}$, a novel marker for gamma motor neurons', Mol Cell Neurosci 49(3): 322-32.

Enjin, A., Rabe, N., Nakanishi, S. T., Vallstedt, A., Gezelius, H., Memic, F., Lind, M., Hjalt, T., Tourtellotte, W. G., Bruder, C. et al. (2010) 'Identification of novel spinal cholinergic genetic subtypes disclose Chodl and Pitx2 as markers for fast motor neurons and partition cells', J Comp Neurol 518(12): 2284-304.

Eudy, J. D., Yao, S., Weston, M. D., Ma-Edmonds, M., Talmadge, C. B., Cheng, J. J., Kimberling, W. J. and Sumegi, J. (1998) 'Isolation of a gene encoding a novel member of the nuclear receptor superfamily from the critical region of Usher syndrome type lla at 1q41', Genomics 50(3): 382-4.

Friese, A., Kaltschmidt, J. A., Ladle, D. R., Sigrist, M., Jessell, T. M. and Arber, S. (2009) 'Gamma and alpha motor neurons distinguished by expression of transcription factor Err3', Proc Natl Acad Sci U S A 106(32): 13588-93.

Gardiner, P. F. (1993) 'Physiological properties of motoneurons innervating different muscle unit types in rat gastrocnemius', J Neurophysiol 69(4): 1160-70.

Genc, B., Ozdinler, P. H., Mendoza, A. E. and Erzurumlu, R. S. (2004) 'A chemoattractant role for NT-3 in proprioceptive axon guidance', PLOS Biol 2(12): e403. 
Germain, P., Staels, B., Dacquet, C., Spedding, M. and Laudet, V. (2006) 'Overview of nomenclature of nuclear receptors', Pharmacol Rev 58(4): 685-704.

Giguere, V. (1999) 'Orphan nuclear receptors: from gene to function', Endocr Rev 20(5): 689-725.

Giguere, V., Yang, N., Segui, P. and Evans, R. M. (1988) 'Identification of a new class of steroid hormone receptors', Nature 331(6151): 91-4.

Goulding, M. (2009) 'Circuits controlling vertebrate locomotion: moving in a new direction', Nat Rev Neurosci 10(7): 507-18.

Grossmann, K. S., Giraudin, A., Britz, O., Zhang, J. and Goulding, M. (2010) 'Genetic dissection of rhythmic motor networks in mice', Prog Brain Res 187: 19-37.

Hagbarth, K. E., Kunesch, E. J., Nordin, M., Schmidt, R. and Wallin, E. U. (1986) 'Gamma loop contributing to maximal voluntary contractions in man', $J$ Physiol 380: 575-91.

Herrick, C. J. (1947) 'The proprioceptive nervous system', J Nerv Ment Dis 106(3): 355-8.

Hippenmeyer, S., Huber, R. M., Ladle, D. R., Murphy, K. and Arber, S. (2007) 'ETS transcription factor Erm controls subsynaptic gene expression in skeletal muscles', Neuron 55(5): 726-40.

Hippenmeyer, S., Shneider, N. A., Birchmeier, C., Burden, S. J., Jessell, T. M. and Arber, S. (2002) 'A role for neuregulin1 signaling in muscle spindle differentiation', Neuron 36(6): 1035-49.

Hsieh, J. and Gage, F. H. (2005) 'Chromatin remodeling in neural development and plasticity', Curr Opin Cell Biol 17(6): 664-71.

Jessell, T. M. (2000) 'Neuronal specification in the spinal cord: inductive signals and transcriptional codes', Nat Rev Genet 1(1): 20-9. 
Kandel, E. R., Schwartz, J. H. and Jessell, T. M. (2000) Principles of neural science, New York, NY [u.a.]: McGraw-Hill.

Kanning, K. C., Kaplan, A. and Henderson, C. E. (2010) 'Motor neuron diversity in development and disease', Annu Rev Neurosci 33: 409-40.

Katz, B. (1950) 'Depolarization of sensory terminals and the initiation of impulses in the muscle spindle', J Physiol 111(3-4): 261-82.

Kawakami, K. (2007) 'Tol2: a versatile gene transfer vector in vertebrates', Genome Biol 8 Suppl 1: S7.

Kernell, D. (2003) 'Principles of force gradation in skeletal muscles', Neural Plast 10(1-2): 69-76.

Kernell, D., Bakels, R. and Copray, J. C. (1999) 'Discharge properties of motoneurones: how are they matched to the properties and use of their muscle units?', J Physiol Paris 93(1-2): 87-96.

Kuffler, S. W., Hunt, C. C. and Quilliam, J. P. (1951) 'Function of medullated small-nerve fibers in mammalian ventral roots; efferent muscle spindle innervation', J Neurophysiol 14(1): 29-54.

Lee, K. J. and Jessell, T. M. (1999) 'The specification of dorsal cell fates in the vertebrate central nervous system', Annu Rev Neurosci 22: 261-94.

Liem, K. F., Jr., Tremml, G., Roelink, H. and Jessell, T. M. (1995) 'Dorsal differentiation of neural plate cells induced by BMP-mediated signals from epidermal ectoderm', Cell 82(6): 969-79.

Lin, J. H., Saito, T., Anderson, D. J., Lance-Jones, C., Jessell, T. M. and Arber, S. (1998) 'Functionally related motor neuron pool and muscle sensory afferent subtypes defined by coordinate ETS gene expression', Cell 95(3): 393-407.

Lind, D., Franken, S., Kappler, J., Jankowski, J. and Schilling, K. (2005) 'Characterization of the neuronal marker NeuN as a multiply phosphorylated antigen with discrete subcellular localization', J Neurosci Res 79(3): 295-302. 
Lumsden, A. (1995) 'Neural development. A 'LIM code' for motor neurons?', Curr Biol 5(5): 491-5.

Lund, J. P., Richmond, F. J., Touloumis, C., Patry, Y. and Lamarre, Y. (1978) 'The distribution of Golgi tendon organs and muscle spindles in masseter and temporalis muscles of the cat', Neuroscience 3(2): 259-70.

Lund, S. and Pompeiano, O. (1968) 'Monosynaptic excitation of alpha motoneurones from supraspinal structures in the cat', Acta Physiol Scand 73(1): 1-21.

Lundberg, A., Malmgren, K. and Schomburg, E. D. (1987) 'Reflex pathways from group II muscle afferents. 3. Secondary spindle afferents and the FRA: a new hypothesis', Exp Brain Res 65(2): 294-306.

Luo, J., Sladek, R., Bader, J. A., Matthyssen, A., Rossant, J. and Giguere, V. (1997) 'Placental abnormalities in mouse embryos lacking the orphan nuclear receptor ERR-beta', Nature 388(6644): 778-82.

Luo, J., Sladek, R., Carrier, J., Bader, J. A., Richard, D. and Giguere, V. (2003) 'Reduced fat mass in mice lacking orphan nuclear receptor estrogen-related receptor alpha', Mol Cell Biol 23(22): 7947-56.

Mangelsdorf, D. J., Thummel, C., Beato, M., Herrlich, P., Schutz, G., Umesono, K., Blumberg, B., Kastner, P., Mark, M., Chambon, P. et al. (1995) 'The nuclear receptor superfamily: the second decade', Cell 83(6): 835-9.

Manuel, M. and Zytnicki, D. (2011) 'Alpha, beta and gamma motoneurons: functional diversity in the motor system's final pathway', $J$ Integr Neurosci 10(3): 243-76.

McHanwell, S. and Biscoe, T. J. (1981) 'The localization of motoneurons supplying the hindlimb muscles of the mouse', Philos Trans $R$ Soc Lond B Biol Sci 293(1069): 477-508.

McPhedran, A. M., Wuerker, R. B. and Henneman, E. (1965) 'Properties of Motor Units in a Heterogeneous Pale Muscle (M. Gastrocnemius) of the Cat', $J$ Neurophysiol 28: 85-99. 
Mears, S. C. and Frank, E. (1997) 'Formation of specific monosynaptic connections between muscle spindle afferents and motoneurons in the mouse', J Neurosci 17(9): 3128-35.

Mileusnic, M. P. and Loeb, G. E. (2009) 'Force estimation from ensembles of Golgi tendon organs', J Neural Eng 6(3): 036001.

Misawa, H., Hara, M., Tanabe, S., Niikura, M., Moriwaki, Y. and Okuda, T. (2012) 'Osteopontin is an alpha motor neuron marker in the mouse spinal cord', J Neurosci Res 90(4): 732-42.

Mitsunaga, K., Araki, K., Mizusaki, H., Morohashi, K., Haruna, K., Nakagata, N., Giguere, V., Yamamura, K. and Abe, K. (2004) 'Loss of PGC-specific expression of the orphan nuclear receptor ERR-beta results in reduction of germ cell number in mouse embryos', Mech Dev 121(3): 237-46.

Nakamura, H., Katahira, T., Sato, T., Watanabe, Y. and Funahashi, J. (2004) 'Gain- and loss-of-function in chick embryos by electroporation', Mech Dev 121(9): 1137-43.

Novac, N. and Heinzel, T. (2004) 'Nuclear receptors: overview and classification', Curr Drug Targets Inflamm Allergy 3(4): 335-46.

Novitch, B. G., Chen, A. I. and Jessell, T. M. (2001) 'Coordinate regulation of motor neuron subtype identity and pan-neuronal properties by the bHLH repressor Olig2', Neuron 31(5): 773-89.

Oakley, R. A., Lefcort, F. B., Clary, D. O., Reichardt, L. F., Prevette, D., Oppenheim, R. W. and Frank, E. (1997) 'Neurotrophin-3 promotes the differentiation of muscle spindle afferents in the absence of peripheral targets', J Neurosci 17(11): 4262-74.

Onishi, A., Peng, G. H., Poth, E. M., Lee, D. A., Chen, J., Alexis, U., de Melo, J., Chen, S. and Blackshaw, S. (2010) 'The orphan nuclear hormone receptor ERRbeta controls rod photoreceptor survival', Proc Natl Acad Sci U S A 107(25): 11579-84. 
Ovalle, W. K., Jr. (1972) 'Fine structure of rat intrafusal muscle fibers. The equatorial region', J Cell Biol 52(2): 382-96.

Pettersson, K., Svensson, K., Mattsson, R., Carlsson, B., Ohlsson, R. and Berkenstam, A. (1996) 'Expression of a novel member of estrogen response element-binding nuclear receptors is restricted to the early stages of chorion formation during mouse embryogenesis', Mech Dev 54(2): 211-23.

Pfaff, S. L., Mendelsohn, M., Stewart, C. L., Edlund, T. and Jessell, T. M. (1996) 'Requirement for LIM homeobox gene Isl1 in motor neuron generation reveals a motor neuron-dependent step in interneuron differentiation', Cell 84(2): 309-20.

Pun, S., Sigrist, M., Santos, A. F., Ruegg, M. A., Sanes, J. R., Jessell, T. M., Arber, S. and Caroni, P. (2002) 'An intrinsic distinction in neuromuscular junction assembly and maintenance in different skeletal muscles', Neuron 34(3): 357-70.

Roder, L., Vola, C. and Kerridge, S. (1992) 'The role of the teashirt gene in trunk segmental identity in Drosophila', Development 115(4): 1017-33.

Romanes, G. J. (1941) 'Cell columns in the spinal cord of a human foetus of fourteen weeks', J Anat 75(Pt 2): 145-152 1.

Romanes, G. J. (1964) 'The Motor Pools of the Spinal Cord', Prog Brain Res 11: 93-119.

Rousso, D. L., Gaber, Z. B., Wellik, D., Morrisey, E. E. and Novitch, B. G. (2008) 'Coordinated actions of the forkhead protein Foxp1 and Hox proteins in the columnar organization of spinal motor neurons', Neuron 59(2): 226-40.

Sambrook, J. and Russell, D. W. (2001) Molecular cloning : a laboratory manual, Cold Spring Harbor, NY: Cold Spring Harbor Laboratory Press.

Sauer, B. (1987) 'Functional expression of the cre-lox site-specific recombination system in the yeast Saccharomyces cerevisiae', Mol Cell Biol 7(6): 2087-96. 
Schoultz, T. W. and Swett, J. E. (1972) 'The fine structure of the Golgi tendon organ', J Neurocytol 1(1): 1-26.

Shah, V., Drill, E. and Lance-Jones, C. (2004) 'Ectopic expression of Hoxd10 in thoracic spinal segments induces motoneurons with a lumbosacral molecular profile and axon projections to the limb', Dev Dyn 231(1): 43-56.

Sharma, K., Sheng, H. Z., Lettieri, K., Li, H., Karavanov, A., Potter, S., Westphal, H. and Pfaff, S. L. (1998) 'LIM homeodomain factors Lhx3 and Lhx4 assign subtype identities for motor neurons', Cell 95(6): 817-28.

Shirasaki, R. and Pfaff, S. L. (2002) 'Transcriptional codes and the control of neuronal identity', Annu Rev Neurosci 25: 251-81.

Shneider, N. A., Brown, M. N., Smith, C. A., Pickel, J. and Alvarez, F. J. (2009) 'Gamma motor neurons express distinct genetic markers at birth and require muscle spindle-derived GDNF for postnatal survival', Neural Dev 4: 42.

Sladek, R., Beatty, B., Squire, J., Copeland, N. G., Gilbert, D. J., Jenkins, N. A. and Giguere, V. (1997) 'Chromosomal mapping of the human and murine orphan receptors ERRalpha (ESRRA) and ERRbeta (ESRRB) and identification of a novel human ERRalpha-related pseudogene', Genomics 45(2): 320-6.

Smetanin, B. N., Kudinova, M. P. and Shlykov, V. (1982) 'Role of proprioceptive afferentation in the maintenance of equilibrium of an unstable mechanical system', Hum Physiol 8(1): 69-74.

Soukup, T. (1983) 'The number, distribution and size of Golgi tendon organs in developing and adult rat muscles', Physiol Bohemoslov 32(3): 211-24.

Suster, M. L., Sumiyama, K. and Kawakami, K. (2009) 'Transposon-mediated BAC transgenesis in zebrafish and mice', BMC Genomics 10: 477.

Thaler, J., Harrison, K., Sharma, K., Lettieri, K., Kehrl, J. and Pfaff, S. L. (1999) 'Active suppression of interneuron programs within developing motor neurons revealed by analysis of homeodomain factor HB9', Neuron 23(4): 675-87. 
Tourtellotte, W. G., Keller-Peck, C., Milbrandt, J. and Kucera, J. (2001) 'The transcription factor Egr3 modulates sensory axon-myotube interactions during muscle spindle morphogenesis', Dev Biol 232(2): 388-99.

Tremblay, A. M. and Giguere, V. (2007) 'The NR3B subgroup: an ovERRview', Nucl Recept Signal 5: e009.

Uehara, Y. (1973) 'Unique sensory endings in rat muscle spindles', $Z$ Zellforsch Mikrosk Anat 136(4): 511-20.

Vallstedt, A., Muhr, J., Pattyn, A., Pierani, A., Mendelsohn, M., Sander, M., Jessell, T. M. and Ericson, J. (2001) 'Different levels of repressor activity assign redundant and specific roles to $\mathrm{Nkx6}$ genes in motor neuron and interneuron specification', Neuron 31(5): 743-55.

Wada, N. and Shikaki, N. (1999) 'Neuronal pathways for spinal reflexes activated by group I and group II muscle afferents in the spinal segment (Co1) innervating the tail in the low spinalized cat', Exp Brain Res 125(2): 129-38.

Watson, C., Paxinos, G. and Kayalioglu, G. (2008) The Spinal Cord : A Christopher and Dana Reeve Foundation Text and Atlas, [s.I.]: Elsevier professional.

Westbury, D. R. (1982) 'A comparison of the structures of alpha and gamma-spinal motoneurones of the cat', J Physiol 325: 79-91.

Windhorst, U. (2007) 'Muscle proprioceptive feedback and spinal networks', Brain Res Bull 73(4-6): 155-202.

Wine-Lee, L., Ahn, K. J., Richardson, R. D., Mishina, Y., Lyons, K. M. and Crenshaw, E. B., 3rd (2004) 'Signaling through BMP type 1 receptors is required for development of interneuron cell types in the dorsal spinal cord', Development 131(21): 5393-403.

Yu, S., Wang, X., Ng, C. F., Chen, S. and Chan, F. L. (2007) 'ERRgamma suppresses cell proliferation and tumor growth of androgen-sensitive and androgen-insensitive prostate cancer cells and its implication as a therapeutic target for prostate cancer', Cancer Res 67(10): 4904-14. 
Zelena, J. (1976) 'The role of sensory innervation in the development of mechanoreceptors', Prog Brain Res 43: 59-64.

Zhang, X., Zhang, J., Wang, T., Esteban, M. A. and Pei, D. (2008) 'Esrrb activates Oct4 transcription and sustains self-renewal and pluripotency in embryonic stem cells', J Biol Chem 283(51): 35825-33.

Zhou, Q. and Anderson, D. J. (2002) 'The bHLH transcription factors OLIG2 and OLIG1 couple neuronal and glial subtype specification', Cell 109(1): 61-73. 


\section{Curriculum Vitae}

\section{BASIC INFORMATION}

Name: Tsung-I Lee

Nationality: Taiwan

Date of Birth: 12 Aug 1982

Email: Veltine@gmail.com

\section{EDUCATION}

2000-2004

B.S., Department of Biology, Tunghai University

2004-2006

M.S., Institute of Life Sciences, National Cheng Kung University

2008-present European Neuroscience Institute, Göttingen

Laboratory of Developmental Neurobiology

\section{PRESENTATION-POSTER}

Lee, T.I., Müller D., Marquardt T., 2010. Orphan nuclear receptors regulate motor neuron development. Soc. Neurosci. 